\title{
Antibacterial textiles
}

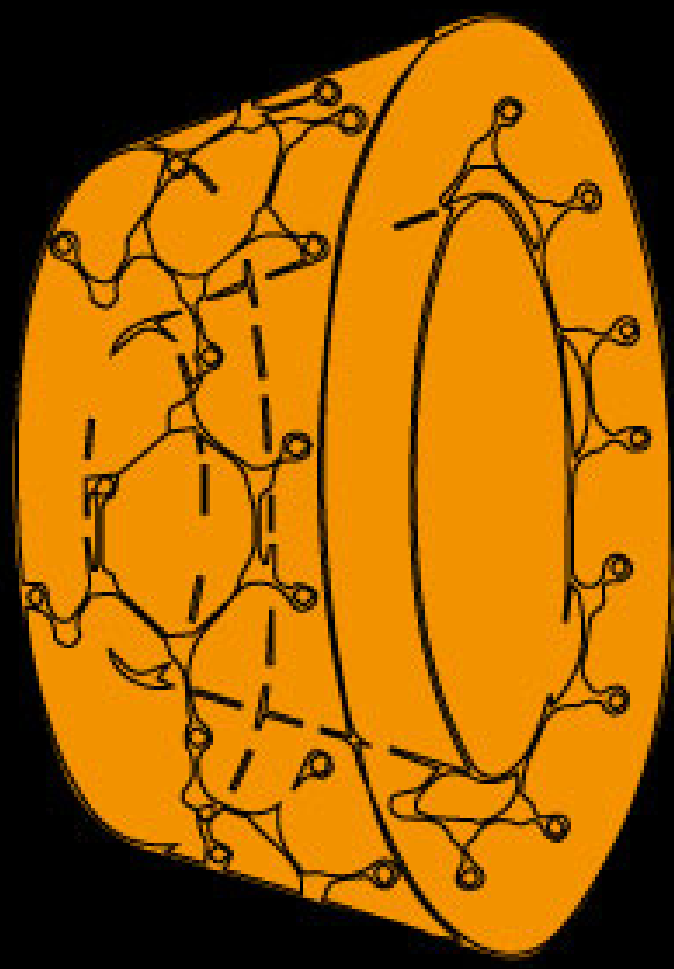

Usha Rashmi Bhaskara 
ANTIBACTERIAL TEXTILES 


\section{DISSERTATION COMMITTEE}

Chairman: Prof. Dr. G.P.M.R. Dewulf, University of Twente, The Netherlands

Promotor: Prof. Dr. Ir. M.M.C.G. Warmoeskerken, University of Twente, The Netherlands

Members:

Prof. Dr. Ir. R. Akkerman, University of Twente, The Netherlands

Dr. R. Hendrix, CERTE, The Netherlands

Prof. Dr. D. Jocic, University of Belgrade, Serbia,

Prof. Dr. Ir. V. A. Nierstrasz, University of Boras, Sweden

Dr.Ir. H. Gooijer, University of Twente, The Netherlands

This work has been financially supported by the E.E.C. project Wash and Load (FP7-SME-2011-2-286831)

U. R. Bhaskara

Antibacterial textiles

Thesis, University of Twente, The Netherlands

ISBN 978-90-365-3873-2

Print : Gildeprint, The Netherlands

Cover design: ( $\beta$-cyclodextrin molecule) U.R. Bhaskara

(C) U.R. Bhaskara, Enschede, 2015

No part of this work may be produced by print, photocopy or any other means without permission in writing from the author. 


\section{ANTIBACTERIAL TEXTILES}

\section{DISSERTATION}

to obtain

the degree of doctor at the University of Twente, on the authority of the rector magnificus,

Prof. Dr. H. Brinksma,

on account of the decision of the graduation committee,

to be publicly defended

on Thursday 23 of April 2015 at 12.45 hrs.

by

U. R. Bhaskara

born on $1^{\text {st }}$ April 1978

in Bangalore, India 
Dit proefschrift is goedgekeurd door de promotor Prof. Dr. Ir. M.M.C.G. Warmoeskerken 


\section{Contents}

Chapter 1 General introduction to Antimicrobial textiles .................................................1

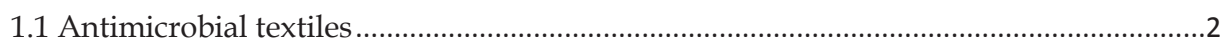

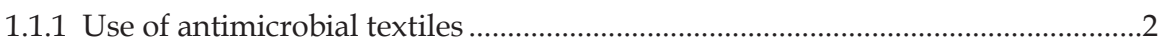

1.1.2. The treatment of textiles with antimicrobial agents ..............................................

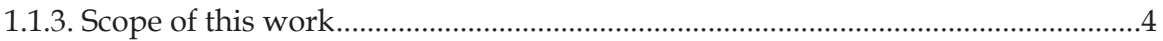

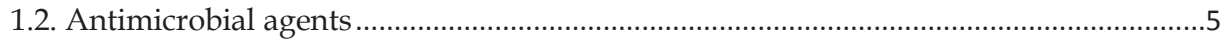

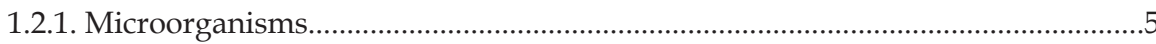

1.2.2. Bacterial microbes on textiles ...........................................................................

1.2.3. An introduction to antimicrobial agents ..............................................................

1.2.4. Antimicrobial agents for textiles..........................................................................

1.2.5. Concerns regarding antimicrobial finishes ........................................................13

1.3. Application of antimicrobial agents in this work ..................................................... 15

1.3.1. Single step and Multi-step method of functionalization of textiles....................15

1.3.2. Selection of antibacterial agents..........................................................................

Chapter 2 Antibacterial textile standards and testing methods .....................................21

2.1. Introduction to antibacterial textile testing methods .................................................. 21

2.1.1. International standards and testing methods .....................................................22

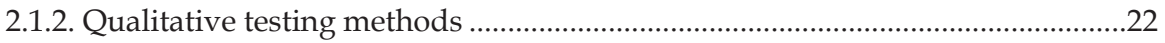

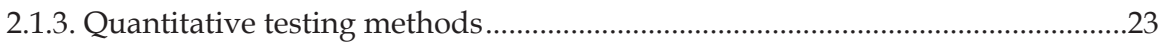

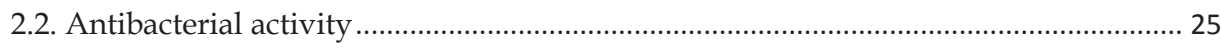

2.3. Selected testing standards and testing methods for this work .................................... 28

Chapter 3 Time survivor study of bacteria on cotton substrate treated with polyhexamethylene biguanide........................................................................................................31

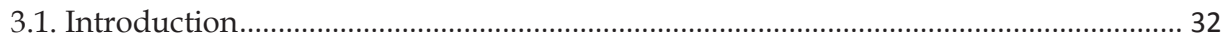

3.1.1. The structure and antibacterial mechanism of polyhexamethylene

biguanide 
3.1.2. Fixation of polyhexamethylene biguanide to cotton ...........................................34

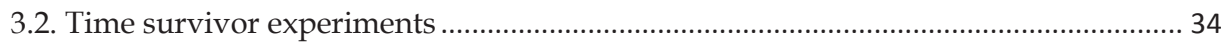

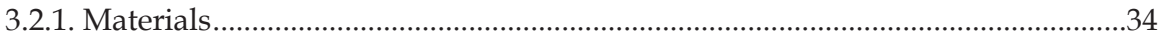

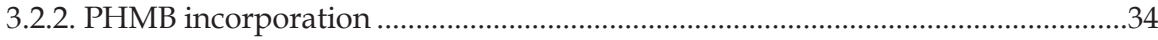

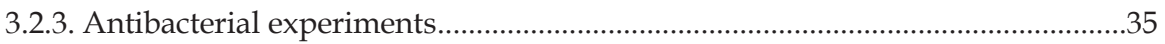

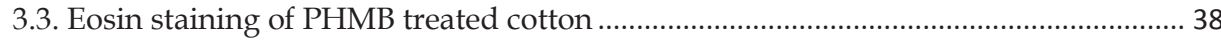

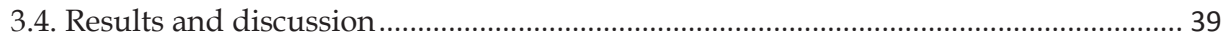

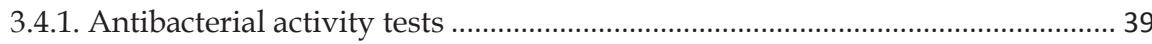

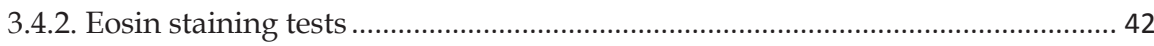

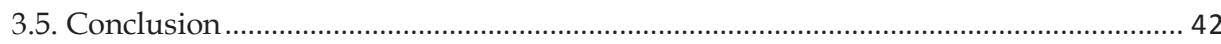

Chapter 4 Single step functionalization of textile substrates .........................................45

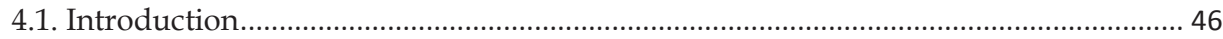

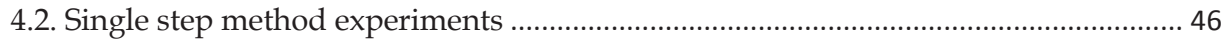

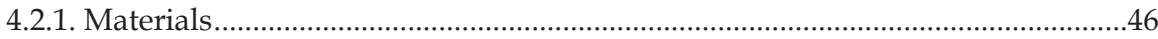

4.2.2. Experimental conditions for the single step method ............................................46

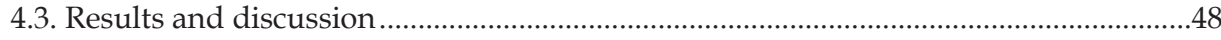

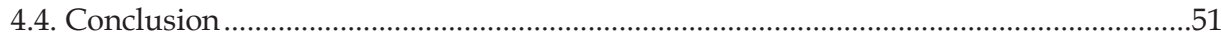

Chapter 5 Textile adsorption and desorption kinetics of PНМВ...............................53

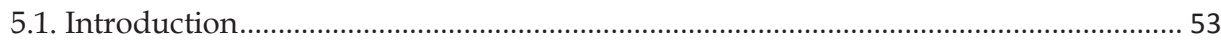

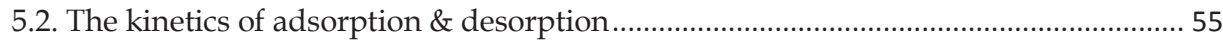

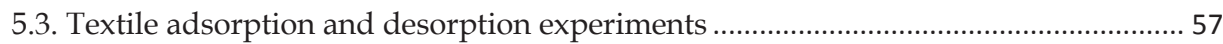

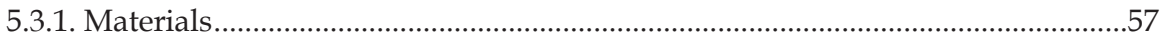

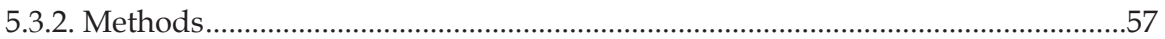

5.3.2.1. The experimental conditions for the adsorption \& desorption

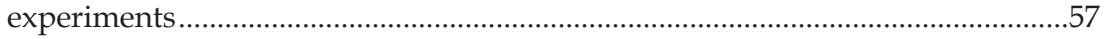

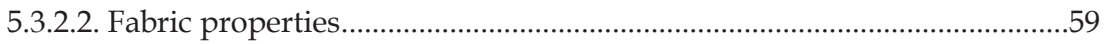

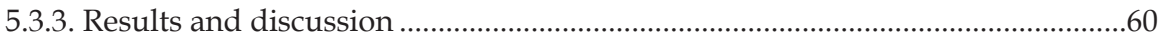


5.3.3.1. The experimental PHMB surface concentrations of cotton, blends \& polyester.

5.3.3.2. The adjusted model and determination of the adsorption rate constants.

5.3.3.3. The model PHMB surface concentrations on cotton \& blends .66

5.4. Conclusion 67

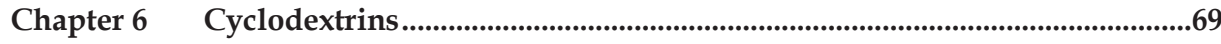

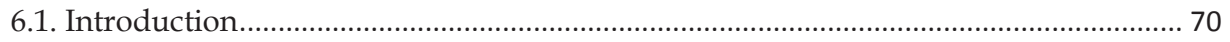

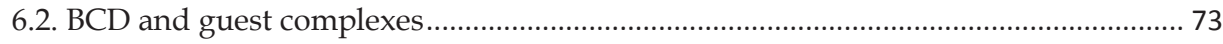

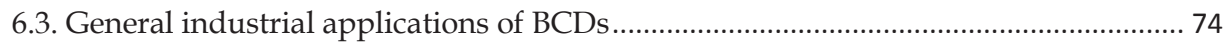

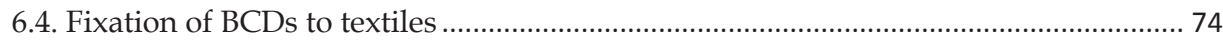

6.5. Quantification and characterization of BCD fixed on textile substrate ....................... 78

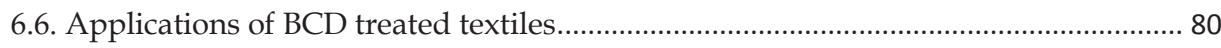

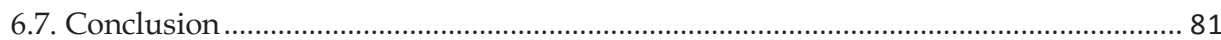

Chapter 7 Attachment of unmodified $\beta$-cyclodextrin and $\beta$-cyclodextrin

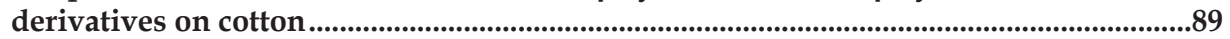

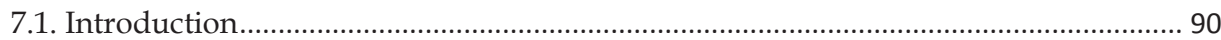

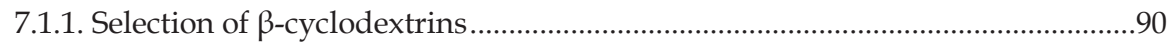

7.1.2. Selection of crosslinker, catalyst and curing conditions.....................................91

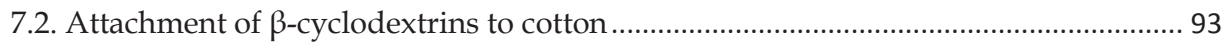

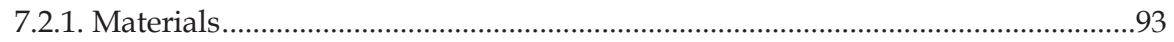

7.2.2. Fixation with the different types of $\beta$-cyclodextrin...........................................93

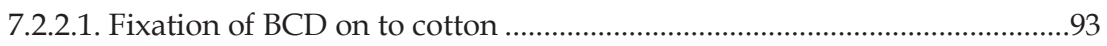

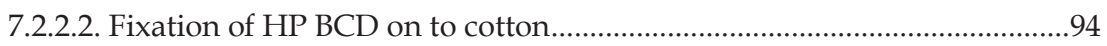

7.2.2.3. Fixation of MCT BCD on to cotton ….........................................................94

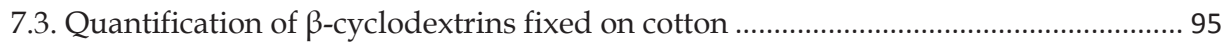

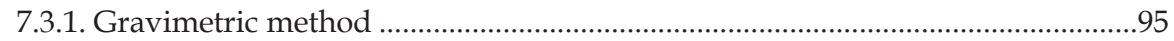


7.3.2. Phenolphthalein method . .95

7.3.3. The influence of BTCA on the Phenolphthalein tests . .97

7.4. Characterization tests of BCD treated textiles 99

7.4.1. FTIR-ATR spectroscopy measurements 100

7.4.1.1. Background 100

7.4.1.2. FTIR analysis of BTCA crosslinked cotton 101

7.4.1.3. FTIR-ATR analysis in this work 102

7.4.2. Differential scanning calorimetry measurements 103

7.5. Laundering tests 104

7.6. Results and discussion 104

7.6.1. Quantification of cyclodextrins fixed on cotton 104

7.6.1.1. Gravimetric measurements .104

7.6.1.2. Phenolphthalein method 106

7.6.2. FTIR-ATR spectroscopy measurements 109

7.6.3. Differential scanning calorimetry measurements 112

7.6.4. Laundering tests 115

7.7. Conclusion .117

Chapter 8 Antimicrobial functionalization of BCD treated fabrics .123

8.1. Introduction 124

8.2. Materials 125

8.3. Activation of polyester. 125

8.3.1. Surface chemical analysis of activated polyester with XPS 126

8.4. Fixation of $\mathrm{BCD}$ to the various textile substrates 127

8.4.1. Quantification of fixed BCD on the various substrates 127

8.4.2. Moisture content measurements of $B C D$ treated samples 128

8.5. UV vis spectrophotometer measurements of $\mathrm{CHXB-BCD}$ and $\mathrm{CHXA-BCD}$ solutions 
8.5.2. UV vis spectrophotometric studies of $\mathrm{CHXB-BCD}$ and $\mathrm{CHXA-BCD}$ solutions

8.6. Treatment of cotton, BCD-cotton, APES and BCD-APES fabrics with CHXA ......... 132

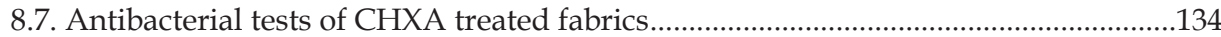

8.8. The release of CHXA from BCD-cotton-CHXA and cotton-CHXA fabrics.................136

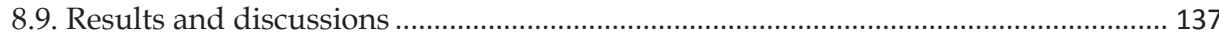

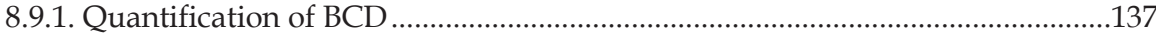

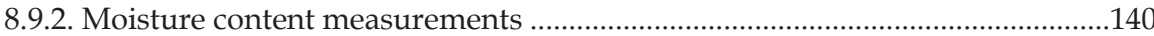

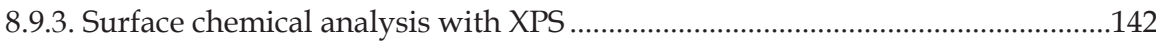

8.9.4. UV vis spectrophotometer measurements of $\mathrm{CHXB-BCD}$ and $\mathrm{CHXA-BCD}$ solutions

8.9.5. Adsorption of CHXA on to cotton, BCD-cotton, APES and BCD-APES fabrics.

8.9.6. Antibacterial experiments of CHXA treated fabrics 152

8.10. Conclusion 156

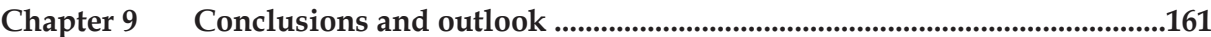

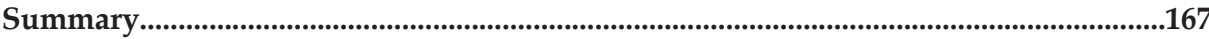

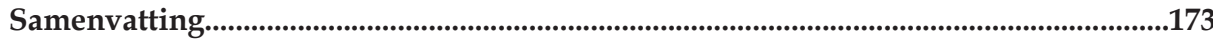

Acknowledgements.........................................................................................................................177

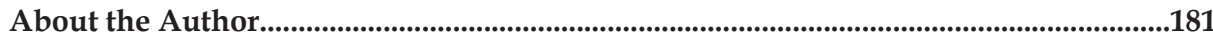



Chapter 1 General introduction to Antimicrobial textiles 


\subsection{Antimicrobial textiles}

Antimicrobial is a term used to describe the action of growth inhibition or destruction of microorganisms [1]. Antimicrobial agents can be physical or chemical agents and they broadly encompass sterilizers, disinfectants, antiseptics, preservatives, sanitizers, biocides, etc. Antimicrobial textiles are textiles that contain antimicrobial agents delivering the antimicrobial action. The antimicrobial agents can be incorporated into the fibres during the fibre production process such as with synthetic polymers or they can be applied onto a fabric by an antimicrobial finishing step.

\subsubsection{Use of antimicrobial textiles}

One of the earliest antimicrobial agents on textiles were metallic salts on uniforms and army tents to make them resistant to rotting by a microbial attack during World War II [2]. In the last decade, there has been an increasing interest in antimicrobial finishes for textiles within the framework of textiles with added functionalities. The purposes of an antimicrobial finish are a) to prevent the degradation of textile as a result of a microbial attack of the fibres b) to prevent malodour produced by microbes and c) to prevent the growth and cross contamination of pathogenic microbes $[3,4]$.

Antimicrobial agents on textiles effectively prevent biodegradation of textiles and prolong consequently the life and appearance of the fabric [5]. Preventing malodour gives a longer feeling of freshness of the fabric. An antibacterial treatment of work wear used in hospitals and the food industry could reduce the risk of cross contamination [6-8].

\begin{tabular}{|c|c|}
\hline Applications & Examples \\
\hline Medical & Wound dressings \\
\hline Military & T shirts, socks, underwear \\
\hline Apparel & Active wear, sportswear, socks, shirts, shoes \\
\hline Industrial & Uniforms, work wear \\
\hline Healthcare & Patient gowns, drapes, bed covers, etc \\
\hline
\end{tabular}

Table 1.1: Applications of antimicrobial finished textiles [10]. 
Antibacterial textiles are also applied in medical treatments of skin disorders or in wound healing [9]. In Table 1.1 lists some examples of the application of antimicrobial textiles.

\subsubsection{The treatment of textiles with antimicrobial agents}

Antimicrobial treatments of fabrics are done with the traditional padding method, spraying, coating or with the treatment bath process. The obtained antimicrobial textiles are classified into two categories depending on type of the attachment of the antimicrobial agent to the textile. These agents can be physically or chemically bound to a textile. When the agents are physically bound, they are called leaching antimicrobial agents. In this case the agent is adsorbed onto the textile by electrostatic interactions or via van der Waals forces and the antimicrobial activity of the agent is attributed to its gradual release from the textile into the surroundings in the presence of moisture [11]. The textiles treated with such agents lose their efficacy in time as the agent concentration on the substrate surface reduces due to the leaching process. The leaching type of agents have poor wash fastness i.e. they are easily removed in the laundry process. The chemically bound agents which are attached through covalent bonding to the textiles show good wash fastness and the antimicrobial agent concentration on substrate surface does not show a reduction in time [11]. The antimicrobial agents in this case kill the microorganisms as they come in contact with the molecule of the agent fixed at the surface of the textile. The mechanisms of leaching and chemically bound antimicrobial agents are schematically shown in Figure 1.1.

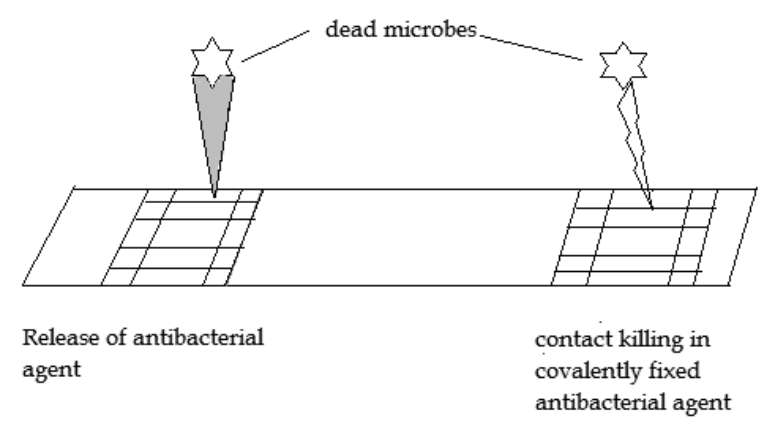

Figure 1.1: Leaching antimicrobial agents versus bound antimicrobial agents [12]. 
This two types of antibacterial materials just described are also classified as active antimicrobial textiles. In case of passive antimicrobial textiles the microorganisms are prevented from attaching themselves to the fibre surface [13]. Oil and water repellent treatments give textiles a passive antimicrobial property. Water repellent chemicals achieve this by reducing the adhesion between the microbes and the cellulose. Such surfaces also do not have the moisture required for the growth of microbes. Modifying the topology of the fibre surface (for instance, Lotus effect) also gives fabrics a passive antimicrobial property.

\subsubsection{Scope of this work}

The theme of this work is the antimicrobial functionalization of textiles and its application in professional laundry companies. Such companies own textile packages which are delivered to the customers such as food industries, hotels and hospitals. After usage, they clean the packages and deliver them again to the customers. The customers from food industry, hotels and hospitals work in an environment in which there is a big chance of contamination and cross-contamination of textiles with bacteria and fungi. In order to reduce the risk of this contamination there is currently a demand for textiles with an antibacterial property. It is possible for the laundry companies to buy commercially available antibacterial textile packages. However, it is known that the level of the antibacterial properties of these kind of fabrics decrease in time, i.e. during the laundry and during the usage. Therefore laundry companies are looking for a method with which they can easily give their textile an antibacterial property which is restored continuously during the washing process. Although the laundry companies pay a lot of attention to the hygiene of textiles, for example through a high temperature bleaching process, the washed fabrics do not have an antimicrobial property.

The laundry process consists of several steps: pre-wash, main wash, bleaching, neutralization, and finally the rinsing step. The most obvious place for adding antimicrobial agents to the fabric is the rinsing step which can be used as an treatment bath. In this way the antibacterial treatment of the fabrics can be done without any effect on the installed base and on the processing conditions. In this way it also allows the usage of antibacterial agents which have no or a very poor washing 
fastness. It is important that the chemicals used to achieve an antimicrobial property of the textiles may not be toxic and may not create allergic reactions to the user. Additional requirements are that the chemicals used are cost effective and that they do not affect the physical properties of the textile like handle, water uptake, drapeability, etc.

\subsection{Antimicrobial agents}

\subsubsection{Microorganisms}

The term 'microorganisms' refers to single celled or multicellular organisms that include bacteria, algae, fungi, viruses, protozoa, etc. They are microscopic in dimensions and can be found almost everywhere. They are essential for life on earth. They are used in various industrial processes such as brewing, cheese making, production of certain chemicals, water treatment, etc. They also contribute to the spoilage of food, biodegradation of materials and in causing disease, etc.

Microorganisms that are frequently found on textiles are bacteria and fungi.

In this work only the bacterial variety of microorganisms are considered. Bacteria have a typical size of a few micrometers and can have different shapes such as rods, spheres, or spirals. The human body carries a large amount of bacteria on its skin and in the gut.

The bacterial structure consists of a cell wall which encloses the inner body, called the cytoplasm. The building components of the cell wall are peptidoglycans and polysaccharides which are crosslinked by peptides. Bacteria can be classified into gram negative and gram positive depending on the differences in bacterial cell wall [16]. The gram positive bacteria possess a thick cell wall with many layers of peptidoglycan and fatty acids. The gram negative bacteria have fewer layers of peptidoglycan and the cytoplasm is surrounded by a lipid membrane and a cytoplasm membrane. This cytoplasm membrane is made of lipopolysaccharides and lipoproteins. The differences in the cell wall between gram positive and gram negative bacteria can be seen in Figure 1.2. The complicated structure of the cell wall of the gram negative bacteria makes it less susceptible to certain antibacterial agents in comparison to the gram positive bacteria. The cytoplasmic membrane separates the cell's internal structure from the outer environment. It regulates the flow of 
molecules in and out of the cytoplasm. The cytoplasm also contains the DNA and plasmids, granules, ribosomes, mesosomes, pili, as shown in the figure below.

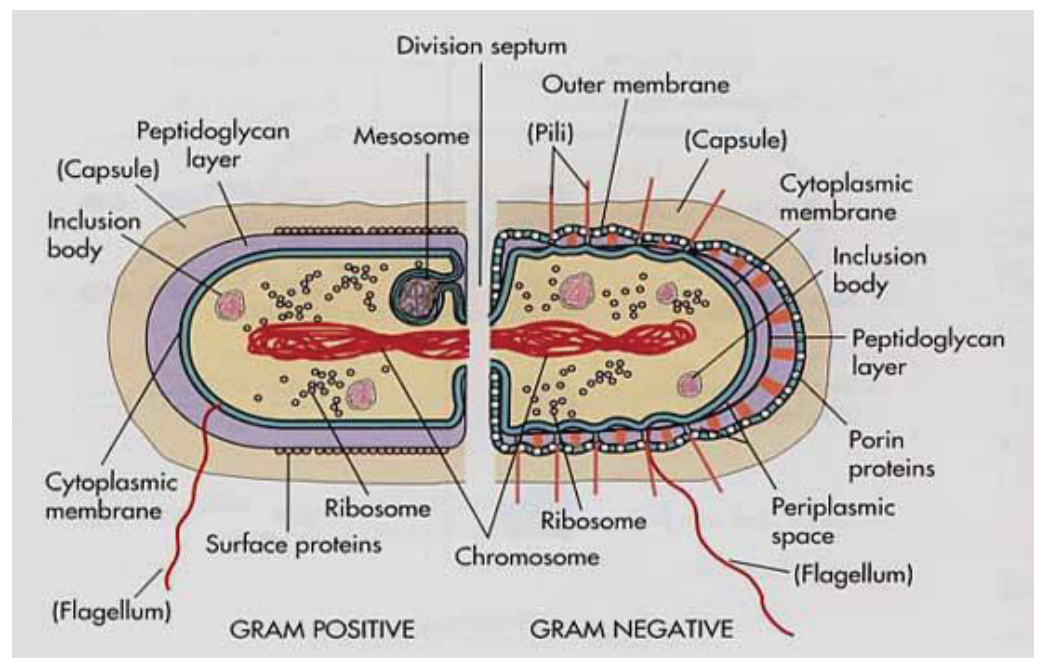

Figure 1.2: Bacteria gram negative and gram positive bacteria [14].

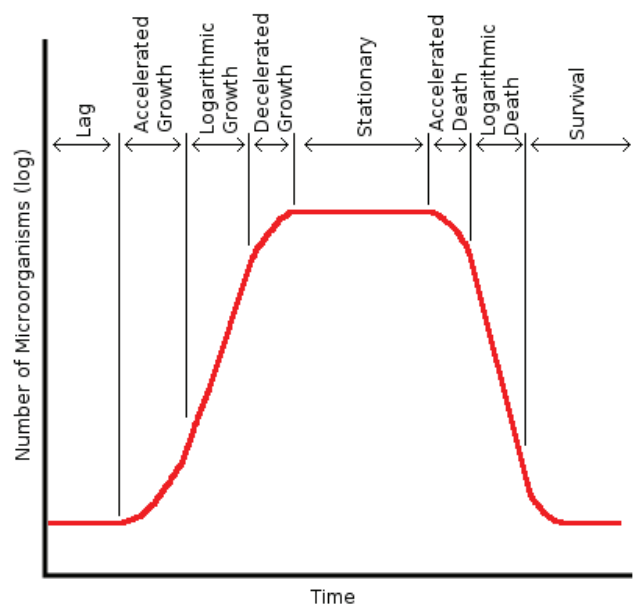

Figure 1.3: Stages of bacterial growth [16].

The bacterial growth in an closed nutrient containing environment shows the following pattern; a lag phase, accelerated growth phase, followed by a log growth phase. As the nutrients start to deplete in the environment, a decelerated growth phase is seen. This is followed by a stationary phase and finally a death phase. This is depicted in Figure 1.3. Bacteria such as Escherichia coli grow rapidly under the right 
conditions, doubling every 15-20 minutes. The right conditions here refer to temperature, $\mathrm{pH}$, moisture, availability of nutrients, etc.

\subsubsection{Bacterial microbes on textiles}

Everyday clothing, carpets, home furnishings, floor coverings, mops, hospital bedding and sheets, etc all carry bacteria of various types. Clothing carry microorganisms on their surface due to the transfer of the bacteria from the wearer's skin. The spectrum of bacteria found on the skin consist of the following strains; Staphylococcus aureus, Coryne bacteria brevi, Proprioni bacteria, micrococcus bacteria, Peptococcus bacteria, Escherichia coli and B. subtilis $[2,18]$. The presence of bacteria leads to malodour, colouring and degradation of textile. Degradation of textiles is very clearly seen in textiles exposed to waterlogged or humid conditions or in microbiologically active soils.

It is known that bacteria adhere to textile surfaces quite well. However, the distribution of the bacteria is not homogenous due to the differences in roughness and texture of the textile. Bacteria adhere more easily to cotton than polyester or blends due to the rougher texture of cotton in comparison to the smoother and the more uniform surface of polyester [15]. The hydrophilic cotton also has a higher moisture content than polyester which is more conducive for the growth of bacteria.

Bacteria are also responsible for the biodeterioration of fibres. The carbon in the molecules of the textile fibre is a food source for microorganisms. Additives on the fabric surface such as sizes, anti-stat chemicals and lubricants are also a food source for bacteria. The bacteria produce enzymes such as endoglucanase, cellobiohydrolase and $\beta$-D-glucosidase. The endoglucanases hydrolyze the $\beta$-glycosidic bonds of cellulose to produce cellooligosaccharides. The cellobiohydrolases release the disaccharide residues from the non-reducing ends of cellulose molecules. The $\beta$-Dglucosidases hydrolyze the disaccharide residues and the soluble cellooligosaccharides to glucose [15]. Due to this hydrolysis, the degree of polymerization of the cellulose chain reduces leading to the reduction in the fibre breaking strength. A similar hydrolysis is seen in microbial attack of synthetic fibres where the bacterial enzymes break down the polymer structures into smaller 
oligomers, dimers and monomers. These smaller molecules can then be transported through the outer membrane of the bacteria.

The biodeterioration of textiles is evident as surface discolouration. The discolouration may be due to some pigmented substance excreted by the microorganism or due to some byproduct of the hydrolysis [18].

Human beings carry with them a variety of harmless and disease carrying pathogenic bacteria. One significant problem regarding the textile being a medium of carrying bacteria is the risk of cross-contamination. Cross-contamination refers to the physical movement of bacteria from one person, object or place to another. The potential for cross-contamination exists all the time everywhere. However, the risk is increased in places such as hospitals where a large of number of people carrying a variety of disease causing pathogens are housed. The so called nosocomial infections acquired in hospitals occur mainly due to the transfer of pathogenic bacteria from contaminated equipment to a patient during diagnostic and therapeutic procedures [20]. It is reported that the hospital floors, furniture, bedding, walls and air filters also carry such pathogenic bacteria and routine disinfection procedures are in place to reduce such bacteria [21]. Pathogenic bacteria have been found on neck ties and gowns of doctors and nurses [26]. Apart from hospitals, such cross-contamination can also occur in hotels, food industries, and slaughter houses. The work wear that is washed in the professional laundries come from these industries.

\subsubsection{An introduction to antimicrobial agents}

The classification of antimicrobial agents can be done on the basis of their activity against specific microorganisms, their killing kinetics, or the mechanism of antimicrobial action. Antimicrobial agents can also be classified as antibacterial, antifungal, sporicidal or virucidal depending on whether the microorganisms they attack are specifically bacteria or fungi, bacterial spores or viruses. Antimicrobial agents can also be broadly classified as either bactericidal/biocidal or bacteriostatic/biostatic. Bactericidal/biocidal agents kill the microorganisms while bacteriostatic/biostatic agents restrict the growth of microorganisms. 
There are several differences between bactericidal and bacteriostatic agents. Bactericidal agents attack many sites within the bacteria while the bacteriostatic agents attack only specific target sites [16]. Bactericidal agents generally rupture the cell membrane and cause irreversible damage to the structure and function of the microorganism. The biocidal injury is mainly related to the cytoplasm membrane [17]. Bacteriostatic agents cause metabolic injury which is reversible after the removal of the antibacterial agent $[18,19]$. The bacteriostatic killing is defined as the killing of $90-99 \%$ of a bacterial load within 18-24 hours while bactericidal killing refers to the reduction of more than $99.9 \%$ of a bacterial load [20]. Since bactericidal agents act on common multiple target sites of various microorganisms they can be effective against a wide array of microorganisms including fungi, spores, viruses [33].

Bactericidal agents can be considered as bacteriostatics when used at higher dilutions [11]. Apart from the agent concentration, the antimicrobial activity of an agent is dependent on several other factors such as the type of the target microorganism, the bacterial numbers, the environmental humidity, temperature, $\mathrm{pH}$ and contact time [21]. The interaction of the agent with an microorganism follows four stages starting with the adsorption of the agent onto the cell surface of the microorganism. This is followed by the interaction of the agent with the outer cell layers of the microorganism. There after an uptake of the agent into the cell occurs and finally the agent attacks the target site within the bacterium as shown in Figure 1.4 [22].

The adsorption isotherms of antimicrobial agents onto the cell surface have been studied by Denyer [19]. He defined several isotherms and reported that the type of isotherm is dependent on the outer composition of the cellular wall of the microorganism, molecular weight of the agent, the hydrophilicity of the agent and the type of microrganism $[22,23]$.

The mechanism of action of different types of antimicrobial agents depends foremost on their chemical composition. Antimicrobial agents are chemically composed of alcohols, aldehydes, bisguanides, bisphenols, halogen releasing compounds, diamidines, heavy metal derivatives, peroxygens, phenols and quaternary ammonium compounds. 


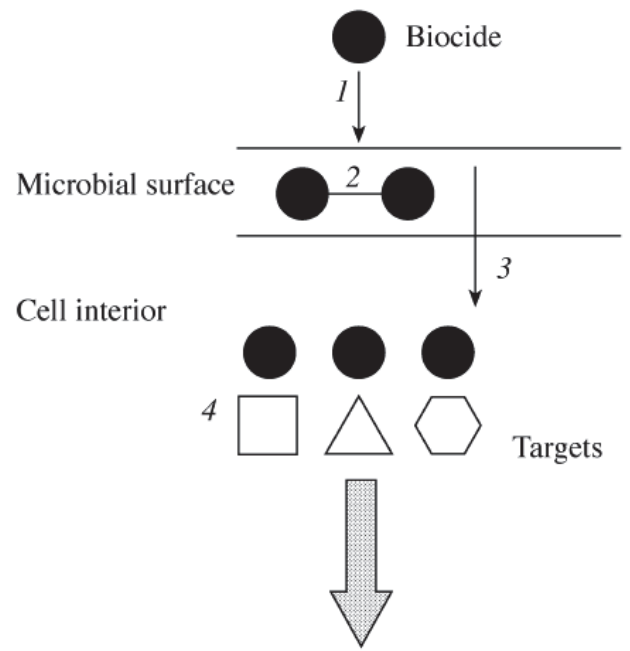

Cell inhibition/inactivation

Figure 1.4: General pattern of antimicrobial agent entry into different microorganisms [22]. 1-adsorption of biocide to the cell surface, 2-interaction with the outer layers, 3-uptake into the cell, 4-interaction with the target site(s).

The attack target site within a bacterium varies depending on the agent composition. For instance, aldehydes based agents target the cell envelope and the proteins of the cell wall while bisguanide based agents attack the phospholipid bilayers cell membrane [24].

\subsubsection{Antimicrobial agents for textiles}

There are several antimicrobial agents that can be used to treat textiles. The application of the agents can be done in a conventional pad-dry method or via an treatment bath method or by other methods such as coating and spraying. Described below are agents that can be chemically finished onto the textile surfaces with any of these methods.

\section{(i). Metals and metals salts}

Metal and metal salts based antimicrobial agents are one of the most commonly used agents for application on textiles [11, 25-27]. These agents work against bacteria by mainly targeting their cell wall. Metal and metal salts also deactivate enzymes in the bacteria by binding with the thiol groups (-SH groups) of the enzymes. Some of the 
mostly commonly used metal based agents are: silver salts, silver based nano particles, copper oxide [28], zinc pyrithione [29], zirconium salts and zeolite complexes [26]. Apart from the application via treatment bath, these agents can also be used as additives in melt extrusion or electroplated onto textile yarns. In order to increase the substantivity of metal salts to textiles, pretreatment of the textiles with certain chemicals can be done. An example of this is the pretreatment of wool with tannic acid which leads to an increase in the number of binding sites for copper and silver ions.

\section{(ii). Quaternary ammonium compounds (QAC)}

Quaternary ammonium compounds are a popular cationic based membrane active agents. These agents target the bacterial cell membrane during their attack. As is the case with membrane active agents the bacterial attack mainly involves the solubilizing of the cell membrane core. These agents are available in a wide range of molecular weights. The chemical composition of these agents is generally $R N R^{\prime} R^{\prime \prime} R^{\prime \prime} X$ where $R, R^{\prime}, R^{\prime \prime}$ and $R^{\prime \prime \prime \prime}$ represent alkyl, alkylaryl, alkoxyl groups while $X$ represents a halide group. Two to three of the $\mathrm{R}$ groupings are simple alkyl groups such as ethyl or methyl group while one is a $\mathrm{C}_{6}$ to $\mathrm{C}_{20}$ hydrocarbon residue [30]. Examples of the low molecular weight QACs are cetrimonium bromide, benzalkonium chloride and cetylpyridinium chloride. It is known that the length of hydrophobic alkyl chain and the number of the ammonium groups of the QAC influence the antimicrobial activity of the agent [11]. Polymeric macromolecules can be made biocidal by incorporating QAC structures into their chain [26]. Examples of such macromolecules are azo and anthraquionone dyes, alginate and chitosan. These agents bind electrostatically with to the anionic cotton via their the cationic groups on their structure. The bulkiness of QAC determines its exhaustion efficiency during the treatment. These agents are deactivated in the presence of anionic detergents.

\section{(iii). Polyhexamethylene biguanides}

The disinfectant polyhexamethylene biguanide is one of the most popular polymeric derivatives from the biguanide family. Biguanides are organic compounds with $\mathrm{HN}\left(\mathrm{C}(\mathrm{NH}) \mathrm{NH}_{2}\right)_{2}$ formula. These are polycationic polymers with a hydrophobic backbone with multiple cationic groups separated by hexamethylene chains. Like the 
QACs, these are membrane active agents. The attack of the bacterium proceeds through the cell membrane core solubilization [19]. This is achieved by attachment of the polyhexamethylene biguanide molecule to the phospholipid heads of cell membrane [31]. The mechanism is further explained in chapter 3. Like with the QACs, polyhexamethylene biguanides can bind to cotton through the electrostatic interaction between the cationic groups on the polyhexamethylene biguanide and the anionic groups on cotton.

\section{(iv). Triclosan}

2, 4, 4'-trichloro-2'-hydroxydiphenyl ether known as Triclosan is a commonly used antibacterial agent in household products such as soaps, toothpastes, cosmetics and deodorants. These agents attack the enzymes responsible for fatty acid synthesis which are required for building the bacterial cell membrane [32].

\section{(v). Chitosan}

Chitosan is a linear polysaccharide composed of randomly distributed $\beta$-(1-4)-linked D-glucosamine and N-acetyl-D-glucosamine. It is obtained through the deacetylation of chitin. Chitin is a component found in shell of crabs, lobsters and scrimps. The positively charged amine groups in its structure destabilize the negatively charged ions on the bacterial cell surface [25]. It is reported that chitosan does not act on any one particular target site within the bacterium. The killing of bacteria is said to be the result of a series of molecular events instigated by the contact of bacteria with chitosan [33]. The molecular weight and the degree of deacetylation of chitosan is known to influence the antimicrobial activity.

\section{(vi). Dyes}

Another unique way of making textiles antimicrobial during other finishing processes is via the dyeing of a textile. One way is to attach an antimicrobial agent to a dye molecule via a cross linker and then dye the textile. Another way is the incorporation of a quaternary ammonium structures into dyes such as mono, diazo and antraquinone dyes [25]. Here the structure of the QAC also influences antimicrobial activity [11]. Some dyes act as antimicrobial agents due to the presence of metal ions such as copper and chromium in their structures. Natural dyes such as 
acacia catechu and quercus infectoria are also known to give effective antimicrobial properties on textiles [34].

\section{(vii). Others}

Addition of organic nitro compounds in wet or dry spinning of nylon, polypropylene, acrylic fibres or polyester can be done to manufacture antimicrobial fibres [35]. Some natural oils such as neem oil, eucalyptus oil can be used to make textiles antimicrobial.

\begin{tabular}{|c|c|c|}
\hline Agent & Primary mode of bacterium & Secondary effects/target site \\
\hline $\begin{array}{l}\text { Silver and other metal/metal } \\
\text { salts }\end{array}$ & $\begin{array}{l}\text { Disruption of protein } \\
\text { synthesis }\end{array}$ & $\begin{array}{l}\text { DNA compression, } \\
\text { metabolic inhibition, cell } \\
\text { membrane } \mathcal{E} \text { cell wall }\end{array}$ \\
\hline QACs & $\begin{array}{l}\text { interaction with } \\
\text { phospholipids bilayer }\end{array}$ & $\begin{array}{l}\text { leakage, cytoplasmic } \\
\text { membrane }\end{array}$ \\
\hline $\begin{array}{l}\text { Polyhexamethylene } \\
\text { biguanide }\end{array}$ & $\begin{array}{l}\text { interaction with } \\
\text { phospholipids bilayer }\end{array}$ & $\begin{array}{l}\text { leakage, cytoplasmic } \\
\text { membrane }\end{array}$ \\
\hline Triclosan & $\begin{array}{l}\text { blocks lipid biosynthesis by } \\
\text { protein enzyme inhibition }\end{array}$ & cytoplasmic membrane \\
\hline Chlorine based & $\begin{array}{c}\text { oxidation of -SH groups \& } \\
\text { disruption of DNA } \\
\text { synthesis }\end{array}$ & $\begin{array}{c}\text { metabolic inhibition, cell } \\
\text { wall/cell membrane }\end{array}$ \\
\hline Chitosan & $\begin{array}{l}\text { membrane disrupter \& } \\
\text { binding with DNA }\end{array}$ & $\begin{array}{c}\text { inhibit mRNA \& protein } \\
\text { synthesis, no single target } \\
\text { site }\end{array}$ \\
\hline Dyes & $\begin{array}{l}\text { Inhibition of nucleic acid } \\
\text { transcription }\end{array}$ & \\
\hline
\end{tabular}

Table 1.2: Table enlisting the primary mechanism of some antibacterial agents [19, $21,36]$.

New possibilities exist with application of antimicrobial peptides and lysozymes onto textile substrates. Table 1.2 summarizes the primary modes and secondary effects of some of the above mentioned antimicrobial agents in their attack against bacteria.

\subsubsection{Concerns regarding antimicrobial finishes}

The antimicrobial treatment of textiles are known to impact the physical and mechanical properties of the textile [37]. A wide spread concern however is the 
impact of these chemicals on the environment and the influence of such finishes on the health of the textile wearer.

A common antibacterial agent used in variety of household consumer products is Triclosan. Triclosan is easily adsorbed into human tissues and has been found in human breast milk and urine. This is a cause for major concern since Triclosan is known to be a endocrine disrupter [38]. Triclosan is often detected in waste water effluent due to the its widespread use in many personal care products. Algae, fish and other aquatic organisms are also known to be sensitive to Triclosan. Triclosan is said to also break down into a dioxin compound which is regarded as an persistent and bioaccumulative pollutant [32]. There is additionally the risk of Triclosan reacting with free chlorine in water bodies or in other consumer products and forming chloroform which has adverse health effects [38].

There is limited research done with regard to influence of antibacterial textiles on the human skin flora. It has been reported that the numbers of skin flora bacteria which is in the order of trillions rejuvenate with time as and when the numbers decrease [39]. It has also been observed that even after repeated applications of a disinfectant on the skin, the numbers of bacteria never reaches zero. It is also been suggested that since the bacteria on skin is distributed three dimensionally, it makes the entry of antimicrobial agents into the different skin layers difficult [2]. The conclusion therefore has been that antibacterial textiles would not disturb the ecological balance of skin flora in a significant way.

In order to increase the survivability in presence of an antimicrobial agent, the microbe start to make changes within its structure. This could be either a change in its cellular structure to impair the agent uptake, through the modification of target site within the bacterium, degradation of the drug inside the bacterium or through the efflux of the antimicrobial agent by the microbe [40]. These just mentioned selfdefense mechanisms are intrinsic to the bacterium but most self-protection mechanisms are acquired by the bacterial cell through mutations or through genes carried on plasmids [21]. Genes carrying plasmids here refers to a small DNA molecules that can be transferred between the bacteria. Due to such mechanisms, the minimum antimicrobial agent concentrations needed to kill certain population of 
bacteria (called as MIC or minimum inhibitory concentration) is not effective after a certain period of time. This eventual emergence of agent resistant bacterial strains leads to ineffective treatments by the agent. This is referred to as antimicrobial resistance of the microorganism. This increased tolerance of microorganisms to an antibacterial agent could lead to increasing tolerance of microorganisms to different classes of antimicrobial agents with similar antimicrobial mechanisms (such as antibiotics). This is referred to as cross resistance [41]. With the widespread use of antimicrobial products there is concern over the risk of antimicrobial resistance of microorganisms and cross resistance to antibiotics [42].

\subsection{Application of antimicrobial agents in this work}

\subsubsection{Single step and Multi-step method of functionalization of textiles}

The aim of this work was the antimicrobial functionalization of textiles. This was to be realized by the application of the chemical during the rinsing step of the laundering cycle. As explained in section 1.1.3, the functional molecule application at every wash cycle would ensure the minimum required agent concentration on the textile surface for the guaranteed required antimicrobial performance. This was to be implemented in professional laundries without altering any of the current process conditions used in the laundering cycle. Therefore all the laboratory experiments done in this work made use of the process conditions prevailing in the rinsing stage of the laundering cycle. Successful results from the laboratory experiments were to be used as a basis for the full scale industrial experiments to be done in a laundry company.

Functional molecules can be applied to textile substrates in two ways. The first method is referred to as the single step method where the chemicals are put into a liquor bath and then a fabric is placed in the bath for certain time. Here the functional molecules fix onto the surface of the fabric depending on the substantivity of the functional molecule to the substrate. This direct application method called as single step method is further elaborated in chapter 4.

The second method is referred to as the multi-step method where the functionalization involves two separate steps. In this method, a host-guest system is used where the host molecule refers to a reservoir molecule which is attached to the 
surface of the textile and the guest molecule refers to the functional molecule that can be held inside the host molecule. These host-guest systems are commonly used for the controlled release of drugs to maximize therapeutic efficiency in the pharmaceutical industry. The host molecules could either have a cavity or could consist of a three dimensional gel structure to enclose the guest molecule. Molecules such as $\beta$-cyclodextrins have a cavity, while hydrogels are three dimensional crosslinked porous gels. The advantage of such a multi-step method is that it allows the treatment of the textiles with functional molecules that show little or no substantivity to the textile. The host molecules further act as a reservoir or storage system enabling the surface of the textile to contain more of the functional molecules on its surface as compared to a fabric without the host molecules.

For this work, $\beta$-cyclodextrins were selected as host molecule. This was mainly motivated by their commercially availability (while hydrogels would have to be synthesized in the laboratory). The biodegradability and non-toxic properties of $\beta$ cyclodextrins also were also considered as advantages. This multi-step method proceeds in two stages: in the first step, $\beta$-cyclodextrins are fixed onto the textile substrates. The application of the guest molecule is done then later in the second step. The work pertaining to the multi-step method is elaborated from chapter 6 to chapter 8 .

\subsubsection{Selection of antibacterial agents}

For the single step method and the multi-step method, a literature review was done to select the suitable antibacterial agents that could be applied. The toxicity information of various chemicals and their applicability to textiles (in the given process conditions) was taken into consideration for the choice of the chemicals.

Polyhexamethylene biguanide was selected as the antibacterial agent for the single step method. This agent is a broad spectrum biocide and is effective against gram positive and gram negative bacteria [43]. It is a polymeric cationic antimicrobial and is a commonly used EPA approved disinfectant in hospitals. It is a membrane active agent and the risk of microbial resistance to this agent is reported to be low. This is elaborated in chapter 3. Polyhexamethylene biguanide is said to be toxic to aquatic fish [44], however it can be safely used in applications where the waste water is not 
dumped into fresh water streams and rivers. It is highly soluble in water allowing an easy application onto textile through a water based liquor bath. It's application on textiles can be done at neutral $\mathrm{pH}$ and the treatment is known to not influence the handle properties in anyway [45]. It is sold as a formulation meant for textile finishing applications (and is also available in a fabric softener product meant for laundries).

For the multi-step process, chlorhexidine diacetate was chosen as the functional molecule. Polyhexamethylene biguanide could not be used for multi-step method. The reasons for this are elaborated in chapter 6 and chapter 8. Chlorhexidine diacetate a cationic bisguanide molecule, is also a widely used disinfectant. It works effectively against both the gram positive and gram negative bacteria. As in the case with polyhexamethylene biguanide, chlorhexidine diacetate is said to be unaffected by the problem with microbe resistance [31]. Finally it has the required molecular weight, hydrophobicity and geometry in order to complex with $\beta$-cyclodextrin [46].

The presented work here is divided into nine parts starting with the introduction to antimicrobial agents and antibacterial textile testing in chapter $1 \& 2$ respectively. Chapter 3 covers a time survivor study of bacteria on polyhexamethylene biguanide treated cotton. It is then followed by the optimization experiments for the single step method of functionalization in chapter 4 . Chapter 5 describes the theoretical model developed to describe the textile adsorption and desorption kinetics of polyhexamethylene biguanide. This chapter includes the experimental work done to validate the model. This work is then followed by chapter $7 \& 8$ which describe the work done in the antibacterial functionalization of textiles with the multi-step method. Chapter 9 summarizes the conclusions of the work done with the single step \& multi-step method and presents recommendations for future investigations.

\section{References}

1. Rouette, H.-K., Encyclopedia of Textile Finishing: CD-ROM. 2000: Springer.

2. Hipler, U. and P. Elsner, Biofunctional textiles and the skin. 2006: S Karger Pub.

3. Hashem, M., et al., Enhancing antimicrobial properties of dyed and finished cotton fabrics. Carbohydrate Polymers, 2009. 78(3): p. 502-510. 
4. Dorugade, V.A. and K. Bhagyashri, Antimicrobial finishing of textiles. ManMade Textiles in India, 2010. 53(3).

5. Raschle, P., Microbial influence on cellulosic textiles and microbiological testing. International Biodeterioration, 1989. 25(1): p. 237-244.

6. Borkow, G. and J. Gabbay, Biocidal textiles can help fight nosocomial infections. Medical hypotheses, 2008. 70(5): p. 990-994.

7. Borkow, G. and J. Gabbay, Preventing Pathogens Proliferation and Reducing Potential Sources of Nosocomial Infections with Biocidal Textiles in Developing Countries. Open Biology Journal, 2010. 3: p. 81-86.

8. Renaud, F.N.R., et al., Evaluation of antibacterial properties of a textile product with antimicrobial finish in a hospital environment. Journal of industrial textiles, 2006. 36(1): p. 89.

9. Ricci, G., et al., Evaluation of the antibacterial activity of a special silk textile in the treatment of atopic dermatitis. Dermatology, 2006. 213(3): p. 224-227.

10. Thiry, M.C., Antimicrobials save the day. AATCC review, 2009. 9(5): p. 21-26.

11. Simoncic, B. and B. Tomsic, Structures of Novel Antimicrobial Agents for TextilesA Review. Textile Research Journal, 2010. 80(16): p. 1721.

12. Tiller, J.C., Antimicrobial surfaces, in Bioactive Surfaces. 2011, Springer. p. 193217.

13. Ramachandran, T., K. Rajendrakumar, and R. Rajendran, Antimicrobial textiles-an Overview. IE (I) Journal-TX, 2004. 84(2): p. 42-47.

14. http://micro.digitalproteus.com/morphology2.php. last accessed date: 5-112014.

15. Bajpai, V., et al., Quantification of bacterial adherence on different textile fabrics. International Biodeterioration \& Biodegradation, 2011. 65(8): p. 1169-1174.

16. Gilbert, P. and A.J. McBain, Potential impact of increased use of biocides in consumer products on prevalence of antibiotic resistance. Clinical Microbiology Reviews, 2003. 16(2): p. 189.

17. Denyer, S. and G. Stewart, Mechanisms of action of disinfectants. International biodeterioration \& biodegradation, 1998. 41(3-4): p. 261-268.

18. Denyer, S., Mechanisms of action of antibacterial biocides. International biodeterioration \& biodegradation, 1995. 36(3-4): p. 227-245.

19. Denyer, S., Mechanisms of action of biocides. International Biodeterioration, 1990. 26(2-4): p. 89-100. 
20. Pankey, G. and L. Sabath, Clinical relevance of bacteriostatic versus bactericidal mechanisms of action in the treatment of Gram-positive bacterial infections. Clinical infectious diseases, 2004. 38(6): p. 864.

21. Russell, A.D., J.R. Furr, and J.Y. Maillard, Microbial susceptibility and resistance to biocides. ASM News-American Society for Microbiology, 1997. 63: p. 481487.

22. Russell, A., Similarities and differences in the responses of microorganisms to biocides. Journal of antimicrobial chemotherapy, 2003. 52(5): p. 750-763.

23. Denyer, S. and J.Y. Maillard, Cellular impermeability and uptake of biocides and antibiotics in Gram-negative bacteria. Journal of applied microbiology, 2002. 92(s1): p. 35S-45S.

24. McDonnell, G. and A.D. Russell, Antiseptics and disinfectants: activity, action, and resistance. Clinical microbiology reviews, 1999. 12(1): p. 147-179.

25. Gao, Y. and R. Cranston, Recent advances in antimicrobial treatments of textiles. Textile Research Journal, 2008. 78(1): p. 60-72.

26. El-Ola, S.A., Recent developments in finishing of synthetic fibers for medical applications. Designed Monomers and Polymers, 2008. 11(6): p. 483-533.

27. Gabbay, J., et al., Copper oxide impregnated textiles with potent biocidal activities. Journal of industrial textiles, 2006. 35(4): p. 323.

28. Nakashima, T., et al., Antibacterial activity of cellulose fabrics modified with metallic salts. Textile Research Journal, 2001. 71(8): p. 688-694.

29. Windler, L., M. Height, and B. Nowack, Comparative evaluation of antimicrobials for textile applications. Environment international, 2013. 53: p. 62-73.

30. Sherrill, J., The evaluation of bacteriostatic reagents and methods of application to textile fabrics. Textile Research Journal, 1956. 26(5): p. 342-350.

31. Gilbert, P. and L. Moore, Cationic antiseptics: diversity of action under a common epithet. Journal of applied microbiology, 2005. 99(4): p. 703-715.

32. Glaser, A., The ubiquitous triclosan. A common antibacterial agent exposed. Pesticides and You, 2004. 24: p. 12-17.

33. Raafat, D., et al., Insights into the mode of action of chitosan as an antibacterial compound. Applied and environmental microbiology, 2008. 74(12): p. 37643773.

34. Singh, R., et al., Antimicrobial activity of some natural dyes. Dyes and Pigments, 2005. 66(2): p. 99-102. 
35. Schindler, W. and P. Hauser, Chemical finishing of textiles. 2004: Woodhead Publishing.

36. Russel, D.A., Similarities and differences in responses of microrganisms to biocides. Journal of antimicrobial chemotherapy, 2003(52): p. 750-763.

37. Cai, Z. and G. Sun, Antimicrobial finishing of acrilan fabrics with cetylpyridinium chloride: affected properties and structures. Journal of applied polymer science, 2005. 97(3): p. 1227-1236.

38. Dann, A.B. and A. Hontela, Triclosan: environmental exposure, toxicity and mechanisms of action. Journal of Applied Toxicology, 2011. 31(4): p. 285.

39. Hoefer, D. and T.R. Hammer, Antimicrobial Active Clothes Display No Adverse Effects on the Ecological Balance of the Healthy Human Skin Microflora. ISRN dermatology, 2011.

40. Russell, A., Antibiotic and biocide resistance in bacteria: introduction. Journal of Applied Microbiology, 2002. 92(s1): p. 1S-3S.

41. Russell, A., Mechanisms of antimicrobial action of antiseptics and disinfectants: an increasingly important area of investigation. Journal of Antimicrobial Chemotherapy, 2002. 49(4): p. 597-599.

42. Hegstad, K., et al., Does the wide use of quaternary ammonium compounds enhance the selection and spread of antimicrobial resistance and thus threaten our health? Microbial Drug Resistance, 2010. 16(2): p. 91-104.

43. Wallace, M.L., Testing the efficacy of Polyhexamethylene Biguanide as Antimicrobial treatment for cotton fabric. AATCC Review, 2001. 1(11): p. 18-20.

44. http://www.epa.gov/oppsrrd1/REDs/phmb_red.pdf. last accessed date: 511-2014.

45. Lonza Group Ltd (Arch chemicals), Reputex 20 technical brief.

46. Qi, H., T. Nishihata, and J.H. Rytting, Study of the interaction between $\beta$ cyclodextrin and chlorhexidine. Pharmaceutical research, 1994. 11(8): p. 12071210. 
Chapter 2 Antibacterial textile standards and testing methods 


\subsection{Introduction to antibacterial textile testing methods}

\subsubsection{International standards and testing methods}

For testing the efficacy of antibacterial textiles, there are several standards developed by national and international standard organizations. Examples of such organizations are the International Organization for Standardization (ISO), American Association of Textile Chemists and Colourists (AATCC), and the Japanese Industrial Standards (JIS). These standards from these different organizations detail the various testing methods in order to measure the antibacterial activity of textiles. The AATCC organization has developed the AATCC 100, AATCC 147 and AATCC 174 standards for testing the antibacterial performance of a textile. The ISO organization has developed the ISO 20743 and ISO 20645 standards. The JIS organization has the JIS L 1902 standard. Some standards describe only one testing method while certain other standards describe two or more methods to test the antibacterial activity.

\subsubsection{Qualitative testing methods}

The antibacterial performance of antibacterial textiles can be tested using qualitative or quantitative methods. In the qualitative method, the antibacterial test indicates if the antibacterial textile shows any antibacterial activity at all. The quantitative methods quantify the exact bacterial killing efficiency of such a treated textile .

The frequently used qualitative test method is the agar diffusion plate test, also called the zone of inhibition test or the halo method. Here an antibacterial fabric is placed on an nutrient agar plate which has previously been inoculated with standard microorganisms such as Staphylococcus aureus and Klebsiella pneumoniae. The nutrient agar plate with the fabric is stored for 18-24 hours in an incubator at a temperature of $37^{\circ} \mathrm{C}$ (standard incubation conditions). As the antibacterial agent leaches from the fabric onto the surface of the agar, a microbe free zone appears and the relative size of this zone is taken as a measure of the antibacterial activity of the fabric. The advantage of this test is that it is visual and not time consuming. The disadvantage is that this test gives only qualitative information and the test is applicable only to the leaching type of antibacterial textiles [1]. This method is unsuitable to quantify the antibacterial activity of a textile. Testing standards based on this testing method are the ISO 20645, AATCC 147, AATCC 174 and SN 195920 testing standards [2]. 


\subsubsection{Quantitative testing methods}

Quantitative test methods are the absorption method, the shake flask method, the transfer method and the printing method [2]. The basic principle of these various testing methods is the quantitative measurement of the amount of bacteria killed by an antibacterial fabric in a certain period of time. The standard microorganisms that are used for these tests are Staphylococcus aureus and Klebsiella pneumoniae.

In the absorption test method, an antibacterial textile is contaminated with a certain volume of bacterial inoculum consisting of a certain number of bacteria. This process is referred to as inoculation. The fabric is then incubated. After the incubation, the bacteria are then extracted from the textile by a rinsing step. The number of living bacteria in the rinsed liquid is then determined by the agar plate method. The number of living bacteria on the textile before and after incubation is expressed in terms of CFUs (colony forming unit). From this difference in the CFUs, the killing efficiency is calculated. The advantage of this test is that it can be used to test fabrics which are treated with leaching or covalently fixed antibacterial agents but it cannot be used for testing of hydrophobic samples due to problems with inoculation (i.e. wetting of the sample with the bacteria). Testing standards that include the absorption test method are JIS L 1902, AATCC 100, ISO 20743 and SN 195924 standards [3-6].

In the transfer test method, an antibacterial textile sample is placed on an nutrient agar plate on which a certain amount of inoculant is already pipetted. The textile sample is pressed onto the surface of the agar plate for a certain period of time under some weight. The sample is then removed and incubated face up in a petri dish. The bacteria on the sample are then extracted with rinsing and the living bacteria are counted with the agar plate method. The advantage of this method is that it is suitable for testing of the antibacterial activity of hydrophobic samples. The disadvantage is that this method is not suitable for testing of yarns. Testing standards based on the transfer method are the ISO 20743 and XP G39 010 [5, 6].

In the shake flask testing method, the antibacterial textile samples are immersed in a bacterial solution inside a flask. The number of living bacteria in this solution is known during the start of the experiment. The flask is mechanically agitated for 
certain period of time and after which the reduction in the number of living bacteria is determined by the agar plate method. This method works well for textiles in which the antibacterial agents are covalently fixed. This method cannot be used for leaching type of antibacterial textiles since the mechanical agitation of the bacterial solution in the flask influences the rate of diffusion of the antibacterial agent from the textile. The advantage of this testing method is that it can be used to test antibacterial yarns and fibers. A testing standard that includes the shake flask method is the ASTM E2149 standard [2].

In the printing method, a bacterial inoculum is poured over a membrane and then filtered to leave only the bacteria over the membrane. These bacteria on the membrane are transferred onto a textile sample using a type of printing system. This printed textile sample is incubated and the bacteria are extracted as described earlier. The agar plating is then done to enumerate the living bacteria. This test method is meant to simulate the transfer of bacteria from dry surfaces. The JIS L 1902 and the ISO 20743 standards describes this testing method [4, 5].

All of the testing methods employ a gram positive and a gram negative type of bacteria. The reason for this is that both these types of bacteria react differently to the different antibacterial agents. The gram negative bacteria differ from gram positive types in their cell wall morphology making them easier to kill with antibacterial agents [7]. All standards suggest the use of Staphylococcus aureus (gram positive bacteria) and Klebsiella pneumoniae (gram negative bacteria) as standard microorganisms. The only exception to this is the Japanese JIS L 1902 standard which recommends specific bacteria depending on the specific end user application of the antibacterial textile. The Japanese standard classifies the end use of the antibacterial textiles into two categories; the deodorant finish textiles and microbial control textiles. The deodorant finish textiles are meant to prevent the growth of bacteria on the textile. The aim of such finishes is to prevent the malodour of the textiles. For these textiles only one test microorganism is recommended: Staphylococcus aureus. The so called microbial control textiles are meant to control the growth of skin bacteria as well as pathogenic bacteria on the textile. The aim of these finishes therefore is to prevent the textile from being a medium for cross-contamination. These textiles can further be classified into textiles for general use or for special use. 
The general use refers to household products while special use refers to products used in medical or equivalent facilities. For the microbial control textiles, the standard test microorganisms are Staphylococcus aureus and Klebsiella pneumoniae. Methicillin resistant Staphylococcus aureus (MRSA) is additionally recommended as a test microorganisms for special use textiles. Escherichia coli and Pseudomonas aeruginosa can be used as optional test microorganisms for the testing of these microbial control textiles.

\subsection{Antibacterial activity}

Many companies selling antibacterial textiles express the antibacterial performance of their products in terms of bacteriostatic, bactericidal, slightly or strongly antibacterial or antibacterial. Apparently there is a lack of well-defined classification of antibacterial textiles. Different companies use different testing standards and testing methods. The killing efficacies of antibacterial textiles tested with different standards are expressed in different ways.

American companies usually work with the AATCC 100 standard, while European companies use the ISO 20743 standard and the Japanese make use of the JIS L 1902 standard. In the AATCC 100 standard the antibacterial activity is expressed as \% reduction of the inoculated bacteria. The antibacterial activity is referred to as the antibacterial activity value in the ISO 20743 standard and as bacteriostatic value in the Japanese JIS 1902 standard. In these standards, the antibacterial performance in expressed in log values.

The \% reduction according to the AATCC 100 standard is given below in Equation 2.1 .

$\%$ Reduction $=\left(C F U_{0}^{*}-C F U_{24}\right) / C F U_{0}^{*} .100$

Where CFU refers to the number of colony forming units of bacteria. The subscripts represent the incubation time, initially $\mathrm{t}=0$ and finally $\mathrm{t}=24$ hours. The superscript * refers to the control samples; the treated sample is without the superscript *.

The bacteriostatic activity value of the Japanese standard or the antibacterial activity value of the ISO standard is given below in Equation 2.2. 
$A A=\left(\log C F U_{24}^{*}-\log C F U_{0}^{*}\right)-\left(\log C F U_{24}-\log C F U_{0}\right)$

or

$A A=\log \left[\frac{C F U_{24}^{*} \cdot C F U_{0}}{C F U_{0}^{*} \cdot C F U_{24}}\right]$

Where AA stands for antibacterial activity.

The CFU, superscript and subscript are the same as described earlier.

The AATCC 100 and the ISO 20743 standards do not specify the minimum antibacterial activity required for a textile to be considered as antibacterial [3]. In the marketing literature of American antibacterial textile products tested according to AATCC 100 standard, a 99\% killing of a bacterial load is considered as acceptable though this is not stated by any regulation. The Japanese standard however states that for a textile to be considered antibacterial, it has to show a minimal antibacterial activity value of 2 .

In order to illustrate the differences in the expressions of the antibacterial performance according to the different standards, antibacterial tests were done on two commercial antibacterial textiles using the absorption test method. The two chosen antibacterial samples are referred to as AG and CW (see Table 2.1. for their properties).

The bacteria used for testing these samples was the Escherichia coli ATCC 11229 (referred to as E coli henceforth). This bacteria was procured from LGC standards [8].

This strain of bacteria is used for testing of disinfectants. $1-3 \times 10^{6} \mathrm{CFU} / \mathrm{ml}$ of inoculant was used to contaminate the textile samples. The tests were done in triplicate. The details of the microbiological testing are explained in chapter 3.

\begin{tabular}{|c|c|c|c|}
\hline Sample & Substrate & Antibacterial agent & $\begin{array}{c}\text { Integration } \\
\text { method }\end{array}$ \\
\hline AG & $\begin{array}{c}\text { Polyester } \\
\text { cotton }\end{array}$ & Polymeric quaternary ammonium \\
compound & padded \\
\hline CW & Cotton & Polymeric agent (not specified) & coating \\
\hline
\end{tabular}

Table 2.1:Properties of the antibacterial textiles. 
The CFUs were determined right after the inoculation at time $=0$ and after incubation at time $=24$ hours for the antibacterial textile samples and their respective control samples. The antibacterial activity value, AA and the \% reduction were then calculated. The CFUs from the inoculated control and treated samples at incubation time of 0 and at incubation time of 24 hours are given below in Table 2.2 in $\log _{10}$ values.

\begin{tabular}{|c|c|c|c|c|c|c|}
\hline Name & $\begin{array}{c}\text { Log } \\
C F U_{0}^{*}\end{array}$ & $\begin{array}{c}\text { Log } \\
C F U_{24}^{*}\end{array}$ & $\begin{array}{c}\text { Log } \\
C F U_{0}\end{array}$ & $\begin{array}{c}\text { Log } \\
C F U_{24}\end{array}$ & AA & \% R \\
\hline AG & 6.5 & 8.7 & 6.5 & 6.7 & 2 & - \\
\hline CW & 6.0 & 8.6 & 6.0 & 0 & 8.6 & $100 \%$ \\
\hline
\end{tabular}

Table 2.2: The results of antibacterial tests on commercial antibacterial fabrics. AA refers to the antibacterial activity value and \% $\mathrm{R}$ refers to \% reduction.

As mentioned earlier according to the JIS L 1902 standard, a sample must show an antibacterial activity value of at least 2 to be considered as antibacterial. As seen in Table 2.2, the sample AG just meets the requirements of the JIS standard. The \% reduction cannot be calculated for sample AG since the CFU numbers on the treated sample at 24 hours exceed the CFU numbers on the treated sample at time 0 .

Therefore sample AG passes the JIS L 1902 standard but fails the AATCC standard.

Due to mathematical reasons the AA formula appears to be more suitable for the calculation of antibacterial activity. It takes into consideration the growth of bacteria on the control sample after 24 hours of incubation time. This allows a range of antibacterial activity to be calculated including the calculation of negative AA values while such calculations are not possible with \% reduction. This is illustrated in the Figure 2.1.

With the AA of the Japanese \& the ISO standard, the bacteriostatic activity value (shown as 1 in Figure 2.1) can be calculated. While with the \% reduction, only the bactericidal activity (shown as 2 in the figure) can be calculated.

The Japanese and ISO standard also specify that for an experiment to be considered valid, the CFUs on the control samples after 24 hours of incubation must be atleast 10 times the original inoculated numbers. 


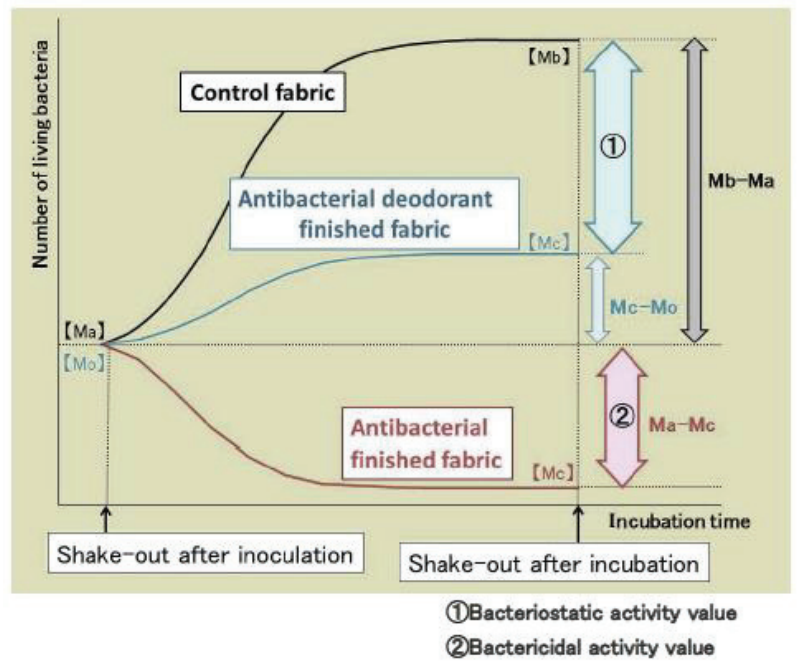

Figure 2.1: The calculation of antibacterial activity according to the Japanese standard. Ma, Mb, Mo \& Mc stand for $\log C F U_{0}^{*}, \log C F U_{24}^{*}, \log C F U_{0}, \log C F U_{24}$ respectively.

This pre-requisite ensures that only the influence of the antibacterial agent on the textile is measured and the influence of nutrients or moisture is excluded during the testing. In conclusion it can be said that due to these reasons the use of the Japanese or the ISO standard is comparatively better than the use of the AATCC standard.

\subsection{Selected testing standards and testing methods for this work}

In this $\mathrm{PhD}$ work the absorption method was selected as the testing method since it can be used to test both hydrophobic and hydrophilic textile samples. Both the ISO 20743 and the JIS L 1902 standards have identical absorption testing methods [9]. However, the Japanese standard is a more comprehensive standard among the three mentioned standards; due to the reason that it offers a possibility to do the testing with microorganisms other than the standard ones and specifically states the required AA limit for a textile to be considered antibacterial. Therefore the Japanese standard was chosen for all the antimicrobial testing done in this work.

During this study, the fabrics functionalized with the single step method were tested in an certified microbiological laboratory with Staphylococcus aureus CCM 4416 and Klebsiella pneumoniae CNCTC 6120 strains according to the Japanese standard. For reasons of costs, an in-house method had been developed based on the JIS L 1902 
absorption method and the E coli ATCC 11229 strain. The samples of antibacterial fabrics obtained via the multi-step approach have been tested with this in-house method. The time-survivor study described in chapter 3 also was conducted using this in-house method. The E coli ATCC 11229 stain is a sturdier strain than that of the Staphylococcus aureus and Klebsiella pneumonia strains used in this work. Therefore antibacterial samples that pass the antibacterial tests with the E coli strain definitely pass the antibacterial tests done with the mentioned two standard strains, however the converse is not the case. In this work, the antibacterial activity for all the antibacterial textiles is calculated from the formula given in Equation 2.2. The minimum antibacterial activity value of 2 as recommended by the Japanese standard has been applied as the threshold value for the treated textiles to be considered antibacterial.

\section{References}

1. Monticello, R.A. and P.D. Askew, Antimicrobial textiles and testing techniques. Russell, Hugo \& Ayliffe's: Principles and Practice of Disinfection, Preservation and Sterilization, 5th Edition, 2013: p. 520-529.

2. Askew, P., Measuring activity in antimicrobial textiles. Chemistry Today, 2009. 27(1): p. 16-20.

3. AATCC Technical manual. 2005, AATCC: USA.

4. JIS (Japanese industrial standard) L 1902:Testing of antibacterial activity and efficacy on textile products. 2002, Japanese Industrial Standard community: Tokyo.

5. ISO 20743: Determination of antibacterial activity of antibacterial finished products. 2007, CEN: London.

6. Teufel, L. and B. Redl, Improved methods for the investigation of the interaction between textiles and microorganisms. Lenzinger Berichte, 2006. 85: p. 54-61.

7. Pinho, E., et al., Antimicrobial activity assessment of textiles: standard methods comparison. Annals of microbiology, 2011. 61(3): p. 493-498.

8. http://www.lgcstandards-atcc.org. last accessed date: 27-10-14.

9. Swofford, H.W., An overview of antimicrobial testing for textile applications. AATCC review, 2010. 6: p. 51-55. 
Chapter 3 Time survivor study of bacteria on cotton substrate treated with polyhexamethylene biguanide 


\subsection{Introduction}

In the field of disinfectants, antibacterial experiments are conducted to study the influence of the disinfectant concentration on the time of disinfection against a selected strain of bacteria. [1, 2]. These studies are called time-survivor or time-kill studies. These kinetic studies provide information that can be helpful in comparing different antibacterial products and their antibacterial activity against different strains of microorganisms.

As stated in chapter 1, polyhexamethylene biguanide was selected as the antibacterial agent for the single step method. The reasons for this choice have been described in section 1.3.4 of chapter 1. This chapter describes the study of the time survivor of bacteria on cotton treated with polyhexamethylene biguanide. The treated fabrics were tested for their antibacterial activity according to the Japanese JISL 1902 standard [3]. In this work, the term 'polyhexamethylene biguanide' refers to the molecule in general, while the acronym PHMB refers to the commercial chemical formulation of polyhexamethylene biguanide used for the treatment of textiles.

\subsubsection{The structure and antibacterial mechanism of polyhexamethylene biguanide}

Polyhexamethylene biguanide is a water soluble polycationic polymer with a hydrophobic backbone having multiple cationic groups separated by hexamethylene chains as shown in Figure 3.1. The terminating end groups can be amine, guanide or cyanoguanide groups.<smiles>[X]C(CC)(CC)CCCNC(=N)NC(=[NH2+])NCCC([Y])(CC)CC</smiles>

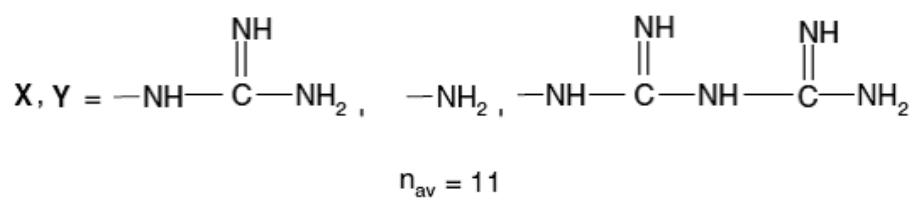

Figure 3.1: Structure of Polyhexamethylene biguanide. Source: reproduced with permission from Elsevier, 2011, [4], Kawabata, A \& Taylor, J 2007. 
Polyhexamethylene biguanide is considered to be a membrane active agent (similar to quaternary ammonium compounds). The bacterial cell membrane is therefore the primary target site of the agent. The antibacterial mechanism starts with the displacement of the divalent cations (such as $\mathrm{Mg}^{2+}$ and $\mathrm{Ca}^{2+}$ ) on the bacterium cell wall as seen in Figure 3.2b. The agent proceeds to interact with the liposaccharides and peptidoglycan layer of cell wall. The polyhexamethylene biguanide molecules then tend to aggregate over the lipid bilayer around the protein sites of the cellular membrane. The proteins gradually lose their function and the membrane slowly starts to solubilize as seen in Figure 3.2c. This finally leads to cellular leakage and loss of membrane permeability barrier as shown in Figure 3.2d [5].

(a)

(b) $\mathrm{Ca}^{++}$

$\mathrm{Ca}^{++}$

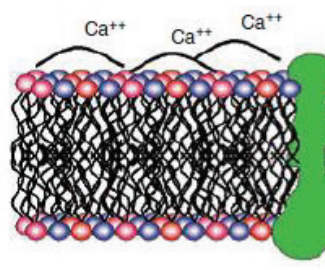

(c)
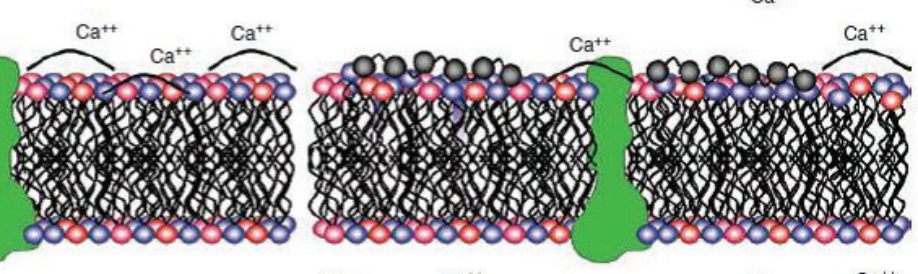

(d)

$\mathrm{Ca}^{++}$

$\mathrm{Ca}^{++}$
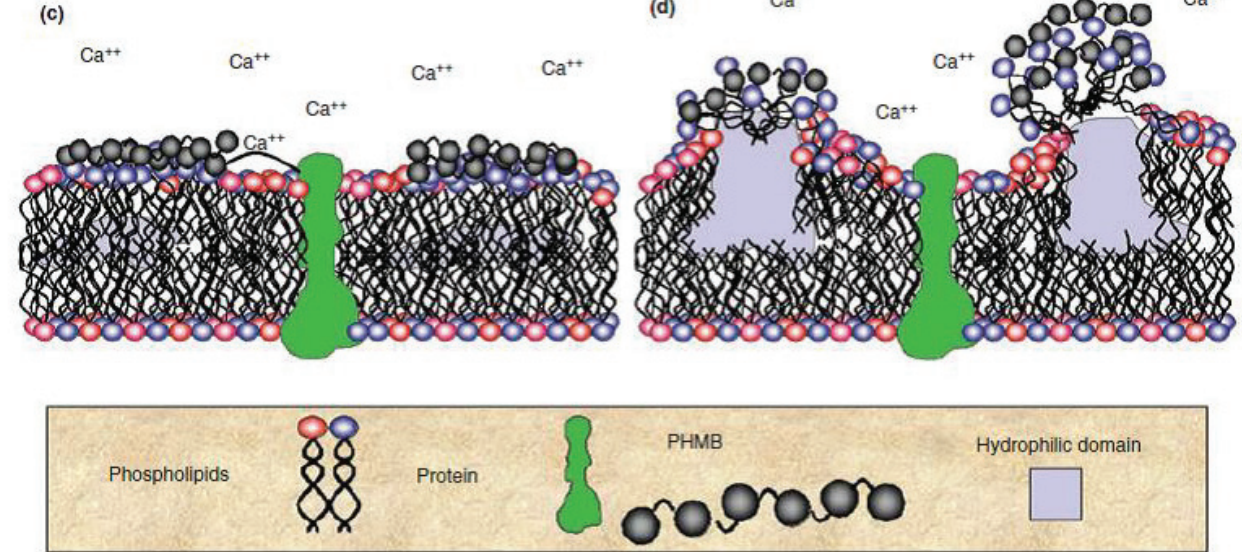

Figure 3.2: Mechanism of antibacterial activity of polyhexamethylene biguanide. Source: reproduced with permission from John Wiley \& Sons, 2011, [5], Gilbert, P \& Moore, L 2005.

The hydrophobic hexamethylene structure in the polyhexamethylene biguanide structure is inflexible and cannot enter the hydrophobic core of cellular membrane. This inability to penetrate is unlike the other membrane active agents such as 
quaternary ammonium compounds. Since the agent does not enter the cell membrane entirely, the bacteria do not have the possibility to adapt to some of the several survival mechanisms such as the efflux mechanism (which is the ejection of the ingested agent by the microbe through specific efflux pumps). This makes this particular agent less susceptible to developing microbe resistance [5].

\subsubsection{Fixation of polyhexamethylene biguanide to cotton}

Cotton can be treated with polyhexamethylene biguanide through the treatment bath method. The cationic biguanide groups on polyhexamethylene biguanide bind to the anionic carboxylate groups on cotton through electrostatic interaction. The adsorption isotherm of this agent onto cotton follows the Langmuir type at lower concentrations and that of Freundlich type at higher concentrations [6].

The bacterial killing efficiency of textiles treated with polyhexamethylene biguanide is widely documented in scientific literature [7-10]. However, none of these reports elucidate the influence of time on the antibacterial activity of such textiles.

\subsection{Time survivor experiments}

\subsubsection{Materials}

A $20 \%$ aqueous polyhexamethylene biguanide hydrochloride stock solution known as Reputex 20 was received from Lonza Group Limited, UK. This procured stock solution contained $0.073 \mathrm{~mol} / 1$ of polyhexamethylene biguanide hydrochloride the molar mass of polyhexamethylene biguanide is $2750 \mathrm{~g} / \mathrm{mol}$ ) [11]. This formulation is henceforth referred to as PHMB. The cotton textile used in this work was bleached white plain woven cotton with a fabric density of $180 \mathrm{~g} / \mathrm{m}^{2}$. The testing microorganism was Escherichia coli ATCC 11229 strain of bacteria obtained from the LCG standard company [12]. The material for the Luria Broth medium and microbiological agar, Triton X100 and Eosin Y (C.I. Acid Red 87, 2' , 4' , 5' , 7' tetrabromofluorescein) were bought from Sigma Aldrich.

\subsubsection{PHMB incorporation}

Textile samples of $30 \mathrm{~cm}$ by $30 \mathrm{~cm}$ were treated with PHMB solutions in an treatment or liquor bath at a temperature of $40^{\circ} \mathrm{C}$ and at $\mathrm{pH} 7$ for $30 \mathrm{~min}$. The $\mathrm{pH}$ was adjusted by the addition of sodium hydroxide or acetic acid drops to the liquor. The liquor to 
cloth ratio (LCR) was 20:1. The amount of PHMB added into the liquor was calculated on the dry weight of the sample and the desired \% o.w.f (on the weight of fabric) as recommended by the supplier of the chemical. The samples were treated with PHMB solutions to obtain $0.4 \%, 0.8 \%, 1.2 \%, 1.6 \%$, and $2.0 \%$ with regard to o.w.f. The corresponding concentrations in the liquor were; $0.2 \mathrm{~g} / 1,0.4 \mathrm{~g} / \mathrm{l}, 0.6 \mathrm{~g} / \mathrm{l}, 0.8 \mathrm{~g} / 1$ and $1.0 \mathrm{~g} / 1$. These application concentrations were that of the formulation Reputex 20 (or PHMB) and not of $100 \%$ polyhexamethylene biguanide solution. After the treatment, the samples were rinsed in cold water once and then air dried.

\subsubsection{Antibacterial experiments}

The antibacterial tests were done according to the JIS L 1902 standard [3]. For the antibacterial activity tests, $0.25 \mathrm{~g}$ of textile samples were inoculated with $200 \mu \mathrm{l}$ of $1-3$ $\times 10^{6} \mathrm{CFU}$ (colony forming unit)/ml of Escherichia coli bacteria. This concentration was determined via measurements with a WPACO 8000 Biowave personal cell density meter (Biochrom, UK) working at $600 \mathrm{~nm}$. The standard prescribes the use of an inoculum containing 1-3 $\times 10^{5} \mathrm{CFU} / \mathrm{ml}$, however this was not possible due to the optical density measurement limitations and therefore a slightly higher concentration was used. The testing standard describes the standard duration of an incubation as between 18-24 hours, this was modified to 1, 3, 6. 12 and 24 hours respectively.

PHMB and control samples were inoculated in the above described manner under sterile conditions. After the inoculation, the samples were placed on the inside of a petri dish lid and then covered with an inverted agar filled petri dish bottom. These plates were sealed with paraffin tape to prevent drying of the sample during the incubation. The samples were then placed in an incubator; operating at $37^{\circ} \mathrm{C}$ for the required duration $(1,3,6,12$, and 24 hours).

After the incubation period, the samples were taken out and rinsed in $20 \mathrm{ml}$ of physiological saline containing $2 \mathrm{~g} / 1$ Triton $\mathrm{X} 100.1 \mathrm{ml}$ from this rinsed solution was then pipetted out and serial diluted in $9 \mathrm{ml}$ of physiological saline. The serial dilution was done $0,10,100,1000$ and 10000 times. $0.1 \mathrm{ml}$ from these various serial diluted test tubes was then pipetted out and spread on an agar plate as shown in Figure 3.3. These plates were incubated overnight. 


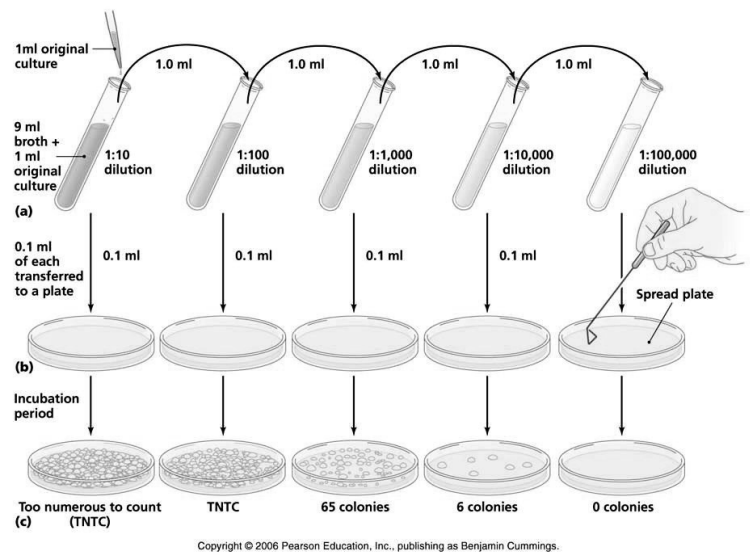

Figure 3.3 : The agar plate count method [13].

The next day, agar plates with colonies between 30-300 were selected for the counting. From the colonies counted, the serial dilution number and the rinsing liquid volume, the number of living bacteria on the textile was determined according to Equation 3.1 \& Equation 3.2.

Bacteria concentration $\left(\frac{C F U}{m l}\right)=\mathrm{N} \cdot \frac{\mathrm{D}}{\mathrm{v}_{\text {plating }}}$

Where $\mathrm{N}$ is number of colonies counted on the agar plate (between 30-300) and D stands for the dilution rate $\left(10^{0,1,2,3 \ldots}\right)$. V plating refers to the volume (in $\mathrm{ml}$ ) used for spreading of the liquid on the agar plate.

Number of $C F U$ per textile specimen $=$ Bacteria concentration. $\mathrm{V}_{\text {rinsing }}$

$\mathrm{V}_{\text {rinsing }}$ refers to the volume of saline used for rinsing the textile sample. In this work, this was $20 \mathrm{ml}$.

The amount of living bacteria on the untreated and treated samples at time $0\left(C F U_{0}^{*}\right.$ and $C F U_{0}$ ) was $1-3 \times 10^{6} \mathrm{CFU} / \mathrm{ml}$. This was determined by eluting the bacteria immediately from the samples after inoculation (without incubation step) and counting by the above described procedure.

The number of living bacteria is expressed in CFUs (or Colony Forming Units as explained in chapter 2). One CFU arises from one single living bacterial cell. This 
above described plate count method is the simplest way to count living bacteria on a textile sample. However this method has two disadvantages. One, this method cannot be used to count CFUs below 6000. CFUs below these numbers are considered to be zero. This is due to the fact that in this method only agar plates with colonies above 30 are selected for counting during the test. This requirement in the plate count method is for statistical reasons. Agar plates with colonies between 1-29 are considered zero. By substituting $\mathrm{N}$ as $30, \mathrm{D}$ as $10^{\circ}$ (therefore no serial dilution), $\mathrm{V}_{\text {plating }}$ as $0.1 \mathrm{ml}$ in Equation 3.1, and $\mathrm{V}$ rinsing as $20 \mathrm{ml}$ in Equation 3.2, it can be seen that 6000 CFUs is the lower measuring limit.

The second disadvantage with this method is that it is effective for counting only culturable bacteria i.e. those which can grow on agar plates. However there are bacteria that are viable but not culturable (VNBC). These are living bacteria with low metabolic activity and cannot be cultured on agar plates. The low metabolism of these bacteria is due to the result of stress created by the presence of the antibacterial agent. In time these bacteria resuscitate (as the concentration of the agent reduces in their vicinity) and then can be cultured on agar plates. These bacteria which cannot be enumerated by the plate count method, can be detected and counted by fluorescence methods. The plate count method therefore has the risk of underestimation of the number of living bacteria due to the above reason. Another reason that contributes to the underestimation is that sometimes a colony (CFU) arises out of clumping of two or more bacterial cells rather than a single cell. This is more so in the case of bacteria that grow in chains or clumps.

From the living bacteria eluted from the different samples, cultured and counted on the agar plate for the different incubation times, the antibacterial activity value, AA could be calculated, shown in Equation 3.3.

$A A=\left(\log C F U_{t}^{*}-\log C F U_{0}^{*}\right)-\left(\log C F U_{t}-\log C F U_{0}\right)$

Where CFU refers to the number of colony forming units of bacteria. The subscripts represent the incubation time, initially $t=0$ and final incubation time $t=1,3,6,12,18$, or 24 hours. The superscript * refers to the control samples; the treated sample is 
without the superscript *. This equation is the same as Equation 2.2. Here however, time $t$ covers a range of incubation times not the standard 24 hours.

\subsection{Eosin staining of PHMB treated cotton}

Eosin $\mathrm{Y}$ dye is a widely used staining dye for histology purposes. Eosin $\mathrm{Y}$ and polyhexamethylene biguanide are known to bind 1:1 stoichiometrically. Immersion of a PHMB treated textiles in a beaker containing Eosin $Y$ leads to the textile acquiring a fuschia colour. The concentration of Eosin $\mathrm{Y}$ dye molecules on the surface of the stained PHMB textiles is said to be proportional to the amount of the PHMB on the surface of the fabrics. This staining method could be a simple visual method to check if the PHMB application on the textile during the laundering process has been successful. This principle could also be used to derive a relation between the colour strength values of the treated textiles and the amount of PHMB on the textile. Since the actual amount of PHMB on the textile is not known, this correlation can be done between the applied PHMB bulk concentration and the colour strength values [14]. The colour strength values of stained/dyed textiles can be determined by a spectrophotometric reflectance meter.

An Eosin Y dye stock solution was prepared for the staining purpose. $50 \mathrm{ml}$ of 0.06 $\mathrm{w} / \mathrm{v} \%$ Eosin $\mathrm{Y}$ dye was dissolved in $350 \mathrm{ml}$ demi-water to which $50 \mathrm{~g}$ sodium citrate was added. The volume of the dye solution was then made up to $500 \mathrm{ml}$ with demiwater. $0.25 \mathrm{~g}$ of PHMB treated textile was put into a beaker containing $25 \mathrm{ml}$ of this dye solution and the dye solution was stirred for $10 \mathrm{~min}$ at room temperature. The sample was then taken out, rinsed in cold water and air dried. The same was done for the untreated textile or the control sample.

The colour strength of the samples were measured by the reflectance using a $\mathrm{X}$ rite colour spectrophotometer ( $X$ rite, Germany). The maximum absorbance of these stained samples was observed at $530 \mathrm{~nm}$. The measured reflectance data at this wavelength were used to calculate the absorption-scattering coefficient according to the equation of the Kubelka Munk shown below in Equation 3.4.

$$
\frac{\mathrm{K}}{\mathrm{S}}=\frac{(1-\mathrm{R})^{2}}{2 \mathrm{R}}
$$


Where $\mathrm{K}$ is absorption coefficient, $\mathrm{S}$ is scattering coefficient and $\mathrm{R}$ is the reflectance value.

\subsection{Results and discussion}

\subsubsection{Antibacterial activity tests}

The results of the antibacterial tests conducted on the different fabrics are shown in Figure 3.4. The amount of living bacteria recovered from fabrics is shown in the Log CFU scale on the y axis against the different PHMB bulk concentrations. The results show that at higher bulk concentrations treatments of the textile $(0.8 \mathrm{~g} / 1$ and $1 \mathrm{~g} / 1)$, no living bacteria is recovered from the sample. As expected it is seen that as the treatment bulk concentrations reduces, the living bacteria eluted increases.

In case of the control samples, the bacteria grow by a factor of 100 times over 6 hours and then the bacterial numbers appear to stabilize. This appears to be the characteristic growth curve of E coli on textile substrate in the current experimental conditions.

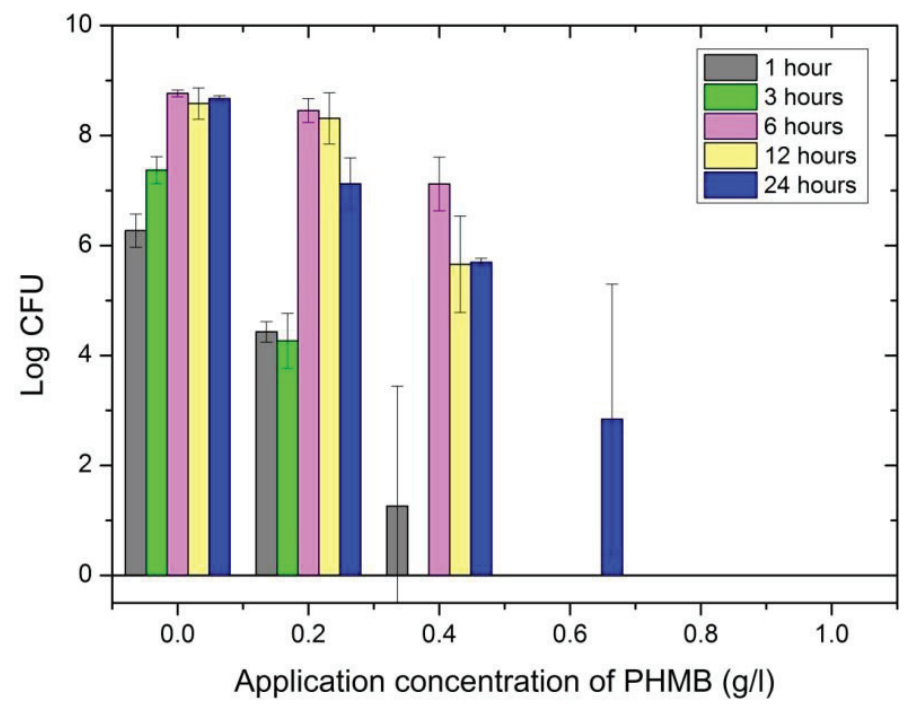

Figure 3.4: Amount of bacteria recovered from samples (in Log CFU) against different PHMB bulk concentrations (0-1 g/l). 


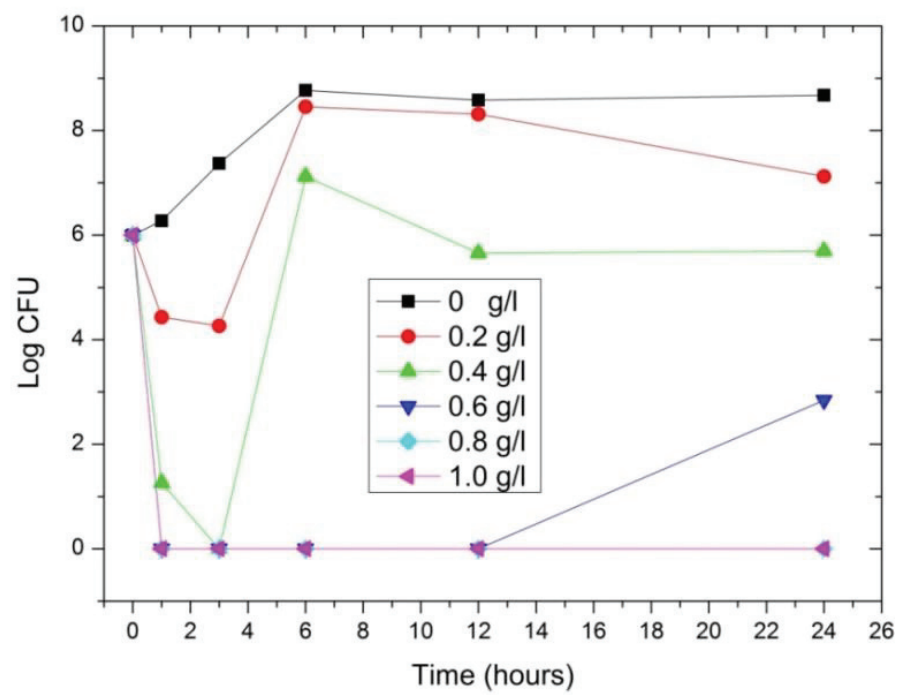

Figure 3.5: Amount of bacteria recovered from samples (in Log CFU) a function of incubation time for different PHMB concentrations.

Figure 3.5 shows the obtained CFU data as a function of the incubation time. After an initial reduction in the CFUs, an increase in the numbers is observed at lower bulk concentration treatments $(0.2 \mathrm{~g} / 1,0.4 \mathrm{~g} / \mathrm{l})$. This increase is observed in the growth phase of the bacteria (as seen from the control curve). This regrowth or increase in numbers is known to occur in situations when the agent concentration falls below the lethal limit or the Minimum Inhibitory Concentration (MIC, described in section 1.2.6 of chapter 1) [15]. This reduction in agent concentration with time occurs due to the agent uptake by the bacteria (as mentioned in section 1.2.3 of chapter 1 ).

When the agent concentration falls below the MIC, the attacked bacteria have a possibility to recover in time and resume their activity. It is known that bacteria treated with bacteriostatic levels of polyhexamethylene biguanide can recover despite having lost up to $40 \%$ of $\mathrm{K}^{+}$in their cells [14]. The damage to the cytoplasmic membrane is reversible at sub-lethal concentrations while above certain concentrations of the agent, recovery is not seen even when the agent has been removed from the vicinity [16]. At the high antibacterial agent concentrations, nucleotide leakage and cell the precipitation occur leading to permanent damage. 
The rate of the increase in bacterial numbers at sub-lethal concentrations can be said to be dependent on the doubling time of the microorganism or life phase of the bacteria (which is influenced by the nutrients in the environment) apart from the agent concentration in the vicinity.

No living bacteria were extracted at 3 hours of incubation for the PHMB bulk concentration treatment of $0.4 \mathrm{~g} / 1$ and at 12 hours incubation time of the PHMB bulk concentration treatment of $0.6 \mathrm{~g} / \mathrm{l}$. However, it is observed that with further increase in incubation time some living bacteria is eluted. The question then arises as to how any bacteria could multiply with increase in incubation time when all the bacteria were killed earlier. This can be explained by the earlier mentioned limitation regarding the plate count method where CFUs less than 6000 are considered to be zero. These zero CFU data points on the graph may represent colonies between 0 5999. These non-countable CFUs can multiply and then appear on the graph at later incubation times.

The antibacterial activity value calculated for all the treated samples for each incubation time is shown in Table 3.1. The calculated AA of the fabric depends on the several factors; substrate type (as explained in section 1.2.2 of chapter 1), amount of PHMB molecules on the surface of the fabric (as seen in Figure 3.3) which is steadily consumed during the antibacterial activity, the bacterial load, the growth phase of the bacteria (as seen from the growth curve of the control sample in Figure 3.3) and finally the strain of bacteria (as explained in section 2.1.3 of chapter 2). Real life conditions are quite different from laboratory conditions especially with regard to the environmental factors (such as moisture conditions and presence of nutrients). In real life the fabrics are also not wetted with such high bacterial loads (exceptions are with textiles used in surgical rooms and in butcheries). Therefore it can be concluded that fabrics that do pass laboratory tests will effectively work in real life.

From Table 3.1 it appears that the AA values of a treated fabric shows a rather dynamic trend depending on the incubation time. This is especially evident with fabrics treated with low PHMB bulk concentrations $(0.2 \mathrm{~g} / \mathrm{l} ; 0.4 \mathrm{~g} / \mathrm{l})$. These fabrics have the required AA of 2 at certain incubation times and fail to fulfill this criteria at other incubation times. 


\begin{tabular}{|c|c|c|c|c|c|}
\hline $\begin{array}{c}\text { PHMB bulk } \\
\text { concentration } \\
(\mathrm{g} / \mathrm{l})\end{array}$ & $\begin{array}{c}\text { AA in } \\
\mathbf{1} \text { hour }\end{array}$ & $\begin{array}{c}\text { AA in } \\
\text { 3 hours }\end{array}$ & $\begin{array}{c}\text { AA in } \\
6 \text { hours }\end{array}$ & $\begin{array}{c}\text { AA in } \\
12 \text { hours }\end{array}$ & $\begin{array}{c}\text { AA in } \\
24 \text { hours }\end{array}$ \\
\hline 0.2 & 1.84 & 3.11 & 0.31 & 0.27 & 1.55 \\
\hline 0.4 & 5.01 & 7.37 & 1.65 & 2.92 & 2.98 \\
\hline 0.6 & 6.27 & 7.37 & 8.77 & 8.58 & 5.83 \\
\hline 0.8 & 6.27 & 7.37 & 8.77 & 8.58 & 8.67 \\
\hline 1.0 & 6.27 & 7.37 & 8.77 & 8.58 & 8.67 \\
\hline
\end{tabular}

Table 3.1: Antibacterial activity of PHMB treated sample at different durations of incubation.

\subsubsection{Eosin staining tests}

The K/S measurements showed that there is a significant difference between untreated and PHMB treated samples as seen in Figure 3.4. However, the various PHMB treated samples do not show substantial differences in their respective $\mathrm{K} / \mathrm{S}$ values as seen from Table 3.2. It therefore appears that the K/S measurement method cannot be used to distinguish the various PHMB bulk treatments. However, this method can be used to check if any residue of the PHMB is left on the fabric after washing.

\begin{tabular}{|c|c|c|}
\hline $\begin{array}{c}\text { PHMB bulk } \\
\text { concentration }(\mathrm{g} / \mathrm{l})\end{array}$ & $\mathrm{K} / \mathrm{S}$ & $\begin{array}{c}\text { Standard } \\
\text { deviation }\end{array}$ \\
\hline 0 & 7.62 & 1.89 \\
\hline 0.2 & 14.30 & 0.23 \\
\hline 0.4 & 14.93 & 0.42 \\
\hline 0.6 & 15.14 & 1.74 \\
\hline 0.8 & 14.35 & 1.35 \\
\hline 1.0 & 14.34 & 2.08 \\
\hline
\end{tabular}

Table 3.2: K/S values for the Eosin $Y$ stained control and PHMB treated fabrics. The values are an average of 3 readings.

\subsection{Conclusion}

Cotton was treated with various bulk concentrations of polyhexamethylene biguanide. Antibacterial activity kinetics study was done with these treated samples following the Japanese standard against the E coli bacteria. Textiles treated with lower agent bulk concentrations showed a dynamic trend in the number of living bacteria eluted from the fabrics at the various incubation times. At higher bulk concentrations $(0.8-1 \mathrm{~g} / \mathrm{l})$, no living bacteria were extracted from the samples. The 
antibacterial activity values of the treated textiles showed that a treatment can fulfill the requirement for the minimum antibacterial activity at certain incubation times and fail to reach this limit at other incubation times.

\section{References}

1. Hugo, W. and S. Denyer, The concentration exponent of disinfectants and preservatives (biocides). Society for Applied Bacteriology. Technical Series, 1987. 22: p. 281-291.

2. Russell, A. and G. McDonnell, Concentration: a major factor in studying biocidal action. Journal of hospital Infection, 2000. 44(1): p. 1-3.

3. JIS (Japanese industrial standard) L 1902:Testing of antibacterial activity and efficacy on textile products, in Absorption method. 2002, Japanese Industrial Standard community: Tokyo.

4. Kawabata, A. and J.A. Taylor, The effect of reactive dyes upon the uptake and antibacterial efficacy of poly (hexamethylene biguanide) on cotton. Part 3: Reduction in the antibacterial efficacy of poly (hexamethylene biguanide) on cotton, dyed with bis (monochlorotriazinyl) reactive dyes. Carbohydrate polymers, 2007. 67(3): p. 375-389.

5. Gilbert, P. and L. Moore, Cationic antiseptics: diversity of action under a common epithet. Journal of applied microbiology, 2005. 99(4): p. 703-715.

6. Blackburn, R.S., et al., Sorption of poly (hexamethylenebiguanide) on cellulose: Mechanism of binding and molecular recognition. Langmuir, 2006. 22(13): p. 56365644.

7. Chen-Yu, J.H., D.M. Eberhardt, and D.H. Kincade, Antibacterial and Laundering Properties of AMS and PHMB as Finishing Agents on Fabric for Health Care Workers' Uniforms. Clothing and Textiles Research Journal, 2007. 25(3): p. 258.

8. Payne, J. and D. Kudner, A new durable antimicrobial finish for cotton textiles. American dyestuff reporter, 1996. 85(6): p. 26-30.

9. Wallace, M.L., Testing the efficacy of Polyhexamethylene Biguanide as Antimicrobial treatment for cotton fabric. AATCC Review, 2001. 1(11): p. 18-20.

10. Huang, W. and K.K. Leonas, Evaluating a one-bath process for imparting antimicrobial activity and repellency to nonwoven surgical gown fabrics. Textile Research Journal, 2000. 70(9): p. 774-782.

11. Chadwick, C., (Lonza group) personal communication. 2011.

12. http://www.lgcstandards-atcc.org. last accessed date: 27-10-14. 
13. http://spot.pcc.edu/ jvolpe/b/bi234/lec/4_growth/lecture_4L.htm. last accessed date: 5-12-2004.

14. Lonza Group Ltd (Arch chemicals), Reputex 20 technical brief.

15. Wu, V., A review of microbial injury and recovery methods in food. Food microbiology, 2008. 25(6): p. 735-744.

16. Broxton, P., P. Woodcock, and P. Gilbert, Injury and recovery of Escherichia ATCC 8739 from treatment with some polyhexamethylene biguanides. Microb 1984. 40(161-162): p. 187. 
Chapter 4 Single step functionalization of textile substrates 


\subsection{Introduction}

In chapter 1 , it was stated that the treatment of the fabrics can be done by the single step method or the two step/multi-step method which was an application based on the cyclodextrin host-guest system. The single step method of functionalization refers to the application where a textile is treated in a bath containing a solution of the antibacterial agent. Depending on the so called substantivity of the antibacterial agent with respect to the fabric, the bath will be exhausted in time. Therefore this simple method is also called the exhaust process.

As stated earlier, PHMB was the selected antibacterial agent for the single step method. This chapter describes the experiments done to determine the minimum PHMB bulk concentration required to produce an antibacterial textile in this single step method. As described in chapter 2, the value of the antibacterial activity (AA) of the treated textile is required to be at least 2 . The washing fastness tests of the treated antibacterial textiles is also presented in this chapter.

\subsection{Single step method experiments}

\subsubsection{Materials}

Cotton, blends and polyester substrates were chosen to be treated by the antibacterial agent. The reason for the choice of these substrates was that the work wear laundered in professional laundries are commonly made of these textiles. The cotton, blends and polyester used for these treatments were bought from WFK testgewebe $\mathrm{GmBH}$, Germany. They were all plain woven with a fabric density of $170 \mathrm{~g} / \mathrm{m}^{2}$. PHMB solution (Reputex 20) was sourced from Lonza group, UK. The details of this antibacterial agent in regard to its structure and the antibacterial mechanism has already been explained in chapter 3 .

\subsubsection{Experimental conditions for the single step method}

The experiments for the single step method were done by treating the three types of textiles with different concentrations of PHMB solutions. This was done to determine the minimum bulk concentration required to produce a textile with an AA of 2 . The treatment of the textiles was done in a beaker glass. The temperature was kept constant by placing the beaker on a thermostated plate. The bulk solution was 
continuous stirred with magnetic stirrers. The application conditions were fixed at temperature of $40^{\circ} \mathrm{C}$, and at $\mathrm{pH}$ of 7 based on the recommendations from Lonza, UK. These conditions are considered to be optimal for the exhaust process [1]. As explained in chapter 1 , the treatment was to be realized in the rinsing step of the laundering cycle in an industrial laundry. The treatment time for these experiments was based on the time available in the rinsing cycle. This was therefore chosen at 5 minutes. To check the influence of time on the antibacterial activity of the treated textiles, treatments were also done for 30 minutes. The LCR (Liquor to Cloth Ratio) was kept at 10:1. This was selected for practical reasons though the LCR in industrial laundries is at 5:1. After the treatment, the textiles were air dried.

The antibacterial tests of the treated samples were done according to the JIS L 1902 standard. These tests were done in a certified lab in Czech republic (Textile Testing Institute, Brno). The testing microorganisms were the gram positive Staphylococcus aureus CCM 4516 and gram negative Klebsiella pneumoniae CNCTC 6120. The antibacterial activity was then measured using Equation 2.2 described in chapter 2.

The washing fastness tests were done according to the ISO 105 C06 standard for colour fastness to domestic and commercial laundering [2]. For the washing experiments, the treated textiles were laundered in solution containing detergent in a linitester. An LCR of 35:1 was chosen as suggested in the standard. The temperature of washing was kept at $40^{\circ} \mathrm{C}$. A standard detergent without optical brighteners was used, the so called ECE reference detergent [3]. The concentration of this reference detergent in the washing liquor was $4 \mathrm{~g} / \mathrm{l}$. The surfactants in this detergent consisted mainly of anionic compounds. The composition of this detergent has been given below in Table 4.1 .

The treated samples were washed for 30 minutes after which the samples were rinsed for one minute in $100 \mathrm{ml}$ of $40^{\circ} \mathrm{C}$. After that the fabrics were then rinsed for one minute in a solution of $0.2 \mathrm{~g} / 1$ acetic acid at $30^{\circ} \mathrm{C}$. 


\begin{tabular}{|c|c|}
\hline Composition & Mass fraction \% \\
\hline Linear alkylbenzene sulfonate sodium salt & 8.0 \\
\hline Ethoxylated tallow alcohol & 2.9 \\
\hline Sodium soap & 3.5 \\
\hline Sodium tripolyphosphate & 43.7 \\
\hline Sodium silicate & 7.5 \\
\hline Magnesium silicate & 1.9 \\
\hline Carboxy methyl cellulose (CMC) & 1.2 \\
\hline Ethylene diamine tetra acetic acid(EDTA) & 0.2 \\
\hline Sodium sulfate & 21.2 \\
\hline Water & 9.9 \\
\hline
\end{tabular}

Table 4.1: The composition of the ECE reference detergent (without bleach, optical brighteners or enzymes).

This was done in order to neutralize the traces of the detergent alkalinity on the fabric. Finally the samples were finally rinsed again for one minute in $100 \mathrm{ml}$ of water at $30^{\circ} \mathrm{C}$ water. The fabrics were then dried for 30 minutes in an oven at $60^{\circ} \mathrm{C}$. After the laundering and the drying, the antibacterial activity of the samples were assessed by the lab in Czech Republic.

\subsection{Results and discussion}

Initially treatments with different PHMB bulk concentrations were done with only cotton samples. The treatment time here was kept at 5 minutes. The antibacterial activity, AA of these treated fabrics is shown in Table 4.2. From the table it is clear that only a bath concentration of $0.2 \mathrm{~g} / 1$ results in a fabric that meets the requirement of an AA of 2.

\begin{tabular}{|c|c|c|c|}
\hline $\begin{array}{c}\text { PHMB bulk concentration } \\
(\mathrm{g} / \mathbf{l})\end{array}$ & $\begin{array}{c}\text { Treatment time } \\
\text { (minutes) }\end{array}$ & $\begin{array}{c}\text { AA } \\
\text { Staphylococcus } \\
\text { aureus }\end{array}$ & $\begin{array}{c}\text { AA } \\
\text { Klebsiella } \\
\text { pneumoniae }\end{array}$ \\
\hline 0.05 & 5 & -0.70 & -0.11 \\
\hline 0.1 & 5 & -1.15 & -0.23 \\
\hline 0.2 & 5 & 3.71 & 3.91 \\
\hline
\end{tabular}

Table 4.2: The antibacterial activity of PHMB treated cotton samples. 
Thereafter experiments were performed with all the three substrates with two treatment times; 5 and 30 minutes with this bulk concentration. The results of these experiments have been summarized in Table 4.3 .

As can be seen from Table 4.3, a treatment time of 5 minutes is sufficient to achieve the minimum required AA for all the three substrates. It is also observed here that if the treatment time is increased to 30 minutes, an increase in antibacterial activity is seen for cotton and blends. However this is not the case for polyester.

\begin{tabular}{|l|c|c|c|c|}
\hline \multicolumn{1}{|c|}{ Bacteria } & Treatment time & $\begin{array}{c}\text { AA } \\
\text { Cotton }\end{array}$ & $\begin{array}{c}\text { AA } \\
\text { Blends }\end{array}$ & $\begin{array}{c}\text { AA } \\
\text { Polyester }\end{array}$ \\
\hline Staphylococcus aureus & 5 & 3.71 & 4.63 & 4.27 \\
\hline Staphylococcus aureus & 30 & 4.71 & 5.38 & 3.60 \\
\hline Klebsiella pneumoniae & 5 & 3.91 & 4.01 & 7.38 \\
\hline Klebsiella pneumoniae & 30 & 5.31 & 6.08 & 6.67 \\
\hline
\end{tabular}

Table 4.3: The antibacterial activity of $0.2 \mathrm{~g} / 1$ of PHMB treated cotton, blends and polyester samples.

The cationic biguanide groups in PHMB bind with the carboxylic acid groups of cotton via electrostatic interaction. The binding stoichiometric ratio between the carboxylic groups in cotton to PHMB is 1:1 [4]. The improvement in the antibacterial activity with time can be explained by the increase in the surface concentration of $\mathrm{PHMB}$ on the textile (this is further explained in chapter 5). The increase in the antibacterial activity in case of blends is due to the presence of cotton in the blends mixture. In case of polyester the antibacterial activity reduces slightly with an increase in the treatment time. The surface of polyester is inert without any reactive functional groups to allow covalent or electrostatic interaction (also referred to as chemisorption) with PHMB. Therefore only physical adsorption of PHMB onto to the surface of polyester occurs via the van der Waals forces. Physical adsorption is also known to occur much more quickly than chemisorption. This lack of any chemical interaction between PHMB and polyester surface leads to poor fixation of the molecule on the surface and thereby its removal may occur after a period of time. This could explain the reduction of the antibacterial activity of the PHMB treated polyester samples. 
Additionally it can be observed from the same table that AA of the treated textiles is higher against Klebsiella pneumoniae than that of Staphylococcus aureus for most of the cases. It therefore appears here that PHMB is more effective against the gram negative Klebsiella pneumoniae as compared to the gram positive Staphylococcus aureus. The gram negative bacteria differs from the gram positive in its structure of the cell wall and its permeability due to which it is less susceptible to various antibacterial agents [5]. In literature it is also reported that PHMB is more effective against gram positive bacteria than gram negative [6]. However, the results in this work appear contrary and the reasons are not known.

The results of the washing fastness tests are given below in Table 4.4. These tests were done on cotton, blends and polyester treated with $0.2 \mathrm{~g} / 1$ of PHMB for 5 minutes. As mentioned earlier, after the washing, antibacterial tests were done to determine the antibacterial activity of the samples.

From this table, it is clear that all the washed samples fail to pass the minimum required antibacterial activity value of 2 for Staphylococcus aureus as recommended by the Japanese standard.The AA of the cotton and blends samples against Klebsiella pneumoniae is above 2 while this is not the case for polyester.

\begin{tabular}{|c|c|c|}
\hline Substrates & $\begin{array}{c}\text { AA } \\
\text { Staphylococcus aureus }\end{array}$ & $\begin{array}{c}\text { AA } \\
\text { Klebsiella pneumoniae }\end{array}$ \\
\hline Cotton & 0.12 & 2.3 \\
\hline Blends & 0.29 & 2.2 \\
\hline Polyester & -0.16 & 1.1 \\
\hline
\end{tabular}

Table 4.4: The antibacterial activity of $0.2 \mathrm{~g} / 1$ of PHMB treated cotton, blends and polyester samples after washing tests.

These results show that after washing, a significant amount of PHMB is removed from the textile surface. The PHMB residue on the surface of the three substrates still shows some antibacterial activity against Klebsiella pneumoniae. This again confirms the earlier conclusion that PHMB shows better antibacterial activity against Klebsiella pneumoniae than Staphylococcus aureus. Though the washed cotton and blends samples show an antibacterial activity of above 2 with Klebsiella pneumoniae, the samples are considered to have failed the testing standard since it is required that the samples pass the minimum required AA for both the tested microorganisms. 
The reason for the poor washing fastness between PHMB and the cotton/blends substrates is due to the use of anionic based detergent. The cationic PHMB is deactivated by anionic detergents [1, 7]. In the literature good wash durability of PHMB treated samples has been reported $[8,9]$. The detergent type used in these washing tests reported in these articles is not specified. The reason for the good wash fastness of PHMB in these cases could be due to use of ionic detergents instead of anionic detergents.

From these experiments, it was concluded that a significant proportion of PHMB would be removed from the surface of a PHMB treated textile during the industrial laundering process if anionic detergents are used. This proportion of removal can be expected to be higher if a bleaching step is also included in the laundering process since PHMB is also deactivated by chlorine bleaches [1].

\subsection{Conclusion}

The treatment conditions and concentrations used for these experiments result in antibacterial fabrics that meet the minimum required antibacterial activity, AA of 2. A treatment time of 5 minutes in a bath with $0.2 \mathrm{~g} / 1$ of PHMB solutions at $40{ }^{\circ} \mathrm{C}$ and $\mathrm{pH}$ of 7 gives the required results. Cotton, blends and polyester samples fail to fulfil the AA criteria after one standard washing cycle. The washing cycle was done with an anionic detergent and the cationic PHMBs are deactivated by anionic detergents. Better PHMB washing fastness is expected in case of fabrics laundered with ionic detergents instead of the anionic type.

\section{References}

1. Lonza Group Ltd (Arch chemicals), Reputex 20 technical brief.

2. ISO 105 C06: Colour fastness to domestic and commercial laundering. 2010: Switzerland.

3. http://www.altraco.nl/content/altraco/producten/pdf/detergenten-testzeeppoeders.pdf. last accessed date: 5-12-2014.

4. Blackburn, R.S., et al., Sorption of poly (hexamethylenebiguanide) on cellulose: Mechanism of binding and molecular recognition. Langmuir, 2006. 22(13): p. 56365644. 
5. Denyer, S. and J.Y. Maillard, Cellular impermeability and uptake of biocides and antibiotics in Gram-negative bacteria. Journal of applied microbiology, 2002. 92(s1): p. 35S-45S.

6. Lee, W.R., et al., In Vitro Efficacy of a Polyhexamethylene Biguanide-Impregnated Gauze Dressing Against Bacteria Found in Veterinary Patients. Veterinary Surgery, 2004. 33(4): p. 404-411.

7. Simoncic, B. and B. Tomsic, Structures of Novel Antimicrobial Agents for TextilesA Review. Textile Research Journal, 2010. 80(16): p. 1721.

8. Payne, J. and D. Kudner, A new durable antimicrobial finish for cotton textiles. American dyestuff reporter, 1996. 85(6): p. 26-30.

9. Wallace, M.L., Testing the efficacy of Polyhexamethylene Biguanide as Antimicrobial treatment for cotton fabric. AATCC Review, 2001. 1(11): p. 18-20. 
Chapter 5 Textile adsorption and desorption

kinetics of PHMB 


\subsection{Introduction}

As explained in chapter 4, a textile substrate can be treated with an antibacterial agent in the single step method by soaking the textile in a solution of the antibacterial agent. The molecules of the antibacterial agent adsorb on to the textile surface from the bulk solution on the immersion of the textile. Simultaneously some of the adsorbed molecules desorb from the textile surface into the bulk solution. The kinetics of this adsorption and desorption process of the antibacterial molecule to and from the textile substrate can be modelled mathematically. This chapter describes a textile adsorption and desorption kinetics model of PHMB which has then been experimentally validated.

The adsorption of the antibacterial agent PHMB on to cotton has been described with a model in an earlier work [1]. In this work, cotton substrates were treated with PHMB solutions of different concentrations and the adsorption of PHMB on to the cotton surface was measured. The PHMB adsorption on to the cotton substrate followed Langmuir isotherm at low bulk concentrations. It was concluded that this implies site specific adsorption of PHMB molecules on the cotton surface. The attachment of PHMB to cotton is due to the electrostatic interactions which exist between the cationic biguanide groups of the PHMB (for the structure of PHMB refer to section 3.1.1 of chapter 3) and the carboxylate groups of cotton. These carboxylate groups on cotton are created during bleaching and or mercerization processes due to the oxidation of the glucose ring in the cellulose structure. The adsorption curves indicated that hydrogen bonding was also present between cotton and the PHMB molecules when the PHMB bulk concentrations were increased to higher concentrations. This was concluded from the derived adsorption isotherm constant which was characteristic of the Freundlich isotherm. Freundlich isotherm refers to the multilayer stacking of PHMB molecules on the cotton substrate during the exhaustion process (and hydrogen bonding is involved in this type of adsorption isotherm). Computer simulations showed that the hydrogen bonding was present between the biguanide units of PHMB and the oxygen, $\mathrm{C}-\mathrm{O}-\mathrm{C}$ and the hydroxyl groups of cotton. 
In contrast to the above mentioned work, this chapter describes the textile adsorption and desorption kinetics of PHMB with time. The PHMB fabric surface concentration is shown as a function of the adsorption and desorption rate constants in the mathematical model. Experimental work has been done to validate this theoretical model and then the adsorption rates and desorption rate constants have been determined. Finally the PHMB surface concentrations were recalculated using the determined rate constants.

\subsection{The kinetics of adsorption \& desorption}

As described earlier, the PHMB molecules are transferred from a bulk solution to the surface of the fabric during the adsorption process. The rate constant at which the molecules are adsorbed on to the textile can be referred to as $k_{\text {ads }}$. The PHMB molecules are transferred from the fabric surface back to the bulk solution at the same time and the rate constant of this desorption can be indicated by $k_{d e s}$. After a certain duration of time, an equilibrium is reached between the two processes. The general description of this is given below in Equation 5.1.

PHMB $B_{\text {bulk }} \stackrel{k_{\text {ads }}}{\stackrel{k_{d e s}}{\rightleftarrows}}$ PHMB $_{a d s}$

The above expression can be rewritten as shown below:

$C_{\text {bulk }} \stackrel{k_{\text {ads }}}{\stackrel{k_{\text {des }}}{\rightleftarrows}} \Gamma_{\text {fabric }}$

$C_{\text {bulk }}$ here denotes the concentration of PHMB molecules in the liquid bulk $\left(\mathrm{kg} / \mathrm{m}^{3}\right)$, $\Gamma_{\text {fabric }}$ is the concentration of PHMB molecules at the fabric surface $\left(\mathrm{kg} / \mathrm{m}^{2}\right), k_{\text {ads }}$ is the adsorption rate constant $(\mathrm{m} / \mathrm{s})$ and $k_{\text {des }}$ is the desorption rate constant $(1 / \mathrm{s})$. Based on Equation 5.2, the rate with which the change in the surface concentration with time can be given as:

$\frac{d \Gamma_{\text {fabric }}}{d t}=k_{a d s} C_{\text {bulk }}-k_{d e s} \Gamma_{\text {fabric }}$ 
The PHMB surface concentration can be related to the bulk concentration using the mass balance equation. The total amount of PHMB molecules in the system equals the amount in the bulk and the amount at the fabric surface:

$V_{\text {bulk }} C_{\text {bulk }, 0}=V_{\text {bulk }} C_{\text {bulk }}+A_{\text {fabric }} \Gamma_{\text {fabric }}$

Here $A_{\text {fabric }}$ refers to the total surface area of the fibres present in the fabric $\left(\mathrm{m}^{2}\right) . V_{b u l k}$ is the liquor bulk volume $\left(\mathrm{m}^{3}\right)$ or the volume of the exhaustion bath in which the adsorption takes place. $C_{b u l k, 0}$ is the initial concentration of PHMB molecules in the liquid bulk at time $t=0$. $C_{b u l k}$ can be expressed as:

$C_{\text {bulk }}=C_{\text {bulk }, 0}-\frac{A_{\text {fabric }}}{V_{\text {bulk }}} \Gamma_{\text {fabric }}$

The substitution of this bulk concentration expression in Equation 5.3 gives:

$\frac{d \Gamma_{\text {fabric }}}{d t}=k_{a d s}\left(C_{\text {bulk }, 0}-\frac{A_{\text {fabric }}}{V_{\text {bulk }}} \Gamma_{\text {fabric }}\right)-k_{\text {des }} \Gamma_{\text {fabric }}$

From which it follows that:

$\frac{d \Gamma_{\text {fabric }}}{d t}+\left(\frac{A_{\text {fabric }}}{V_{\text {bulk }}} k_{a d s}+k_{d e s}\right) \Gamma_{\text {fabric }}=k_{a d s} C_{\text {bulk }, 0}$

The general solution of this differential equation reads:

$\Gamma_{\text {fabric }}=\frac{k_{a d s} C_{\text {bulk }, 0}}{\frac{A_{\text {fabric }}}{V_{\text {bulk }}} k_{a d s}+k_{\text {des }}}+C e^{-\left(\frac{A_{\text {fabric }}}{V_{\text {bulk }}} k_{\text {ads }}+k_{\text {des }}\right) t}$

The integration constant $C$ can be found by applying the initial condition:

$t=0, \Gamma_{\text {fabric }}=\Gamma_{\text {fabric }, 0}$

$C=\Gamma_{\text {fabric }, 0}-\frac{-k_{\text {ads }} C_{\text {bulk }, 0}}{\frac{A_{\text {fabric }}}{V_{\text {bulk }}} k_{a d s}+k_{\text {des }}}$

with which the final solution of Equation 5.7 becomes:

$\Gamma_{\text {fabric }, t}=\frac{k_{\text {adso }} C_{\text {bulk }, 0}}{\frac{A_{\text {fabric }}}{V_{\text {bulk }}} k_{\text {ads }}+k_{\text {des }}}+\left(\Gamma_{\text {fabric }, 0}-\frac{k_{\text {ads }} C_{\text {bulk }, 0}}{\frac{A_{\text {fabric }}}{V_{\text {bulk }}} k_{\text {ads }}+k_{\text {des }}}\right) e^{-\left(\frac{A_{\text {fabric }}}{V_{\text {bulk }}} k_{\text {ads }}+k_{\text {des }}\right) t}$ 
This is the general model that describes the kinetics of adsorption and the desorption processes. The rate of adsorption and desorption can be calculated from the rate constants shown in equation. The values of the rate constants can be found from the experimentally determined adsorption and desorption curves.

\subsection{Textile adsorption and desorption experiments}

The adsorption and desorption curves of the PHMB to and from the textile substrates were obtained from the exhaustion experiments done of the various substrates with PHMB. The adsorption and desorption of PHMB to and from the textile could be followed by measuring the change in the concentration of PHMB in the bulk. In case of the adsorption experiments, the bulk refers to a liquor containing PHMB and in case of the desorption experiments, the bulk refers to a liquor containing only water. The measurement in the change of the PHMB concentration in the bulk was done via the (spectrophotometric) absorbance measurement of bulk liquor with a UV vis spectrophotometer.

\subsubsection{Materials}

Cotton, blends and polyester substrates were procured from WFK testgewebe $\mathrm{GmBH}$, Germany. These bleached substrates were plain woven. The fabric density was $170 \mathrm{~g} / \mathrm{m}^{2}$. The blends were a mix of cotton and polyester at 35:65 ratio. PHMB (Reputex 20) was procured from Lonza group, UK.

\subsubsection{Methods}

\subsubsection{The experimental conditions for the adsorption \& desorption experiments}

The adsorption and desorption studies were done on fabrics measuring $10 \mathrm{~cm}$ by 10 $\mathrm{cm}$. A bulk liquor containing PHMB was prepared using an LCR (Liquor to Cloth Ratio) of 100:1 for the adsorption experiments. The LCR used for the experiments in chapter 4 was 10:1. However for these studies such an LCR was not suitable for practical reasons (i.e. the low liquor volume in comparison to the fabric size was not convenient for the UV vis measurement). The experiments were done at a thermostated temperature of $40{ }^{\circ} \mathrm{C}$ [2]. The liquor in which the fabric samples were soaked was continuously stirred with magnetic stirrers. The results of the single step method experiments of chapter 4 indicated that $0.2 \mathrm{~g} / 1$ of PHMB bulk concentration 
resulted in fabrics with the required antibacterial activity value, AA of 2. However, this bulk concentration could not be used for these experiments due to the measuring limitation with the UV vis spectrophotometer (the absorbance was much above 1). These experiments were therefore conducted with a much lower PHMB bulk concentration, i.e. $0.05 \mathrm{~g} / 1$. Though the experimental conditions used in these experiments do not reflect the laboratory treatment conditions, they are however useful in understanding the textile adsorption and desorption kinetics of the PHMB molecule.

PHMB is a colourless solution with an UV vis absorbance peak at $236 \mathrm{~nm}$ [1]. During the adsorption experiments, $1 \mathrm{ml}$ of the bulk liquor was pipetted out and measured for its absorbance at $236 \mathrm{~nm}$ with the UV vis spectrophotometer (Cary 100, USA). This volume $(1 \mathrm{ml})$ was replaced into the bulk liquor after the absorbance measurement in order to keep the total bulk volume constant at all times. The duration of the experiments was kept at 100 minutes. By the end of 100 minutes, most of the PHMB in the bulk solution was found to have been adsorbed on to the textile. This was concluded from the low UV vis absorbance value of the bulk solution at the end of the experiment (the absorbance of PHMB in bulk at the end of the experiments was about $5-8 \%$ of the original absorbance). The absorbance measurements were made every minute during the first 10 minutes of the experiment, then every two minutes for the next 10 minutes and then every five minutes till the end of the experiment.

For the desorption experiments, a PHMB treated fabric with a known surface concentration of PHMB was soaked inside a bulk liquor containing water. The PHMB surface concentration of these fabrics was determined from the previously done adsorption experiments (just described above). These desorption experiments were done with an LCR of 100:1. The liquor in which the fabrics were placed was continuously stirred with magnetic stirrers. The temperature in these experiments was maintained at $40^{\circ} \mathrm{C}$ and the duration of the experiment was kept at 100 minutes. The absorbance of bulk liquor was measured at the earlier mentioned wavelength of $236 \mathrm{~nm}$. The bulk liquor measurement was made every minute the first 10 minutes, then every two minutes for the next 10 minutes and finally every five minutes till the end of the experiment. 
In the laboratory experiments, the PHMB textile treatments is done by placing a initially dry fabric into the exhaustion beaker. However, in industrial laundries the treatment is done when the fabric is in a wet state. The wet state of the fabric is due to the fact that the treatment is done in the rinsing step of the laundering cycle. It was therefore necessary to consider the influence of the initial dryness or wetness of fabric on the adsorption and desorption rate constants. Taking this difference into consideration the adsorption and desorption experiments were done in two different initial moisture conditions of the fabric; namely the wet to wet (W-W) and dry to wet (D-W) states of the fabric. The moisture condition of the fabrics here therefore refers to the initial state of dryness or wetness of the fabric before it is placed into the bulk liquor for the adsorption and desorption experiments. For the D-W tests, an initially dry fabric was placed inside the bulk liquor or water during the adsorption or desorption experiments respectively. For the $\mathrm{W}-\mathrm{W}$ tests, the fabric was made wet just prior to the experiment. This was done by placing the fabric in a beaker containing water for a few seconds and then the respective experiments were conducted.

\subsubsection{Fabric properties}

The fabric properties are an important parameter in the textile adsorption and desorption kinetics. The total surface area of all the fibres is therefore needed for the calculation of PHMB concentration at the fabric surface.

If $d_{\text {fibre }}$ is the fibre diameter and the total length of all the fibres in a piece of fabric is $L_{\text {fibre, }}$ the surface of the fabric $A_{\text {fabric }}$ reads:

$A_{\text {fabric }}=A_{\text {fibre }}=\pi d_{\text {fibre }} L_{\text {fibre }}$

$A_{\text {fabric }}$ here is the total surface of all the fibres in the fabric. This $A_{\text {fabric }}$ is also related to the weight of the fabric, the diameter of the fibres and the fibre density. The weight of the fabric, $W_{\text {fabric }}$, can be calculated by taking into consideration the volume and specific density of the fibres:

$W_{\text {fabric }}=\frac{1}{4} \pi d_{\text {fibre }}^{2} L_{\text {fibre }} \rho_{\text {fibre }}$

The fabric surface area is related to the weight of the fabric as shown below: 
$A_{\text {fabric }}=\pi d_{\text {fibre }} \frac{4 W_{\text {fabric }}}{\pi d_{\text {fibre }}^{2} \rho_{\text {fibre }}}$

or:

$A_{\text {fabric }}=\frac{4 W_{\text {fabric }}}{d_{\text {fibre }} \rho_{\text {fibre }}}$

Here $A_{\text {fabric }}$ therefore is the surface area of a fabric with a weight of $W_{\text {fabric }}$.

In this work, the average diameter of cotton and polyester fibers were measured with Scanning Electron Microscope (SEM). From the SEM images, it appeared that the cotton and polyester fibres did not have a uniform circular cross section throughout the length of the fibre or filament resectively. The diameter of the cotton fibre varied between 10-15 $\mu \mathrm{m}$. The filament diameter was between 10-13 $\mu \mathrm{m}$ in case of polyester. An average values of $13 \mu \mathrm{m}$ and $11.5 \mu \mathrm{m}$ were used for cotton and polyester fibres respectively in the model. As mentioned earlier, the blends were a mix of cotton and polyester with a ratio of $35 \%$ to $65 \%$. Based on this, the average fibre diameter for blends was taken at $12 \mu \mathrm{M}$.

Though the fibre/filament cross sections were not entirely uniform, we assumed them here to be perfectly cylindrical. From the respective fibre/filament diameter, density and weight of fabrics, the $A_{\text {fabric }}$ was calculated according to Equation 5.14 and these values are shown in Table 5.1.

\begin{tabular}{|c|c|c|c|}
\hline Parameters & Cotton & Blends & Polyester \\
\hline Fibre/filament diameter $(\mu \mathrm{m})$ & 13 & 12 & 11.5 \\
\hline Density $\left(\mathrm{kg} / \mathrm{m}^{3}\right)$ & 1520 & 1439.5 & 1380 \\
\hline$W_{\text {fabric }}(\mathrm{kg})$ & 0.00175 & 0.00166 & 0.00159 \\
\hline$A_{\text {fabric }}\left(\mathrm{m}^{2}\right)$ & 0.35 & 0.38 & 0.40 \\
\hline
\end{tabular}

Table 5.1: Table showing the fabric properties of the three substrates.

\subsubsection{Results and discussion}

\subsubsection{The experimental PHMB surface concentrations of cotton, blends \& polyester}

A PHMB calibration curve was made with the UV vis spectrophotometer. The calibration curve in Figure 5.1 shows the relationship between the UV vis absorbance against the various concentrations of PHMB in water at $236 \mathrm{~nm}$. The figure also 
shows the linear equation between these two variables. This equation was used to calculate the concentration of the PHMB in the bulk in the adsorption and desorption experiments.

From the calculated concentration of PHMB in the bulk and the $A_{\text {fabric }}$ values, the surface concentration of PHMB on the three substrates could be determined. The experimental surface concentration of PHMB as function of time on the three substrates is shown in Figure 5.2 and Figure 5.3 for the two different moisture conditions of the fabrics.

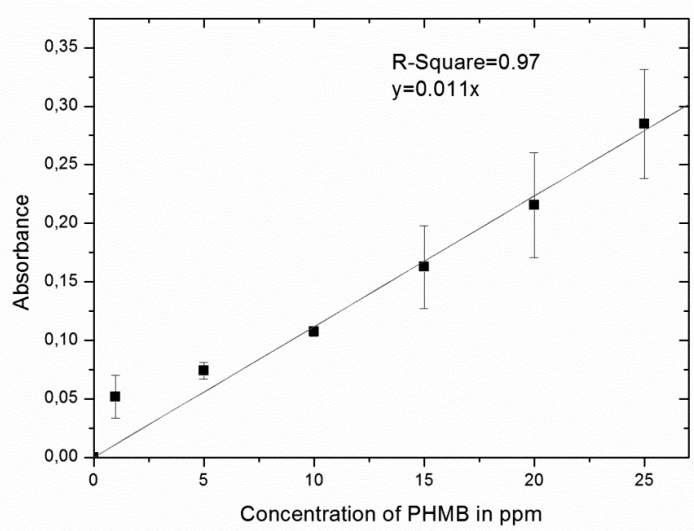

Figure 5.1:Calibration curve of PHMB with UV vis spectrophotometer. The unit for the concentration of PHMB here is expressed in ppm (parts per million) where $1 \mathrm{ppm}$ $=0.001 \mathrm{~g} / 1$.

The adsorption curves in Figure 5.2 and 5.3 clearly show that cotton has a higher PHMB surface concentration than blends and polyester. As mentioned earlier, PHMB molecules bind to the cotton surface via electrostatic interaction between the cationic biguanide groups and the anionic carboxylate groups of cotton. Therefore, the number of carboxylate groups on the fabric is a determining factor in the PHMB fixation amount apart from surface properties of the fabric. Blends theoretically have only $35 \%$ of the number of carboxylate acid groups contained in pure $100 \%$ cotton. This is due to the fact that blends are a mixture of $35 \%$ cotton to $65 \%$ polyester. Therefore the lower number of ionic sites in blends as compared to cotton would result in a lower PHMB surface concentration. 


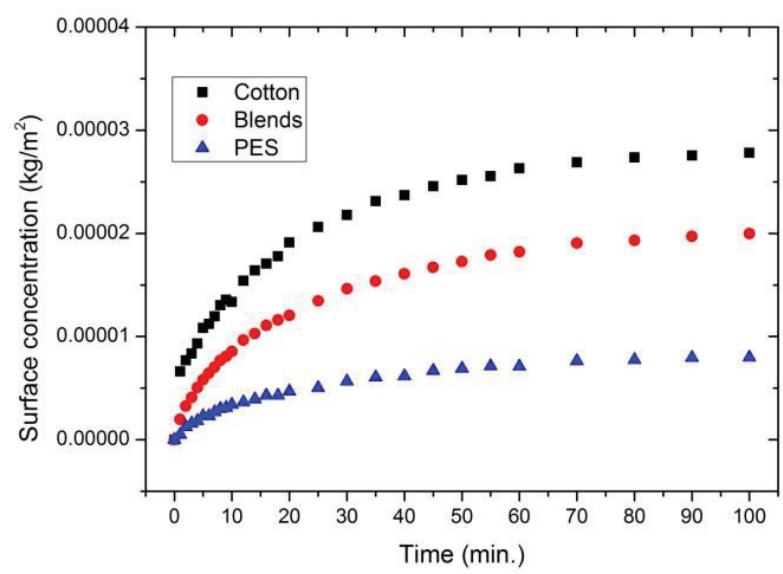

Figure 5.2: The surface concentration of PHMB on the three substrates in dry to wet (D-W) tests. Blends refer to a mix of cotton and polyester (35\% to $65 \%$ respectively). PES refers to polyester. The PHMB bulk concentration was $0.05 \mathrm{~g} / 1$.

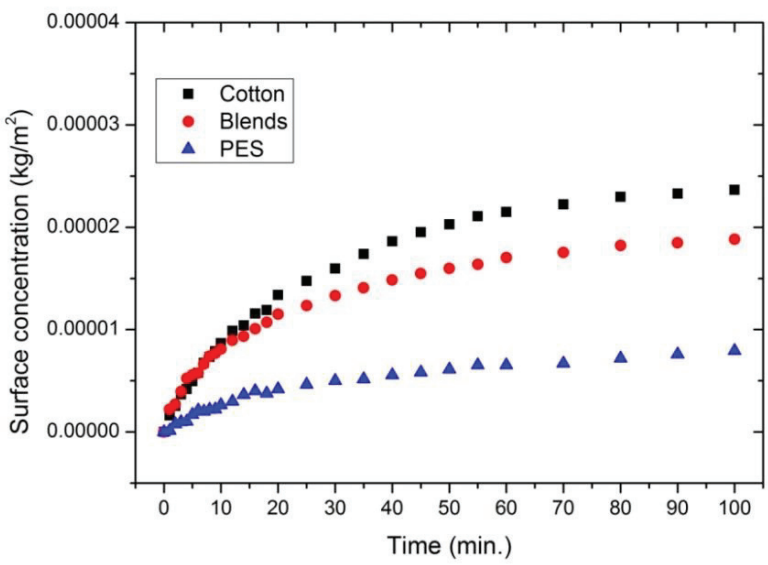

Figure 5.3: The surface concentration of PHMB on the three substrates in wet to wet $(\mathrm{W}-\mathrm{W})$ tests. Blends refer to a mix of cotton and polyester (35\% to $65 \%$ respectively). PES refers to polyester. The PHMB bulk concentration was $0.05 \mathrm{~g} / 1$.

As mentioned in chapter 4 , the surface of polyester has no reactive groups and therefore no covalent or ionic bonds are possible between the PHMB and the polyester substrate. The PHMB adsorption on to the polyester surface is therefore through physical adsorption. Polyester is also inherently hydrophobic due to the predominant hydrophobic aromatic and ethylene groups in its structure. As shown 
in chapter 3, the PHMB molecule contains polar biguanide groups and non-polar hexamethylene groups. Some hydrophobic interactions can be expected between the non-polar groups of PHMB and the polyester surface. Due to the lower affinity between the surface of polyester and the PHMB molecule, the surface concentration of the PHMB molecules on polyester is reduced.

From the above figures, it is also clear that the initial moisture condition of the fabric does not influence the adsorption of the PHMB molecules on to the fabrics in a significant way and this is evident more so in the case of polyester and blends.

The results of the desorption experiments are given below in Figure 5.4 and Figure 5.5. As mentioned earlier, the surface concentration of PHMB molecules on the treated textile was known prior to the desorption experiments. This was determined from the adsorption experiments. From the measured PHMB bulk concentrations and the $A_{\text {fabric }}$ values, the reduction in the PHMB surface concentration with time from the various fabrics was determined.

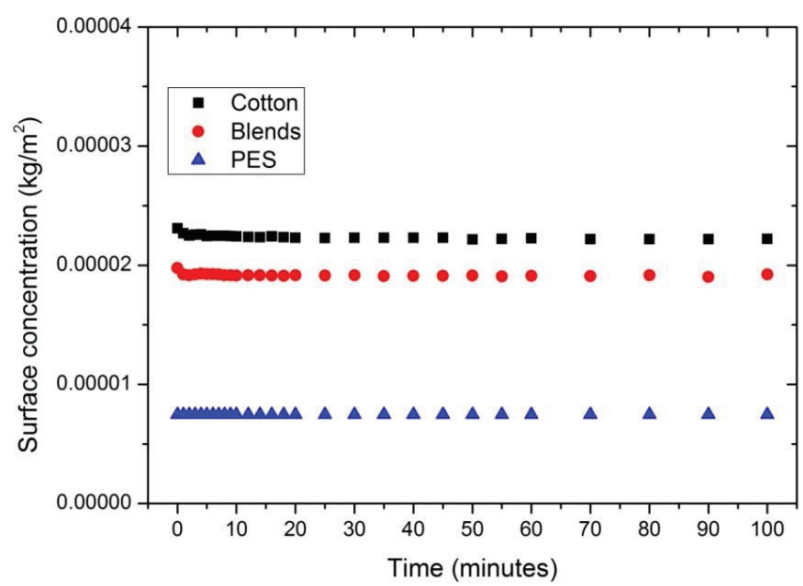

Figure 5.4: The desorption curves for the three substrates dry to wet (D-W tests). The three fabrics were treated with PHMB for 60 minutes prior to the desorption experiments. Blends refer to a mix of cotton and polyester mix of 35 to 65 ratio. PES refers to polyester. 


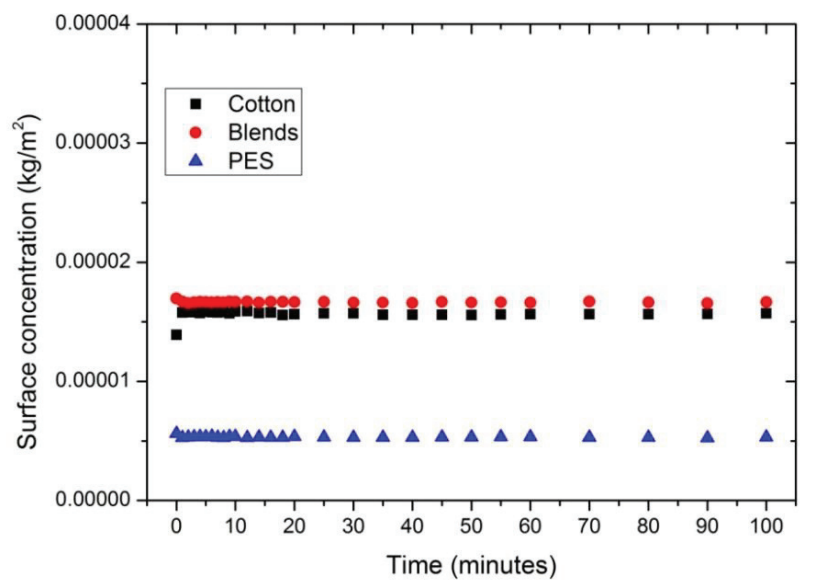

Figure 5.5: The desorption curves for the three substrates wet to wet (W-W tests). In this case the three fabrics were treated for 30 minutes prior to the desorption experiments. Blends refer to a mix of cotton and polyester mix of 35 to 65 ratio. PES refers to polyester.

The desorption experiments showed that the desorption of PHMB into the bulk from the three substrates (in both dry to wet or wet to wet scenarios) is nil. This meant that for further calculations related to the model validation, the influence of desorption can be excluded from the general equation (Equation 5.10).

\subsubsection{The adjusted model and determination of the adsorption rate constants}

As mentioned above, from the desorption experiments, it was concluded that the treated fabrics do not show desorption. If there is no desorption and when the initial fabric concentration is zero, i.e. if $k_{d e s}=0$ and $\Gamma_{\text {fabric }, 0}=0$, the general equation

(Equation 5.10) reduces to:

$\Gamma_{\text {fabric }, t}=\frac{k_{\text {ads }} C_{\text {bulk }, 0}}{\frac{A_{\text {fabric }}}{V_{\text {bulk }}} k_{\text {ads }}}-\frac{k_{\text {ads }} C_{\text {bulk }, 0}}{\frac{A_{\text {fabric }}}{V_{\text {bulk }}} k_{\text {ads }}} e^{-\left(\frac{A_{\text {fabric }}}{V_{\text {bulk }}} k_{\text {ads }}\right) t}$

This expression can also be shown as:

$\Gamma_{\text {fabric }}=\frac{V_{\text {bulk }} d_{\text {fibre }} \rho_{\text {fibre }}}{4 W_{\text {fabric }}} C_{\text {bulk, } 0}\left\{1-e^{-\left(\frac{4 W_{\text {fabric }} V_{\text {bulk }}}{d_{\text {fibre }} \rho_{\text {fibre }}} k_{\text {ads }}\right) t}\right\}$

Therefore the general equation has been modified to the above adsorption equation. 
By taking the system properties as:

$$
\mathrm{P}_{\text {system }}=\frac{V_{\text {bulk }} d_{\text {fibre }} \rho_{\text {fibre }}}{4 W_{\text {fabric }}}
$$

The equation is rearranged into:

$$
\ln \left(1-\frac{\Gamma_{\text {fabric }}}{P_{\text {system }} c_{\text {bulk }, 0}}\right)=-k_{\text {ads }} t / P_{\text {system }}
$$

A plot of $\ln \left(1-\frac{\Gamma_{\text {fabric }}}{P_{\text {system }} c_{\text {bulk }, 0}}\right)$ against time $t$ gives a straight line with the angle $-k_{\text {ads }} / P_{\text {system }}$ from which the $-k_{\text {ads }}$ can be calculated. The adsorption rate constants were derived in this manner for cotton and blends for the dry to wet and the wet to wet scenarios. Such a straight line could not be obtained for polyester due to which the adsorption rate constants for the polyester surface could not be determined.

The Table 5.2 shows the calculated adsorption rate constants for cotton and blends for both the dry to wet and the wet to wet cases.

\begin{tabular}{|c|c|c|}
\hline & $k_{\text {adsorption }}(\mathrm{m} / \mathrm{s})$ & \\
\hline Treatment condition & Cotton & Blends \\
\hline Dry to Wet & $5.62 \times 10^{-7}$ & $2.99 \times 10^{-7}$ \\
\hline Wet to Wet & $2.97 \times 10^{-7}$ & $2.87 \times 10^{-7}$ \\
\hline
\end{tabular}

Table 5.2: The calculated PHMB adsorption rates substrates for cotton and blends for the dry to wet and wet to wet scenarios.

The adsorption rate constants for blends in case of both the dry to wet and the wet to wet states are similar. It appears that the adsorption rate constants are not significantly influenced by the dryness or wetness state of the fabric during the treatment. The adsorption rate constant for cotton in the dry to wet state is twice the rate constant in case of wet to wet condition.

When a dry fabric is placed into the liquor, the adsorption is mainly driven by wicking while in the case of a wet fabric being treated in a liquor, the adsorption is primarily driven through diffusion. From the adsorption rate constants shown in 
Table 5.2, it appears that the diffusion of PHMB on to the textile fibres is already quite fast and it is irrespective of the initial dry or wet state of the fabric. This would imply that the use of mechanical energy would not significantly improve the diffusion any further and therefore mechanical agitation would not play an significant role in the adsorption process. This also suggests that this difference in rate constant for cotton between the wet and dry fabric will not have an significant influence on the PHMB uptake of the wet fabric during the rinsing stage of the laundering cycle since the rate constants are already rather quite high.

\subsubsection{The model PHMB surface concentrations on cotton \& blends}

From the experimentally determined adsorption rate constants and the general adsorption equation (Equation 5.16), the PHMB surface concentrations were recalculated. These surface concentrations are referred to as model surface concentrations in this chapter. Figure 5.6 and Figure 5.7 show the experimental PHMB surface concentrations and the model PHMB surface concentrations for cotton and blends for the dry to wet (D-W) and the wet to wet (W-W) conditions respectively.

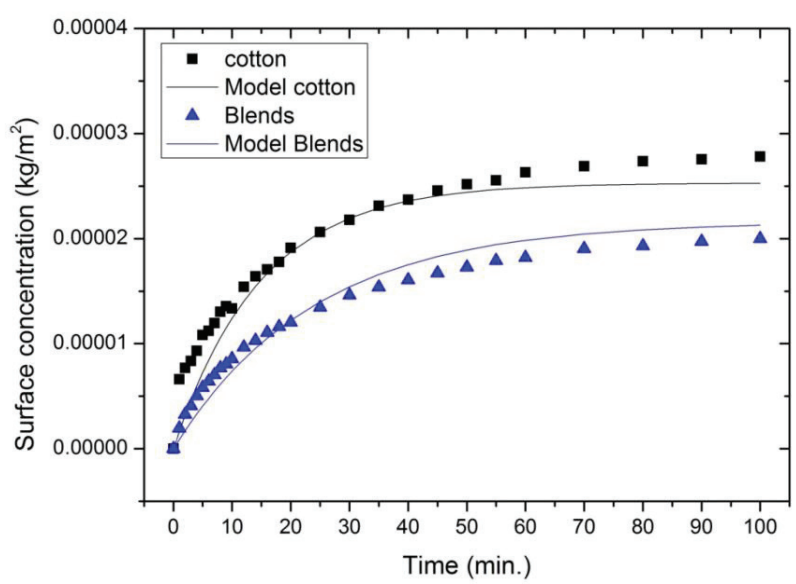

Figure 5.6: The experimental PHMB surface concentration versus the model PHMB surface concentration on cotton \& blends for the dry to wet (D-W) case. Blends refer to a mix of cotton and polyester (35\% to $65 \%)$. 


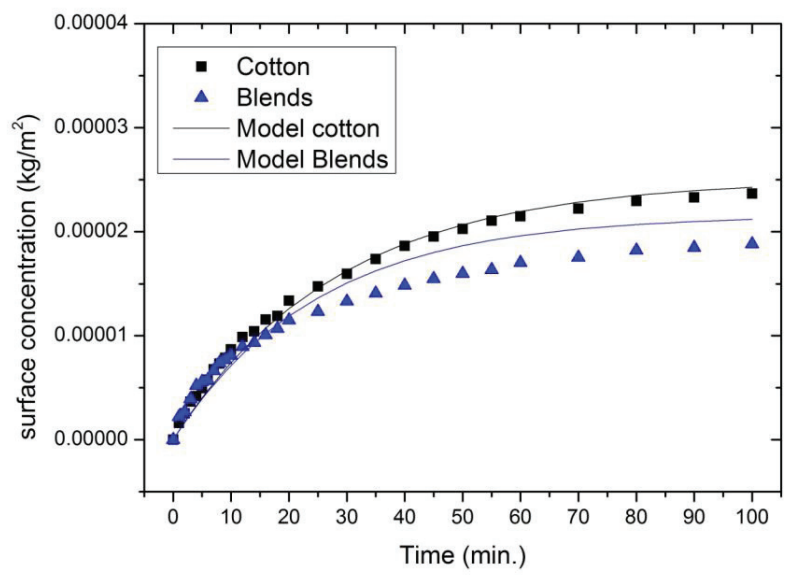

Figure 5.7: The experimental PHMB surface concentration versus the model PHMB surface concentration on cotton \& blends for the wet to wet $(\mathrm{W}-\mathrm{W})$ case. Blends refer to a mix of cotton and polyester (35\% to $65 \%$ ).

From the above shown figures, it appears that the model PHMB surface concentration curves and the experimental PHMB surface concentration curves are quite similar. From this it can be concluded that the theoretical model describes the adsorption kinetics of PHMB on to cotton and blends reasonably well. The only exception to the description of the model appears to be with the polyester substrate for which the adsorption rate constants could not be derived due to mathematical reasons.

\subsection{Conclusion}

The textile adsorption and desorption kinetics has been modelled mathematically by taking into consideration the adsorption-desorption processes and the fabric properties. The adsorption and desorption rate constants of the PHMB molecule on to the three textile substrates has been experimentally determined. No PHMB desorption was observed from the three substrate in the desorption experiments. The general model was then modified to exclude the influence of PHMB desorption from the substrates. The determined adsorption rate constants were then used to recalculate the model PHMB surface concentrations. The recalculated PHMB surface concentrations showed that the model describes the adsorption kinetics on cotton and blends quite well. The adsorption rate constants could not calculated for 
polyester substrate due to mathematical reasons and it was therefore concluded that this model was not applicable for the polyester substrate.

\section{References}

1. Blackburn, R.S., et al., Sorption of poly (hexamethylenebiguanide) on cellulose:

Mechanism of binding and molecular recognition. Langmuir, 2006. 22(13): p. 5636-5644.

2. Lonza Group Ltd (Arch chemicals), Reputex 20 technical brief. 
Chapter 6 Cyclodextrins 


\subsection{Introduction}

As described in chapter 1, the antibacterial functionalization of a textile can be done in a two-step process called the multi-step method as an alternative to the single step method. The first step in the multi-step method is the fixation of a host system to the surface of textile. Then the antibacterial molecule, also referred to as the guest molecule in this case is applied so that it is held inside the host molecule. As mentioned earlier in chapter 1, cyclodextrins were selected as the host molecules for the multi-step method and the main reason for the choice of cyclodextrins over other host molecules was their commercial availability. This chapter covers a review of cyclodextrins with the aim of describing their basic structure, uses and their fixation to various textile substrates.

Cyclodextrins are cyclic oligosaccharides composed of glucose units linked by a-1,4glycosidic bonds. The three most common type of cyclodextrins are; $a$-cyclodextrin (ACD), $\beta$-cyclodextrin (BCD) and $\gamma$-cyclodextrin (GCD) which are composed of 6,7 , and 8 a-1,4-glycosidic bonds or glucopyranose units respectively as shown in Figure 6.1. The general properties of these three types of cyclodextrins are shown in Table 6.1 .

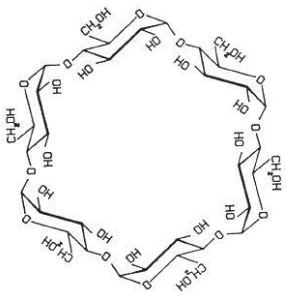

$$
\alpha C D
$$

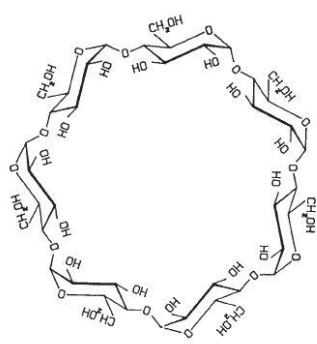

$\beta C D$

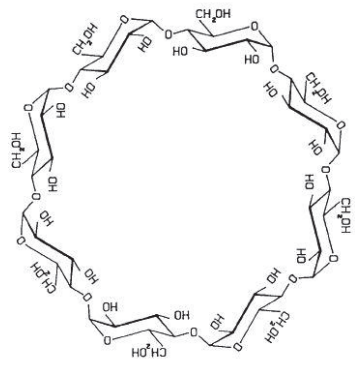

$\gamma C D$

Figure 6.1: The three main types of cyclodextrins. $\alpha C D, \beta C D$ and $\gamma C D$ refer to $\mathrm{ACD}, \mathrm{BCD}$ and GCD respectively.

Cyclodextrins are produced by the hydrolysis of starch with the use of cyclodextrin glucosyl transferase enzyme [3]. Carbohydrates ring structures can be arranged in boat or chair conformations depending on the plane in which the carbon atoms are positioned. In the chair conformation, the C-2, C-3, and C-5 atoms are in the same plane while C-1 lies above the plane and C-4 below the plane. 


\begin{tabular}{|c|c|c|c|}
\hline Properties & a-cyclodextrin & $\beta$-cyclodextrin & $\gamma$-cyclodextrin \\
\hline $\begin{array}{c}\text { Number of glucopyranose } \\
\text { units }\end{array}$ & 6 & 7 & 8 \\
\hline Molecular weight $(\mathrm{g} / \mathrm{mol})$ & 972 & 1135 & 1297 \\
\hline Solubility in water $(\mathrm{g} / \mathrm{l})$ & 145 & 18.5 & 232 \\
\hline Cavity diameter (Å) & $4.7-5.3$ & $6.0-6.5$ & $7.5-8.3$ \\
\hline Height of the torus $(\AA)$ & 7.9 & 7.9 & 7.9 \\
\hline
\end{tabular}

Table 6.1: The general properties of the three types of cyclodextrins $[1,2]$.

This type of conformation makes the molecule stable as compared to the boat conformation. Due to this chair arrangement of the glucopyranose units, cyclodextrins are conical cylinders with the secondary hydroxyl groups of the glucopyranose molecules (C-2 and C-3) on the wider side of the cylinder and the primary hydroxyls (C-6) on the narrow side of the cylinder. This conical cylinder structure is shown for BCD in Figure 6.2 The inner surface of the cavity is covered with glyosidic oxygen bridges and hydrogens (C-3 and C-5) of the glucose residues, giving the cavity a hydrophobic property while the outside of the cavity becomes hydrophilic due to the hydroxyl groups.

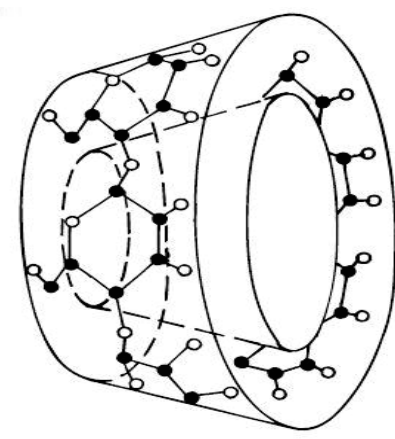

Figure 6.2: The truncated conical cylinder of BCD molecule.

In aqueous solutions, the hydrophobic cavity is occupied by water molecules and this is energetically unfavourable due to the apolar-polar interaction between the cavity and the water molecules. On the addition of lipophilic guest molecules, the water molecules in the cyclodextrin cavity are replaced with the hydrophobic molecules. In this way, the hydrophobic cavity of cyclodextrins can host several types of hydrophobic guest molecules. The cyclodextrin cavity hosting such a guest molecule is referred to as complex or specifically an inclusion complex and the 
process of formation of a complex is referred to as complexation. The complexation in the system is driven by the repulsive forces between the water molecules and the apolar guest. These repulsive forces exist between the included water molecules and the apolar guest in the cyclodextrin cavity as well as between the water molecules and the apolar guest molecules in the bulk [1].

Complexation of lipophilic guest molecules by the cyclodextrin cavities therefore leads to increase of the solubility of the guest molecule due to the solubilizing influence of hydrophilic exterior of the cyclodextrin molecule. Lipophilic guestcyclodextrin molecules are also known to form micellar like aggregates. In the pharmaceutical industry, most drugs need to be lipophilic to be able to permeate through biological membranes through passive diffusion. However, at the same time the drug formulations are required to have a certain degree of water solubility. The complexation of hydrophobic drugs with cyclodextrins is widely done to alter the solubility of poorly soluble drugs.

The cyclodextrin cavity is also known to protect the guest molecule from decomposition reactions induced by heat and light, oxidation or hydrolysis and from loss through evaporation [4].

As shown in Table 6.1, the cavity size of the three cyclodextrins is different from each other. This cavity size therefore determines the type of guest molecule that can fit inside it. ACDs have a relatively smaller cavity size due to which many guest molecules cannot be accommodated. GCDs on the other hand have a relatively large cavity. BCDs are found to accommodate a range of guest molecules from hormones, vitamins and drugs frequently used for tissue and cell culture applications [5]. Due to this BCD is widely used as a complexing agent. The drawback of BCD is its low water solubility as compared to ACD and GCD. This is due to the hydrogen bonding between the $\mathrm{OH}$ group at the $\mathrm{C}-2$ position of one glucopyranose units and the $\mathrm{OH}$ group at C-3 position of the adjacent glucopyranose unit. This hydrogen bonding is not present to the extent in ACD or GCD as it is in BCD [3]. The low water solubility of BCDs can be changed by modifying the structure of BCDs. Each glucopyranose unit of the BCD molecule has three hydroxyl groups and a single molecule of BCD contains 21 hydroxyl groups. BCDs can be modified by substituting the hydrogen 
atom or the hydroxyl groups of the molecule with a variety of groups such as amino, alkyl, hydroxyalkyl, carboxyalkyl, thio, glucosyl, ethers, esters groups, etc. This modification also called as derivatization can enhance the solubility of BCD and also improve the stabilization of a BCD-guest complex. This derivatization can be done by the substitution of all of the hydroxyl groups in the BCD molecule or with only the hydroxyl groups in certain positions of the glucopyranose unit. The hydroxyl group at C-6 position is most basic and nucleophilic while the one at C-2 is most acidic and the hydroxyl group at C-3 is most inaccessible. These factors along with reactivity of the agents influence the modification of the BCD molecule [6].

\subsection{BCD and guest complexes}

General conditions for obtaining a useful BCD-guest complex are that the molecular weight of the guest molecule should be between 100-400 g/ mol, the solubility of the guest in water should be below $10 \mathrm{~g} / \mathrm{l}$ and the guest should have less than five condensed rings. Additionally, the melting point of the guest molecule should preferably be below $250^{\circ} \mathrm{C}$ since it has been found that a high melting point of a guest molecule does not lead to a stable BCD-guest complex. Strongly hydrophilic, very small or very large molecules cannot be complexed inside the BCD cavity [7]. Depending on the size of the guest molecule, however, a partial complexation of the guest molecule is also possible. This is sometimes sufficient for the modification of guest molecule properties.

Most commonly a 1:1 complexation ratio is found between the guest and the BCD molecule while sometimes 2:2, 1:2 or 2:1 is also found [8]. No covalent bonds are formed or broken during the formation of such BCD guest complexes [9]. The complexation of BCDs with the guest molecules and the longevity of the complex is said to be determined by the electrostatic interactions, van der Waal forces, hydrophobic interactions and release of conformation strain (this is the energy stored in a molecule when under a strain) between the BCD and the guest molecules [8]. The molecular encapsulation is an equilibrium reaction characterized by an equilibrium or binding constant and the disassociation constant.

The reactivity, solubility, diffusion and volatility of the guest molecule is modified after the formation of a complex with the BCD cavities [3]. The spectral properties of 
the guest molecule (i.e. UV vis, NMR and fluorescence spectra) is also modified. Disassociation of such a complex is driven by the increase in number of water molecules in the surrounding environment [10]. Once a formed complex is placed in water, the complex dissolves and the guest molecules are displaced by water molecules. This continues till an equilibrium is reached between the free and complexed cyclodextrin.

\subsection{General industrial applications of BCDs}

As mentioned earlier, BCDs and BCD derivatives are widely used in the pharmaceutical industry. BCDs are used to complex a wide range of drugs in order enhance their solubility, dissolution rate, bioavailability and modify the drug release profile in the body. BCDs are also used to stabilize reactive ingredients. BCDs find applications in cosmetic industry, food industry, chemical, textile and paper industries. BCDs are used in products such as detergents, perfumes and room fresheners. Other applications are in personal care products such as toiletries, toothpastes, skin creams and dusting powders. In the food industry, BCDs are used to remove cholesterol from milk, butter and eggs. They are also used for flavour protection and flavour delivery [11]. BCDs also have applications in products for the agro industry such as pesticides, herbicides and insect repellents [9-11]. BCDs are also used in removal of organic pollutants and heavy metals from water and soil [12$14]$.

\subsection{Fixation of BCDs to textiles}

Fixation of BCDs to textiles, allows the possibility of treating textiles with a wide range of chemicals that may otherwise show little or no substantivity to the textile. This is due to the reason that the BCD cavity functions as a reservoir for the various guest molecules. BCD molecules cannot be directly attached to textile fibres since $\mathrm{BCDs}$ are not able to form covalent bonds with textile fibres. However the attachment can be done with the use of a crosslinker which can covalently link the BCD molecule to a textile fibre as shown in Figure 6.3. A widely used group of crosslinkers are the polycarboxylic acids. Polycarboxylic acids have been used to attach BCDs to cotton [15-17], wool [16, 17], polyester [17-19], polyamide [20] and polyacrylonitrile fibers [21]. Malic acid, citric acid, and 1, 2, 3, 4, 
butanetetracarboxylic acid (BTCA) are some examples of polycarboxylic acids. BTCA is one of the most commonly used polycarboxylic acids. BTCA is used in wrinkle resistant finish (also known as durable press finish) of cotton in the textile industry. It contains four carboxylic groups in its structure, one to three of which can react with the hydroxyl groups of cotton and BCD molecules in an esterification reaction [22].The esterification reaction between the acid and hydroxyl groups results in covalent ester groups thereby linking the cotton and the BCD molecules.

This esterification reaction is not possible between BTCA and polyester substrate due to the lack of reactive groups on the surface of polyester. However, BCDs can be physically fixed to polyester via the BTCA. The BCD molecules in this case are entangled with the physically fixed BTCA molecules of the polyester substrate [18]. This has also been observed in the BCD fixation experiments with polyester in this work (chapter 8). BCD derivates can also be fixed using these crosslinkers in the same manner. The two mostly commonly used BCD derivatives in the pharmaceutical industry are methyl $\beta$-cyclodextrin (Me BCD) and 2-hydroxypropyl $\beta$-cyclodextrin (HP BCD). Fixation of these two derivatives to textiles has been attempted with polycarboxylic acid crosslinkers [23, 24]. The main advantages with the use of BTCA as a crosslinker is the simple treatment procedure and as mentioned the already widespread use of BTCA in the textile industry which makes this process industrially viable.

In the past, resins such as epicholorohydrin have been reported to be employed as a crosslinker for the purpose of fixation $[25,26]$. This is a carcinogen and therefore is no longer considered for usage. Another widely used crosslinker for the wrinkle resistant finish in the textile industry is the dimethylol dihydroxyethyleneurea resin (DMDHEU).

This has also been used as a crosslinker to fix BCD to textiles [27]. However, this is a formaldehyde based crosslinker and therefore is considered to be toxic due to the carcinogenicity of formaldehyde. If colouration of the textile is not a limitation during the fixation of BCD to textiles, then heterobifunctional and homofunctional reactive dyes can be used as crosslinkers to fix $B C D$ to textiles in a conventional 
dyeing process [28]. Polyaminocarboxylic acid is the latest reported crosslinker for fixation of BCD to cotton [29].

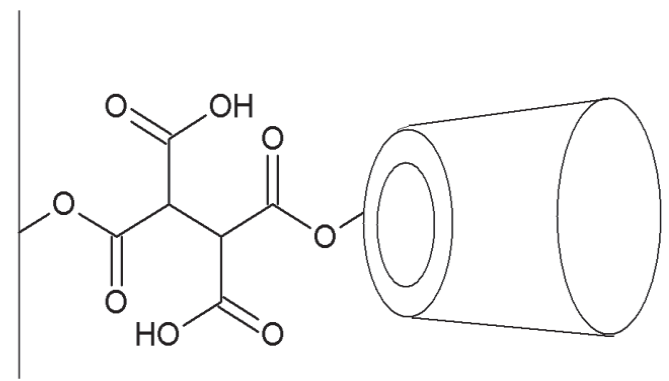

Figure 6.3: Grafting of BCD via BTCA to a textile [15].

The second way of attaching BCDs to various substrates is via graft polymerization. The initial step involves the creation of reactive radicals on the surface of any substrate. This process is referred to as initiation. Such surface initiation can be done by chemical methods or by radiation with plasma, UV light or electron beam. The surface is then treated with a solution of vinyl monomers such as glycidyl methacrylate which polymerize on to the textile surface [30]. These monomers are usually derivatives of acrylic acid and can polymerize on to surfaces quite easily due to their highly reactive double bonds. This grafted surface can then be linked to BCDs. As an alternative, a reactive BCD-acrylate polymer can also be polymerized in presence of an initiator and then attached to the textile surface [31].

A slight modification to the above method, is the fixation of BCDs to textiles by modifying the BCD structure such that it can directly react with the textile without the need for a crosslinker. These are referred to as reactive BCDs. One widely reported reactive $\mathrm{BCD}$ derivative is the monochlorotriazinyl- $\beta$-cyclodextrin (MCT $\mathrm{BCD})$. This derivative is synthesized by treating BCD with cyanuric chloride. Reactive dyes synthesized with monochlorotriazinyl groups can covalently fix for instance to cotton in alkaline conditions where the monochlorotriazinyl groups acts as a reactive anchor. This monochlorotriazinyl group is shown below in Figure 6.4. 
<smiles>[O-]Oc1nc(Cl)nc(Cl)n1</smiles>

Figure 6.4: Structure of monochlorotriazinyl functional group of MCT BCD molecule [32].

In case of MCT BCD fixation with cotton, the reactive chlorine atom of triazinyl groups of MCT BCD can react with hydroxyl groups of cotton and form a covalent bond through nucleophilic substitution as shown in Figure 6.5 [33].<smiles>Cc1nc(Cl)nc(O[NH3+])n1</smiles><smiles>CC=C[N-]C(N)(Cl)OCl</smiles>

Figure 6.5: Attachment of MCT BCD to cotton [34].<smiles>[18O]c1nc(O[AlH2])nc(O[123I])n1</smiles>

One other reactive $\mathrm{BCD}$ reported in literature is the $\beta$-cyclodextrin itaconate (BCDI) which is synthesized by treating BCD with itaconic acid [35]. Another example is acrylamidomethylated $\beta$-cyclodextrin (NMA BCD) [36]. Both these derivatives contain vinyl monomers which can polymerize on to the cotton surface with the use of chemical initiators such as ceric ammonium nitrate. An interesting BCD derivative is the BCD molecule with a functional tyrosyl group (Tyr BCD). In this case, tyrosinase enzyme is needed to create reactive quinone groups on the $\mathrm{BCD}$ derivative which can then be attached to cotton which has free aromatic amines on its surface. This can be achieved with a process called aminization where the cotton is dyed with a reactive dye and then reduced to leave free aromatic amine groups on its surface [28]. None of the above mentioned reactive derivatives are available commercially.

$\mathrm{BCD}$ derivatives with anionic, cationic or non-ionic properties can be made by the substitution of hydrogen or hydroxyl groups in the BCD molecule with cationic, 
anionic or non-ionic groups. It is reported that non-ionic BCD derivatives can be attached to hydrophobic textiles through their long hydrophobic alkyl or groups. Fixation of such derivatives to synthetic textiles such as polyamide, polyester and polyacrylonitrile through a process similar to disperse dyeing method has been attempted [34, 37, 38]. As mentioned earlier, the lack of commercial availability of also these non-ionic BCD derivatives make their application in the textile industry infeasible.

One unusual BCD fixation method to cotton in literature is via sol gel process with the use of tetraethoxysilane and 3-glycidyloxypropyl-trimethoxysilane [39].

However, it was observed that the stability of the gel solution was decreased by the addition of BCD. An alternative to exhaust finishing of BCDs on textiles is possible with the polymer extrusion process where BCDs can also be added to the synthetic pellet melt mixture $[40,41]$. However, this process is applicable only to melt extruded synthetic textiles.

\subsection{Quantification and characterization of BCD fixed on textile substrate}

Determination of BCDs can be done with HPLC and NMR spectroscopy. Among the various analytical methods, the photometric methods are more widely used. BCDs in solution do not show a UV absorbance peak and therefore cannot be directly detected in the exhaust or bulk solutions. BCD quantification on textile substrates can be done indirectly through absorbance measurements of dyes or indicators that can complex inside the BCD cavity. Some of these dyes are phenol red, phenolphthalein, methyl orange and iodine vapour [25, 42]. Phenolphthalein for instance is a widely used acid base indicator. It has a molar mass of $318.32 \mathrm{~g} / \mathrm{mol}$ and is very lipophilic. The complexation ratio between phenolphthalein and BCD is 1:1. Phenolphthalein is a weak acid which is colourless in acidic solutions (in benzenoid form) and pink in alkaline solutions (in quinoid form). The UV vis absorbance peak of an alkaline phenolphthalein solution is found to be at $550 \mathrm{~nm}$. On adding BCD to phenolphthalein solutions, its absorbance is found to reduce as the inclusion complex between phenolphthalein and BCD is formed (shown in Figure 6.6). 


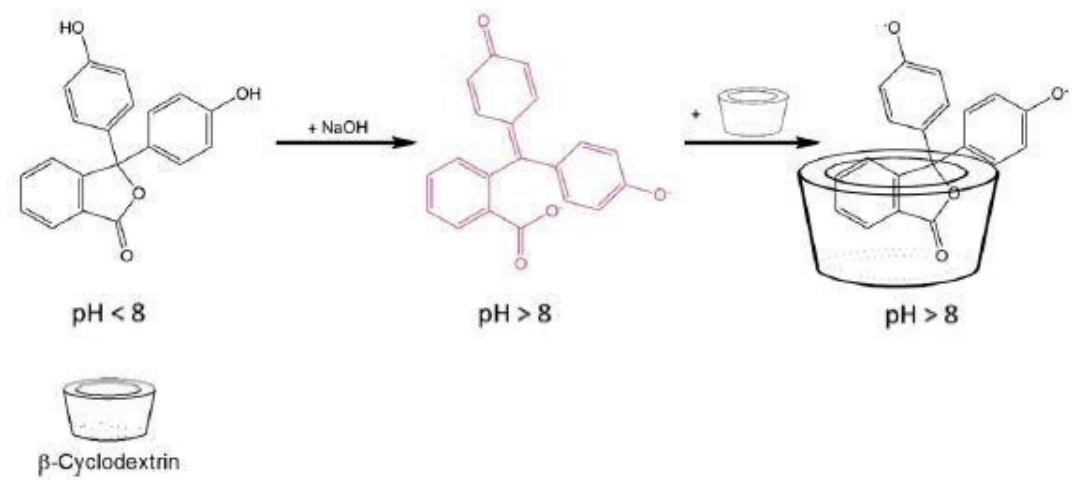

Figure 6.6: Complexation of phenolphthalein molecule inside the BCD cavity. The molecule shows pink colour in alkaline medium, the absorbance of which can be measured at $550 \mathrm{~nm}$. This absorbance reduces on complexation with BCD [43].

Another such indirect estimation method described in literature is via the complexation of fixed BCD with volatile aliphatic amines. In this case, BCD textile samples are exposed to volatile amines for a certain duration to allow the complexation of amines into the BCD cavities. The BCD treated fabric is then placed into water and the complexed amines are then extracted into water from these textile samples. The amount of these amines are then quantified through titration [44]. This method is time consuming as compared to the dye method. As mentioned earlier, a polycarboxylic acid crosslinkers can be used to attach BCD to the surface of a textile. In such a case, there is a possibility that the amines react with the free polycarboxylic acid on the fabric. This can lead to error in amine estimation [29], and consequently to the inaccurate estimation of the fixed BCD.

In case of fixation of a BCD derivative to a textile, the quantification of the derivative can be done by analyzing the elements or groups present in the derivative. An example is the quantification of MCT BCD through the triazine test method which is used to measure the amount of triazines in compounds [44] or the Kjeldahl method which is used to do measure the nitrogen content in compounds [45, 46]. However, these methods are considered to be not sensitive enough for precise quantification.

Solid state measurements of BCD treated fabrics has been reported in literature. Solid state UV vis spectroscopy characterization of BCD textiles can be done by monitoring the complexed organic volatile guest molecules such as toluene, cyclohexane or 
chlorobenzene [29], vanillin [47] or thymol [48] since BCD molecule itself does not show a observable peak against the textile background. The same applies to FTIRATR analysis of BCD textiles. Fluorescent dyes such as 1-anilinonaphthalene-8sulphonate, dansyl chloride, dansyl leucine can be used to determine BCDs using fluorescence spectroscopy [11]. Literature provides a few instances where this method was employed to characterize BCD treated textiles [34, 37, 38]. Diffuse reflectance infrared spectroscopy for quantification of $\mathrm{MCT} B C D$ on the textiles has been reported [49]. However, this method requires a calibration curve made from a reference analytical method. Research is currently ongoing in the quantitative assessment with Raman spectroscopy [50]. The advantage with these solid state measurements is that these tests are non-destructive and therefore the samples remain intact after the measurement.

\subsection{Applications of BCD treated textiles}

The earliest studies done with BCD treated textiles was in area of fragrance release. $\mathrm{BCD}$ attached fabrics and their control samples were treated with various aroma molecules and the release of their fragrance was qualitatively assessed over many days $[17,47,51-54]$. The studies show that BCD fixed fabrics showed increased shelf life of fragrances in comparison to the control samples. This was attributed to the modification of the volatility of the fragrance molecules when complexed inside the $\mathrm{BCD}$ cavities. This prolonged release of guest molecules, also referred to as slow, control or sustained release is characteristic of some BCD-guest complexes. This control release is also determined by the binding constant between the BCD and the guest molecule [55] apart from the modification in volatility as mentioned above.

Other interesting guest molecules for BCD textiles have been antimicrobial agents, sunscreen agents and insecticides [56-58]. Figure 6.7 shows the structures of some of these guest molecules. As mentioned earlier, these guest molecules fit the criteria required to complex with $\mathrm{BCD}$ in terms of their molar mass, geometry and hydrophobicity. 
<smiles>CCN(CC)C(=O)c1cccc(C)c1</smiles>

\section{$M, N$-diethyl-3-methylbenzamide}

Figure 6.7a: The mosquito repellent agent, N, N,-diethyl-3-methylbenzamide (also known as DEET). Molar mass $=191.27 \mathrm{~g} / \mathrm{mol}$.<smiles>Oc1cc(Cl)ccc1Oc1ccc(Cl)cc1Cl</smiles>

5-chloro-2-(2,4-dichlorophenoxy)phenol

Figure 6.7b: The antimicrobial agent Triclosan. Molar mass $=289.54 \mathrm{~g} / \mathrm{mol}$.<smiles>CCCCC(CC)COC(=O)/C=C/c1ccc(OC)cc1</smiles>

2-ethylhexyl (2E)-3-(4-methoxyphenyl)prop-2-enoate

Figure 6.7c: The sunscreen agent octyl methoxycinnamate. Molar mass $=290.4 \mathrm{~g} / \mathrm{mol}$.

One unusual application for BCD textiles is in the area of diagnostics. It has been suggested that these host attached textiles can be used to collect substances secreted by the skin [59] or to trap bad odours [60] which may be used for diagnostic analysis.

\subsection{Conclusion}

Cyclodextrins are cyclic organic compounds with a conical cylinder structure. The exterior of the conical cylinder is hydrophilic while the internal cavity is hydrophobic. This hydrophobic property of the cavity enables the molecule to host several types of lipophilic guest molecules. The attachment of cyclodextrins to textile surfaces allows treatment with chemicals that otherwise show little or no substantivity to the textile. There are several types of cyclodextrins among which $\mathrm{BCD}$ and $\mathrm{BCD}$ derivatives are considered to be most useful for various industrial 
applications. BCD derivatives are modified BCDs where the hydroxyl group or the hydrogen atoms of the glucopyranose units are substituted with other chemical groups. The simplest manner by which BCDs (or their derivatives) can be attached to a textile is through the use of polycarboxylic acid crosslinkers. BCD derivatives with reactive groups can also be synthesized. These reactive $\mathrm{BCDs}$ can be attached to a textile without a crosslinker. However, these are not commercially available and need to be synthesized in laboratories.

It was concluded based on this literature review that three types of BCDs would be attached to cotton to assess the most suitable type of BCD for the multi-step method. After the attachment, the quantification of the amount of BCD fixed on the substrate would be done with the phenolphthalein method. The selection of the most suitable type of BCD would then be done based on the highest amount of BCD fixation achieved on the textile surface.

The BCDs selected for the multi-step method were the unmodified BCD and two BCD derivatives namely HP BCD and MCT BCD. BTCA was selected as the crosslinker required for fixation of BCD and HP BCD to cotton. MCT BCD being a reactive derivative can be directly fixed to cotton without a crosslinker. The fixation of these BCDs, their quantification on the textile surface and the influence of the crosslinker BTCA on the fixation of BCD is described in detail in chapter 7.

In case of polyester a durable fixation with BCD via the use of a crosslinker or a reactive $B C D$ would not be possible due to the lack of reactive groups on the surface of polyester. A process of activation would be required to graft reactive groups after which polyester can be covalently fixed with BCD.

It was also concluded that PHMB is not a suitable guest molecule for the antimicrobial functionalization step of the multi-step method. This is due to its large structure (with a molar mass of $2750 \mathrm{~g} / \mathrm{mol}$ ) and its hydrophilicity. A molar mass of between 100-400 g/mol is recommended for the formation of BCD-guest complex. The high water solubility of $\mathrm{PHMB}$ is also a limitation considering that the $\mathrm{BCD}$ cavity is hydrophobic. Based on the complexation requirements, a new antimicrobial guest molecule was chosen for the multi-step method. This work is described in detail in chapter 8. 


\section{References}

1. Szejtli, J., Past, present and futute of cyclodextrin research. Pure and Applied Chemistry, 2004. 76(10): p. 1825-1845.

2. Loftsson, T., et al., Cyclodextrins in drug delivery. Expert opinion on drug delivery, 2005. 2(2): p. 335-351.

3. Szejtli, J., Introduction and general overview of cyclodextrin chemistry. Chemical reviews, 1998. 98(5): p. 1743-1754.

4. Buschmann, H.-J. and E. Schollmeyer, Applications of cyclodextrins in cosmetic products: a review. Journal of cosmetic science, 2002. 53(3): p. 185-192.

5. http://www.sigmaaldrich.com/technicaldocuments/articles/biofiles/cyclodextrins.html. last accessed date: 12-12-14.

6. Dodziuk, H., Cyclodextrins and their complexes: chemistry, analytical methods, applications. 2006: John Wiley \& Sons.

7. Szejtli, J., Utilization of cyclodextrins in industrial products and processes. Journal of Materials Chemistry, 1997. 7(4): p. 575-587.

8. Loftsson, T., et al., Cyclodextrins in drug delivery. Expert opinion drug delivery, 2005. 2(2): p. 335-351.

9. Singh, M., R. Sharma, and U.C. Banerjee, Biotechnological applications of cyclodextrins. Biotechnology advances, 2002. 20(5-6): p. 341-359.

10. Del Valle, E.M., Cyclodextrins and their uses: a review. Process Biochemistry, 2004. 39(9): p. 1033-1046.

11. Szejtli, J., Cyclodextrin technology. 1988: Springer.

12. Hirayama, F. and K. Uekama, Cyclodextrin-based controlled drug release system. Advanced drug delivery reviews, 1999. 36(1): p. 125-141. 
13. Hashimoto, H., Present status of industrial application of cyclodextrins in Japan. Journal of Inclusion Phenomena and Macrocyclic Chemistry, 2002. 44(1): p. 57-62.

14. Xia, Y. and J. Wan, Preparation and adsorption of novel cellulosic fibers modified by $\beta$-cyclodextrin. Polymers for Advanced Technologies, 2008. 19(4): p. 270-275.

15. Voncina, B. and A. Le Marechal, Grafting of cotton with $\beta$-cyclodextrin via poly (carboxylic acid). Journal of Applied Polymer Science, 2005. 96(4): p. 1323-1328.

16. Martel, B., et al., Polycarboxylic acids as crosslinking agents for grafting cyclodextrins onto cotton and wool fabrics: Study of the process parameters. Journal of Applied Polymer Science, 2002. 83(7): p. 1449-1456.

17. Martel, B., et al., Capture and controlled release of fragrances by CD finished textiles. Journal of Inclusion Phenomena and Macrocyclic Chemistry, 2002. 44(1): p. 439-442.

18. Martel, B., et al., Finishing of polyester fabrics with cyclodextrins and polycarboxylic acids as crosslinking agents. Journal of Inclusion Phenomena and Macrocyclic Chemistry, 2002. 44(1): p. 443-446.

19. Voncina, B., V. Vivod, and W. Chen, Surface modification of PET fibers with the use of $\beta$-cyclodextrin. Journal of Applied Polymer Science, 2009. 113(6): p. 3891-3895.

20. El Ghoul, Y., et al., Mechanical and physico-chemical characterization of cyclodextrin finished polyamide fibers. Journal of Inclusion Phenomena and Macrocyclic Chemistry, 2007. 57(1): p. 47-52.

21. Weltrowski, M., M. Morcellet, and B. Martel, Fiber with improved complexation qualities and cation exchange properties. 2006: United States.

22. El-Tahlawy, K., K. El-Nagar, and A. Elhendawy, Cyclodextrin-4 Hydroxy benzophenone inclusion complex for UV protective cotton fabric. Journal of the Textile Institute, 2007. 98(5): p. 453-462. 
23. Blanchemain, N., et al., Methyl- $\beta$-cyclodextrin modified vascular prosthesis: Influence of the modification level on the drug delivery properties in different media. Acta Biomaterialia, 2011. 7(1): p. 304-314.

24. Blanchemain, N., et al., Polyester vascular prostheses coated with a cyclodextrin polymer and activated with antibiotics: cytotoxicity and microbiological evaluation. Acta biomaterialia, 2008. 4(6): p. 1725-1733.

25. Szejtli, J., Cyclodextrins in the textile industry. Starch-Stärke, 2003. 55(5): p. 191196.

26. Szejtli, J., et al., Sorbents of cellulose basis capable of forming inclusion complexes and a process for the preparation thereof. 1982: USA.

27. Montazer, M. and M. Jolaei, $\beta$-Cyclodextrin stabilized on three-dimensional polyester fabric with different crosslinking agents. Journal of applied polymer science, 2010. 116(1): p. 210-217.

28. Agrawal, P.B., K. Ali, and M.M.C.G. Warmoeskerken, Inkjetable $\beta$-cyclodextrin based release system for cotton textiles, in Autex 2010 World Textile Conference. 2010: Vilnus, Lithuania.

29. Dehabadi, V., H.-J. Buschmann, and J. Gutmann, A novel approach for fixation of $\beta$-cyclodextrin on cotton fabrics. Journal of Inclusion Phenomena and Macrocyclic Chemistry, 2014. 79(3-4): p. 459-464.

30. Gawish, S., et al., Synthesis and characterization of novel biocidal cyclodextrin inclusion complexes grafted onto polyamide-6 fabric by a redox method. Journal of applied polymer science, 2006. 99(5): p. 2586-2593.

31. Hebeish, A., A. El Shafei, and S. Shaarawy, Synthesis and characterization of multifunctional cotton containing cyclodextrin and butylacrylate moieties. Polymer-Plastics Technology and Engineering, 2009. 48(8): p. 839-850. 
32. Kistamah, N., C.M. Carr, and S. Rosunee, Surface chemical analysis of tencel and cotton treated with a monochlorotriazinyl (MCT) $\beta$-cyclodextrin derivative. Journal of Materials Science, 2006. 41(8): p. 2195-2200.

33. Knittel, D. and E. Schollmeyer, Technologies for a new century. Surface modification of fibres. Journal of the Textile Institute, 2000. 91(3): p. 151-165.

34. Denter, U., et al., Modifizierung von Faseroberflächen durch die permanente Fixierung supramolekularer Komponenten, Teil 2: Cyclodextrine. Die Angewandte Makromolekulare Chemie, 1997. 248(1): p. 165-188.

35. Nazi, M., R.M.A. Malek, and R. Kotek, Modification of $\beta$-cyclodextrin with itaconic acid and application of the new derivative to cotton fabrics. Carbohydrate polymers, 2012.

36. Lee, M.H., K.J. Yoon, and S.W. Ko, Synthesis of a vinyl monomer containing $\beta$ cyclodextrin and grafting onto cotton fiber. Journal of applied polymer science, 2001. 80(3): p. 438-446.

37. Ruppert, S., et al., Fixierung von $\beta$-Cyclodextrinderivaten auf Polyesterfasermaterial. Starch-Stärke, 1997. 49(4): p. 160-164.

38. Denter, U. and E. Schollmeyer. Surface modification of synthetic and natural fibres by fixation of cyclodextrin derivatives. in Proceedings of the Eighth International Symposium on Cyclodextrins. 1996. Springer.

39. Wang, C.X. and S.L. Chen, Surface treatment of cotton using [beta]-cyclodextrins sol-gel method. Applied Surface Science, 2006. 252(18): p. 6348-6352.

40. Huang, L., E. Allen, and A. Tonelli, Inclusion compounds formed between cyclodextrins and nylon 6. Polymer, 1999. 40(11): p. 3211-3221.

41. Tonelli, A., The potential for improving medical textiles with cyclodextrin inclusion compounds. Journal of Textile and Apparel, Technology and Management, 2003. 3(2): p. 12. 
42. Denter, U. and E. Schollmeyer, Surface modification of synthetic and natural fibres by fixation of cyclodextrin derivatives. Journal of Inclusion Phenomena and Macrocyclic Chemistry, 1996. 25(1): p. 197-202.

43. AmeriáDehabadi, V. and J. StefanáGutmann, Spectrophotometric estimation of the accessible inclusion sites of $\beta$-cyclodextrin fixed on cotton fabrics using phenolic dyestuffs. Analytical Methods, 2014. 6(10): p. 3382-3387.

44. Grechin, A.G., H.-J. Buschmann, and E. Schollmeyer, Quantification of cyclodextrins fixed onto cellulose fibers. Textile Research Journal, 2007. 77(3): p. 161-164.

45. Hebeish, A. and Z.H. El-Hilw, Chemical finishing of cotton using reactive cyclodextrin. Coloration Technology, 2001. 117(2): p. 104-110.

46. Abdel-Halim, E.S., et al., Chitosan and monochlorotriazinyl-[beta]-cyclodextrin finishes improve antistatic properties of cotton/polyester blend and polyester fabrics. Carbohydrate Polymers, 2010. 82.

47. Nakpathom, M., B. Somboon, and S. Changpradit, Fragrant Finishing of Cotton Fabric Using $\beta$-Cyclodextrin. Advanced Materials Research, 2008. 55: p. 909-912.

48. Rukmani, A. and M. Sundrarajan, Inclusion of antibacterial agent thymol on $\beta$ cyclodextrin-grafted organic cotton. Journal of Industrial Textiles, 2012. 42(2): p. 132-144.

49. Heise, H., et al., Quantitative diffuse reflectance infrared spectroscopy of cotton fabrics treated with a cyclodextrin derivative finishing auxiliary. Journal of molecular structure, 2005. 744: p. 877-880.

50. Heise, H., et al., Infrared spectroscopy and Raman spectroscopy of cyclodextrin derivatives and their ferrocene inclusion complexes. Vibrational Spectroscopy, 2010. 53(1): p. 19-23.

51. Wang, C. and S. Chen, Surface modification of cotton fabrics with $\beta$-cyclodextrin to impart host-guest effect for depositing fragrance. Vol. 4. 2004: American Association of Textile Chemists and Colorists. 
52. Wang, C. and S. Chen, Aromachology and its application in the textile field. Fibres Textiles East. Eur, 2005. 13(6): p. 41-44.

53. Wang, C.X. and L.S. Chen, Fragrance-release Property of $\beta$-Cyclodextrin Inclusion Compounds and their Application in Aromatherapy. Journal of Industrial Textiles, 2005. 34(3).

54. Sricharussin, W., et al., Modification of cotton fabrics with $\beta$-cyclodextrin derivative for aroma finishing. Journal of the Textile Institute, 2009. 100(8): p. 682-687.

55. Roquette, Technical sheet Kleptose Betacyclodextrins and hydroxypropyl betacyclodextrins.

56. Wang, J.-h. and Z. Cai, Incorporation of the antibacterial agent, miconazole nitrate into a cellulosic fabric grafted with $\beta$-cyclodextrin. Carbohydrate polymers, 2008. 72(4): p. 695-700.

57. Scalia, S., et al., Incorporation of the sunscreen agent, octyl methoxycinnamate in a cellulosic fabric grafted with $\beta$-cyclodextrin. International journal of pharmaceutics, 2006. 308(1): p. 155-159.

58. Hebeish, A., et al., Preparation of durable insect repellent cotton fabric: Limonene as insecticide. Carbohydrate polymers, 2008. 74(2): p. 268-273.

59. Knittel, D., K. Beermann, and E. Schollmeyer, Surface of textiles and the human skin. Exogenous dermatology, 2003. 2(1): p. 17-22.

60. Buschmann, H.-J., D. Knittel, and E. Schollmeyer, New textile applications of cyclodextrins. Journal of inclusion phenomena and macrocyclic chemistry, 2001. 40(3): p. 169-172. 
Chapter $7 \quad$ Attachment of unmodified $\beta$-cyclodextrin and $\beta$-cyclodextrin derivatives on cotton 


\subsection{Introduction}

\subsubsection{Selection of $\beta$-cyclodextrins}

As stated in chapter 1 and chapter 6, $\beta$-cyclodextrins were selected as the host molecules for the multi-step method. The $\beta$-cyclodextrins fixed on to the surface of textiles function as loading baskets for the various guest molecules. The inclusion of the guest molecule into the cavity of the host molecule was to be realized in the rinsing step of the laundering cycle. As mentioned in chapter $6, \beta$-cyclodextrins are available in their unmodified form or as derivatives. $\beta$-cyclodextrins and some of their derivatives can be attached to textiles via a polycarboxylic crosslinker. However, reactive $\beta$-cyclodextrins can be fixed directly to a textile without the use of a crosslinker.

This aim of the work described in this chapter was to select the most suited type of $\beta$ cyclodextrin for fixation to textiles. The criteria for this assessment was to be based on the amount of the $\beta$-cyclodextrin fixation obtained after the exhaust treatment of the textile with the various $\beta$-cyclodextrins. As discussed in conclusion of chapter 6, the three types $\beta$-cyclodextrins that were chosen for these fixation experiments were the unmodified $\beta$-cyclodextrin (BCD), a derivative form of $\beta$-cyclodextrin and a reactive $\beta$-cyclodextrin. The derivative form of $\beta$-cyclodextrin selected was $2-$ hydroxypropyl- $\beta$-cyclodextrin (HP BCD) and the reactive $\beta$-cyclodextrin selected was monochlorotriazinyl- $\beta$-cyclodextrin (MCT BCD). The fixation of these three $\beta$ cyclodextrins was done on cotton.

In this chapter, the acronym BCD is used while referring to a specific type of $\beta$ cyclodextrin and the term $\beta$-cyclodextrins is used while referring to the all of the three types used in this work.

The unmodified $\beta$-cyclodextrin (BCD) is also called as native or parent $\beta$-cyclodextrin in the literature. It's structure is described in section 6.1 of chapter 6. One of the most widely used derivatives of BCDs is $\mathrm{HP} \mathrm{BCD}$. This derivative is obtained by the substitution of the hydroxyl groups of the anhydroglucopyranose units of the BCD molecule with 2- hydroxypropyl groups. Due to the substitution with these hydrophilic 2-hydroxypropyl groups in $\mathrm{HP} \mathrm{BCD}$, the molecular weight and the 
solubility of this derivative increases to $1480 \mathrm{~g} / \mathrm{mol}$ and $500 \mathrm{~g} / 1$ respectively from $1135 \mathrm{~g} / \mathrm{mol}$ and $18.5 \mathrm{~g} / \mathrm{l}$ of the unmodified BCD [1].

As already explained in chapter 6, MCT BCD is a reactive derivative in which the hydroxyl groups of the anhydroglucopyranose units of the BCD are substituted with monochlorotriazinyl groups. The substitution with these aromatic triazine rings results in an increase in molecular weight of the derivative to $1560 \mathrm{~g} / \mathrm{mol}$ from 1135 $\mathrm{g} / \mathrm{mol}$ of the unmodified form [2]. The aqueous solubility of MCT BCD is more than $300 \mathrm{~g} / 1[3]$.

\subsubsection{Selection of crosslinker, catalyst and curing conditions}

BCD and HP BCD were fixed to cotton via a polycarboxylic acid crosslinker 1, 2, 3, 4, butanetetracarboxylic acid (BTCA). Polycarboxylic acid crosslinkers are a group of widely used durable press or crease resistant finish crosslinkers in the textile industry. The crease resistant finishes with polycarboxylic acids is achieved by crosslinking of the cellulose chains via a esterification reaction. BTCA is the most effective among the various polycarboxylic acid crosslinkers for crease or wrinkle resistant finishes. Some other examples of polycarboxylic crosslinkers are malic acid, succinic acid, tartaric acid, itaconic acid, citric acid, etc.

BTCA has four carboxylic acid groups present in its structure compared to the two carboxylic acid groups in malic acid or succinic acid and the three acid groups in citric acid. One to three of these carboxylic acid groups in BTCA are involved in this esterification reaction with cotton. The esterification of BTCA with cotton occurs in two steps, first by the formation of an five membered cyclic anhydride through the dehydration of the adjacent carboxyl groups and then followed by the formation of ester links between the anhydride intermediate and cellulose [4]. In case of malic acid or succinic acid which are bifunctional polycarboxylic acids, only one carboxyl group is said to be involved in esterification with cotton [5]. Citric acid is cheaper than BTCA and more eco-friendly, however, it is known to cause yellowing of fabrics [6]. The capacity of BTCA to form higher number of ester linkages leads to not only good crease resistant finish but also to high wash durability of the finish. 
One other reason for choice of BTCA was that at certain application concentrations, as reported in some literature, BTCA is known to give flame resistant properties to blends [7] and to cotton [8], i.e. class 1 flame retardant effect according to the ASTM D1230 test method. Therefore, it was envisaged that using BTCA might also give flame retardant properties to the host attached fabrics.

BCD can be attached to cotton using BTCA which reacts with the hydroxyl groups of $\mathrm{BCD}$ and cotton via the carboxylic acid groups of BTCA through the earlier mentioned esterification reaction. Figure 7.1 shows this mechanism schematically. This esterification reaction has been validated by the FTIR scans of cotton attached to BCD via BTCA by Medronho et. al [9]. As mentioned in chapter 6, a single BCD molecule contains 21 hydroxyl groups. However the reactivity of all of these hydroxyl groups towards esterification is not the same. The hydroxyl groups at C-6 position of the glucopyranose unit is said to be the most nucleophilic in neutral and in acidic conditions and therefore more inclined to participate in this esterification reaction than the hydroxyl groups at C-2 or C-3 positions.

A catalyst, the right curing temperature and curing time are also required for effective crosslinking via a esterification reaction. Sodium hypophosphite (SHPI) is a widely used catalyst with BTCA. It speeds up the process of esterification of BTCA with cotton by weakening the hydrogen bonds between the carboxylic groups and reduces the cyclic anhydride formation temperature as compared to the use of BTCA alone [10]. SHPI is also considered as an optimum catalyst for BTCA especially in terms of the durability of the crease resistant finish [11, 12].

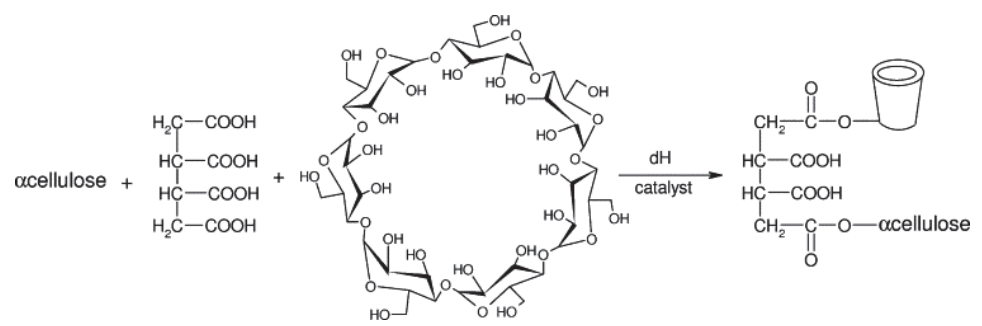

Figure 7.1: Attachment of BCD to cellulose with BTCA [9]. The cellulose in the figure can be replaced with cotton which is essentially $90 \%$ cellulose.

The temperature of curing in this work was kept at $160^{\circ} \mathrm{C}$ since it is known that the anhydride formation in the BTCA molecule with SHPI occurs at this temperature 
[13]. The use of higher temperatures could mean more crosslinking of BTCA, however this also leads to loss in tensile strength of the treated textile [14]. In the literature, the use of BTCA to SHPI of 3:1 [10], 2:1 [15], 1:1 [16] mass ratios and 1:0.3 molar ratio are reported [17]. According to a US patent, SHPI as a catalyst can be effective starting from $0.3 \%(3 \mathrm{~g} / 1)$ to $11 \%$ [18]. Since it is known that only a small amount of catalyst is sufficient to be effective in chemical reactions, the BTCA to SHPI was taken at 1:0.3 molar ratio for this work [17].

\subsection{Attachment of $\beta$-cyclodextrins to cotton}

\subsubsection{Materials}

The plain woven bleached cotton of fabric density $170 \mathrm{~g} / \mathrm{m}^{2}$ was obtained from WFK Testgewebe, Germany. The BCD and HP BCD were obtained from Roquette, France. The average number of hydroxypropyl groups substituted per BCD molecule in the HP BCD procured for our work is 5.6. BTCA, sodium hydroxide, sodium carbonate, Tris(hydroxymethyl) aminomethane (Tris), phenolphthalein and SHPI were obtained from Sigma Aldrich. The MCT BCD produced by Wacker chemie was procured from CTD Inc, USA. The MCT BCD contains 2-3 reactive groups per BCD molecule. MCT $\mathrm{BCD}$ at this moment is no longer in production.

\subsubsection{Fixation with the different types of $\beta$-cyclodextrin}

The fixation experiments were done with the three different types of $\beta$-cyclodextrins by varying the application concentrations from $40 \mathrm{~g} / 1$ to $100 \mathrm{~g} / 1$. Cotton fabrics were exhaust treated at room temperature with a Liquor to Cloth Ratio (LCR) of 20:1. The treatment was done on fabrics measuring $10 \mathrm{~cm}$ by $10 \mathrm{~cm}$. The other variables in these experiments were the concentrations of the crosslinker BTCA (10 g/l, $30 \mathrm{~g} / 1$ and $60 \mathrm{~g} / \mathrm{l})$, curing temperature $\left(160^{\circ} \mathrm{C}\right.$ and $\left.180^{\circ} \mathrm{C}\right)$ and BTCA to the catalyst SHPI ratio (1:0.3 molar ratio and 1:1 molar ratio). For all the fixation experiments, the respective control samples were prepared (i.e. without the addition of any $\beta$ cyclodextrin).

\subsubsection{Fixation of BCD on to cotton}

The treatment liquor or bulk volume was calculated based on the LCR and the weight of the sample. BTCA (10, 30 or $60 \mathrm{~g} / 1)$ and SHPI were added into the bulk 
and the solution was magnetically stirred till the chemicals were completely dissolved. The $\mathrm{pH}$ of this bulk was around 3. Then the required amount of BCD was added (0-100 g/l) into the bulk and dissolved. Though the solubility of BCD is only $18.6 \mathrm{~g} / \mathrm{l}$, higher concentrations of BCD could be easily dissolved in the liquor due to the low $\mathrm{pH}$ or acidity of the bulk liquor. Fabric samples were then placed inside the liquor and magnetically stirred for 5 minutes at room temperature. After the exhaust treatment, the fabric samples were taken out and pre-dried at $110^{\circ} \mathrm{C}$ for 10 minutes. This was followed by curing in an oven at $160^{\circ} \mathrm{C}$ for 5 minutes. The samples were then first rinsed in demi water for 5 minutes at $60^{\circ} \mathrm{C}$, then again under running tap water and finally air dried. These experimental conditions were considered as the standard exhaust conditions.

The influence of curing conditions and the influence of the catalyst on the amount of $\mathrm{BCD}$ fixed on cotton was further assessed. For these fixation experiments the curing temperature was increased to $180^{\circ} \mathrm{C}$. The BTCA to SHPI ratio was also then increased to 1:1 molar ratio from the standard 1:0.3 molar ratio. The BCD fixation amount obtained has then been compared to the amount achieved using the standard exhaust conditions $\left(160^{\circ} \mathrm{C} ; 1: 0.3\right.$ molar ratio of BTCA to SHPI).

\subsubsection{Fixation of HP BCD on to cotton}

For the treatment with HP BCD (0-100 g/l), the same procedure was followed as described above with the exception that the amount of BTCA used was only $30 \mathrm{~g} / 1$. This concentration of the crosslinker was selected based on the results of BCD fixation experiments. No experiments with change in curing conditions were done.

\subsubsection{Fixation of MCT BCD on to cotton}

The treatment conditions for attachment of MCT BCD were taken from literature published by Wacker chemie [3, 19]. For the treatment with MCT BCD $(0-100 \mathrm{~g} / \mathrm{l})$, the procedure was the same as with BCD fixation with the exception that no BTCA/SHPI was added and the treatment was done in alkaline medium ( $\mathrm{pH} 11 ; 125$ $\mathrm{mM}$ sodium carbonate solution). The treatment time, pre-drying, curing at $160{ }^{\circ} \mathrm{C}$ and sample rinsing after the treatment were the same as with BCD and HP BCD fixation. 


\subsection{Quantification of $\beta$-cyclodextrins fixed on cotton}

The quantification of $\beta$-cyclodextrins fixed on cotton was done via the gravimetric measurements and the phenolphthalein method.

\subsubsection{Gravimetric method}

The amount of $\beta$-cyclodextrins fixed on cotton was measured gravimetrically according to Equation 7.1. The cotton samples were conditioned at $20^{\circ} \mathrm{C}$ and $65 \%$ relative humidity for 24 hours before the measurements.

$W \%=\frac{W f-W b}{W b} .100 \%$

Where $W b$ is the weight of the sample before the fixation and $W f$ is weight of the sample after the fixation.

\subsubsection{Phenolphthalein method}

The quantification of $\beta$-cyclodextrin can be done indirectly using an acid base indicator such as phenolphthalein. As described in chapter 6 an alkaline phenolphthalein solution has a pink colour and shows an UV vis absorbance peak at $550 \mathrm{~nm}$. A reduction in this absorbance peak is observed when phenolphthalein molecules complex with $\beta$-cyclodextrin molecules. An absorbance-concentration relationship between the phenolphthalein and the $\beta$-cyclodextrin can be obtained with a UV vis spectrophotometer. This principle is used to determine the amount of $\beta$-cyclodextrin fixed on a textile surface.

A $4 \mathrm{mM}$ solution of phenolphthalein was made in ethanol. $1 \mathrm{ml}$ of this solution was mixed with $4 \mathrm{ml}$ of ethanol and $95 \mathrm{ml}$ of $125 \mathrm{mM}$ of sodium carbonate solution. The concentration of phenolphthalein and that of sodium carbonate used for this method were taken from the literature $[20,21]$. The $\mathrm{pH}$ of this $0.04 \mathrm{mM}$ phenolphthalein solution was $11.4 \mathrm{ml}$ of this phenolphthalein working solution was added to $1 \mathrm{ml}$ of buffer solution (phenolphthalein-buffer solution). The tris buffer solution prepared was of $0.05 \mathrm{M}$ concentration and of $\mathrm{pH}$ 7. $\beta$-cyclodextrin treated fabrics were then cut to a sample sizes of $2.5 \mathrm{~cm}$ by $2.5 \mathrm{~cm}$. These samples were then put in the $5 \mathrm{ml}$ of phenolphthalein-buffer solution. The final concentration of phenolphthalein in this 5 $\mathrm{ml}$ phenolphthalein-buffer solution was $0.032 \mathrm{mM}$. These test tubes containing the 
treated samples were covered with an aluminum foil and were kept for 2 hours in a dark cabinet. The covering with the aluminum foil was done as a precaution against any photo oxidation that phenolphthalein can undergo. The 2 hours of storage gave enough time for the complexation to come to an equilibrium state. Then the UV vis absorbance of the solution was measured at $550 \mathrm{~nm}$ using Cary $100 \mathrm{UV}$ spectrophotometer (Agilent Technologies, US).

For this method a calibration curve is also needed in order to obtain the curve fit equation describing the absorbance-concentration relationship between phenolphthalein and the $\beta$-cyclodextrins. A series of $\beta$-cyclodextrin-phenolphthalein test tubes were set up. Each test tube contained $1 \mathrm{ml}$ of varying concentrations of $\beta$ cyclodextrins ( $\mathrm{C}, \mathrm{g} / \mathrm{l})$ made in the buffer solution (Tris buffer solution; $0.05 \mathrm{M}$; $\mathrm{pH} 7$ ), and $4 \mathrm{ml}$ of the $0.04 \mathrm{mM}$ phenolphthalein solution. The UV vis absorbance of these solutions were measured and a curve fit equation was obtained. The calibration curves for the three different $\beta$-cyclodextrins is given below in the Figure 7.2.

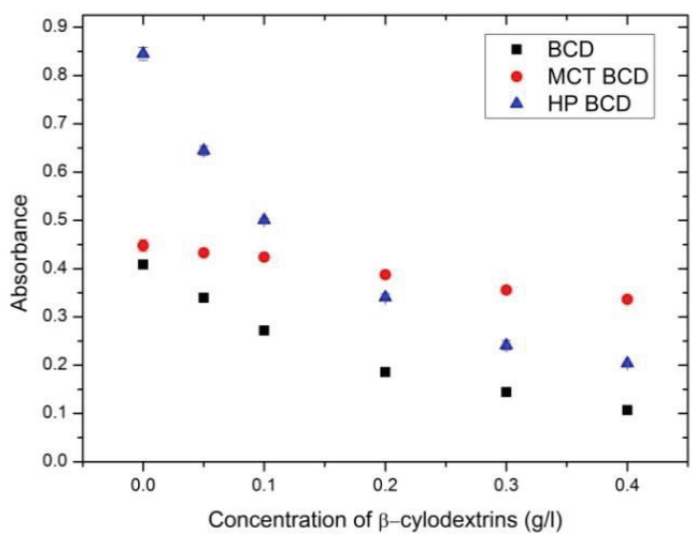

Figure 7.2: Calibration curves of BCD, HP BCD \& MCT BCD with phenolphthalein. From the calibration curves and the absorbances noted from phenolphthalein-buffer solutions with textile samples, the mass of $\beta$-cyclodextrin on the fabric could be estimated. The mass of the different $\beta$-cyclodextrins (BCD, HP BCD and MCT BCD) fixed on cotton $(\mathrm{F}, \mathrm{mg} / \mathrm{g})$ was calculated using the following equation:

$F\left(\frac{m g}{g}\right)=\frac{C \cdot V \cdot 1000}{M}$ 
where $\mathrm{C}$ refers to the concentration of $\beta$-cyclodextrin measured in $\mathrm{g} / \mathrm{l}, \mathrm{V}$ is volume in 1, M refers to the dry weight of fabric sample in $\mathrm{g}$.

The determination of BCD fixation amount from this method assumes that all of BCD cavities fixed on the fabric are accessible to the phenolphthalein molecules.

\subsubsection{The influence of BTCA on the Phenolphthalein tests}

A slight instability in phenolphthalein UV vis absorbance measurements was observed during the phenolphthalein tests described above in section 7.3.2. This was observed while measuring the absorbance of phenolphthalein solutions containing BCD and HP BCD fixed cotton samples. The instability in readings (characterized by repeated rapid rise and fall in absorbance) was particularly enhanced for fabrics fixed with only BTCA (control samples) or with fabrics fixed with lower amounts of BCD or HP BCD. This was attributed to the acidity of the polycarboxylic acid crosslinker (BTCA) used for the fixation of the respective $\beta$-cyclodextrin to the textile, while the phenolphthalein working solution used for the quantification is an alkaline one. This acidic crosslinker is fixed on the fabric in its free or unreacted form apart from its esterified form. This unreacted acid bound to the fabric does not refer to the unreacted BTCA which is removed during the rinsing step.

In order to observe the influence of BTCA on the phenolphthalein method of quantification of $\mathrm{BCD} / \mathrm{HP} \mathrm{BCD}$ on the textile, a simple test was conducted with the calibration curve described earlier in section 7.3.2. A $0.04 \mathrm{mM}$ solution of phenolphthalein was made and divided into two beakers. In one beaker, $1 \mathrm{~g} / 1 \mathrm{of}$ BTCA was added. Then two sets of test tubes were arranged, one set containing the 4 $\mathrm{ml}$ of phenolphthalein solution with BTCA and the other without the BTCA. $1 \mathrm{ml}$ of BCD solutions of various concentrations made in Tris (of $0.05 \mathrm{M}$ concentration) were then added to all the test tubes and the absorbances were then measured at $550 \mathrm{~nm}$ with Cary 100 UV spectrophotometer. The exact amount of unreacted acid on fixed on the fabric was unknown and therefore an arbitrary amount of BTCA was used in the experiment.

The results of the homogenous tests done with phenolphthalein, BCD and BTCA can be seen in Figure 7.3. Here the absorbance (A) of phenolphthalein is shown against 
concentration of BCD $(\mathrm{g} / \mathrm{l})$ in the presence and absence of BTCA. It can be seen that in the presence of BTCA, the absorbance values of the phenolphthalein solutions increase slightly. However, this increase in absorbance is corrected when the concentration of the BCD increases in the bulk. That is, the phenolphthalein absorbances values (with and without BTCA) overlap one another with the increasing amounts of $\mathrm{BCD}$ in the solution.

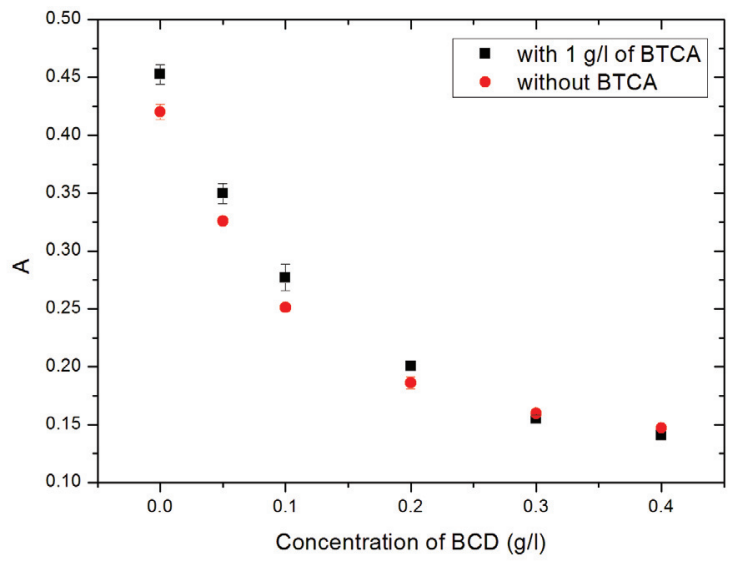

Figure 7.3: Influence of BTCA on the absorbance (A) of phenolphthalein solutions in presence of $\mathrm{BCD}$. All the measurements are an average of three readings.

This implied that it is likely that the amount of BCD fixed on the samples is underestimated by a small degree for fabrics treated at lower BCD bulk concentrations. However, since it appeared that this error was resolved in the presence of higher concentrations of $\mathrm{BCD}$, it was ignored. As mentioned earlier, correspondingly the absorbance readings of phenolphthalein solutions containing the BCD fabrics treated at higher bulk concentrations were stable.
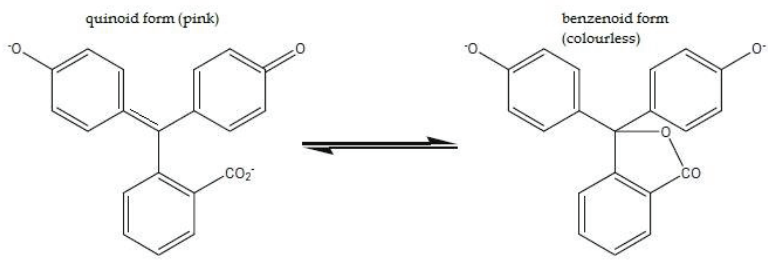

Figure 7.4: The quinoid and the benzenoid form of the phenolphthalein molecule 
As explained in chapter 6, the phenolphthalein molecule can exists in its benzenoid or quinoid form depending on the $\mathrm{pH}$ of the solution. There is an equilibrium between these two forms in the above alkaline solution (shown in Figure 7.4). This equilibrium is altered in the presence of the free acid groups attached to the fabric which leads to an instability in the UV vis absorbance readings. The pink colour of the alkaline phenolphthalein when in its quinoid form is due to the central carbon atom of the molecule which is conjugated in alkaline $\mathrm{pH}$ turning the molecule into a chromophore. However, with the complexation of such a phenolphthalein molecule into a BCD cavity, the molecule turns colourless due to the loss of this double bond in the central carbon atom (shown in Figure 6.6 of chapter 6). The instability in the UV vis absorbance is consequently resolved when an increased number of phenolphthalein molecules are in this state due to their complexation in the BCD cavity. The results of this work however do not say anything regarding the steric influence of the crosslinker attached BCD on the phenolphthalein absorbance.

The observations from this section can also be used to derive an additional conclusion over the calibration curve presented in Figure 7.2. In Figure 7.2 it can be seen that the phenolphthalein absorbance is influenced by the type of $\beta$-cyclodextrin molecule. This is despite the cavity size being the same in all the three types of $\beta$ cyclodextrin. This would therefore imply that the groups in the respective derivatives have an influence on the either the accessibility of the phenolphthalein molecule or on the equilibrium between the two forms of phenolphthalein. This would therefore also mean that the crosslinked BTCA might have a steric influence on the phenolphthalein absorbance when complexed within the BCD cavity (fixed on the textile). Studies investigating the steric influence of the crosslinkers on $\beta$ cyclodextrins complexed with phenolphthalein are required to conclude further.

\subsection{Characterization tests of BCD treated textiles}

Characterization tests were done on BCD fixed cotton using the Fourier Transform Infra Red-Attuended Total Reflectance spectroscopy (FTIR-ATR) and the Differential Scanning Calorimetry (DSC) measurements. The results of the $\beta$-cyclodextrins fixation experiments had showed that $B C D$ was the most best suited and therefore these characterization experiments were done only on BCD fixed cotton. 


\subsubsection{FTIR-ATR spectroscopy measurements}

\subsubsection{Background}

FTIR spectroscopy is a technique through which specific functional groups present on a substrate can be identified due to their fingerprint infrared absorption. Figure 7.5 shows the main infrared wavenumbers and the respective functional groups measured with FTIR spectroscopy.

As mentioned earlier, the esterification reaction of cotton with BTCA creates ester and bound free acid groups in the fabric. The infrared spectrum obtained by making FTIR spectroscopic measurements of these fabrics show peaks relating to these functional groups.

\begin{tabular}{|c|c|c|}
\hline Wave number, $\mathrm{cm}^{-1}$ & Functional Group & Peak Description \\
\hline $3300-3600$ & $\mathrm{O}-\mathrm{H}$ (alcohol) & Strong and broad \\
\hline $\begin{array}{l}2500-3000 \\
\text { can reach }\end{array}$ & $\mathrm{O}-\mathrm{H}$ (carboxylic acids) & $\begin{array}{l}\text { Very broad (over } \sim 500 \mathrm{~cm}^{-1} \text { ), often } \\
\text { looks like distorted baseline, } \\
\text { above } 3000 \mathrm{~cm}^{-1}\end{array}$ \\
\hline $3200-3500$ & $\mathrm{~N}-\mathrm{H}$ & $\begin{array}{r}\text { Doublet in case of } \mathrm{NH}_{2} \text { group of } \\
\text { a primary amine or amide }\end{array}$ \\
\hline 3300 & $\begin{array}{l}\equiv \mathrm{C}-\mathrm{H} \\
\text { terminal alkyne }\end{array}$ & Usually sharp and strong \\
\hline $3000-3100$ & $=\mathrm{C}-\mathrm{H}$ & $\begin{array}{l}\text { Often weak, overlaps with } \mathrm{CH} \text { alkane } \\
\text { absorption }\end{array}$ \\
\hline $2800-3000$ & $\mathrm{C}-\mathrm{H}\left(\mathrm{sp}^{3}\right.$ carbon) & Strong, broad and multi-banded \\
\hline $2250-2220$ & $\mathrm{C} \equiv \mathrm{N}$ & Medium intensity \\
\hline $2100-2260$ & $\begin{array}{l}\mathrm{C} \equiv \mathrm{C} \\
\text { alkyne }\end{array}$ & $\begin{array}{l}\text { Medium intensity for terminal } \\
\text { alkynes, very weak for internal }\end{array}$ \\
\hline $1680-1820$ & $\begin{array}{l}\quad \mathrm{C}=\mathrm{O} \\
\text { (amides, ketones, aldehydes } \\
\text { carboxylic acid, esters) }\end{array}$ & $\begin{array}{l}\text { Very strong; } \\
\text { lower frequency for amides and } \\
\text { when } \mathrm{C}=\mathrm{O} \text { is conjugated }\end{array}$ \\
\hline $1600-1650$ & $\begin{array}{l}\mathrm{C}=\mathrm{C} \\
\text { alkene, aromatic ring }\end{array}$ & $\begin{array}{l}\text { Check to see if you have C-H } \\
\text { unsaturated }>3000 \mathrm{~cm}^{-1} \text { (if not, it's } \\
\text { completely substituted) }\end{array}$ \\
\hline$\sim 1600$ & $\begin{array}{l}-\mathrm{NH}_{2} \text { (bending) } \\
1^{\circ} \text { amines and amides }\end{array}$ & $\begin{array}{l}\text { Only if you have corresponding } \mathrm{N}-\mathrm{H} \\
\text { peak at } 3200-3500 \mathrm{~cm}^{-1} \text { (this peak } \\
\text { may be mistaken for } \mathrm{C}=\mathrm{C} \text { otherwise) }\end{array}$ \\
\hline 1200 & Ar-O & Strong (look for $=\mathrm{C}-\mathrm{H} \& \mathrm{C}=\mathrm{C}$ first) \\
\hline $1050-1150$ & $\mathrm{C}-\mathrm{O}$ & \\
\hline 690 and 750 & phenyl group & Strong (look for $=\mathrm{C}-\mathrm{H} \& \mathrm{C}=\mathrm{C}$ first) \\
\hline
\end{tabular}

Figure 7.5: Table of main IR wavenumber and the respective functional groups [22]. 
The peak intensities of these functional groups can then be used to do semi quantitative analysis of the BTCA crosslinking effectiveness on cotton. In our work, these spectroscopy measurements were done to assess the influence of BCD on the formation of ester on cotton with BTCA.

\subsubsection{FTIR analysis of BTCA crosslinked cotton}

In literature, the use of FTIR spectroscopy to analyze BTCA crosslinked cotton is described $[4,13,16,23-26]$. In order to acquire the respective peaks of various functional groups on the BTCA treated fabrics, the FTIR analysis method requires a preparation step. The samples need to be pretreated with sodium hydroxide $(\mathrm{NaOH})$ or ammonia vapour before the analysis. The reason for this is that a cotton fabric esterified with BTCA, has three types of carbonyl groups $(C=O)$ on its surface. One type of the carbonyl group belongs to the carboxyl acid fixed to the cotton and the other belongs to the ester fixed to the cotton. The third belongs to the carbonyl groups on the fabric in their carboxylate anion form. This later type of carbonyls can be noted at $1570 \mathrm{~cm}^{-1}$. The carbonyls of the former two types are however not differentiated by the FTIR instrument. These two types of carbonyls shown up under one peak at $1720 \mathrm{~cm}^{-1}$. By pretreating the fabric with $\mathrm{NaOH}$ or ammonia vapour, these two types of carbonyl groups can be separated. The treatment with $\mathrm{NaOH}$ converts the carbonyls belonging to the acid to the carboxylate anion form and this can be seen by the increase in the peak at $1570 \mathrm{~cm}^{-1}$ and an decrease in the peak at $1720 \mathrm{~cm}^{-1}$. By exposing this pretreated fabric to hydrogen chloride $(\mathrm{HCl})$ vapour, this separation of carbonyls can be reversed. This happens due to the protonation of ionized carboxylate groups (COO-to $\mathrm{COOH}$ ) on cotton. This consequently shows up as an increased peak at $1720 \mathrm{~cm}^{-1}$ in the FTIR spectroscopic analysis [27].

After the pretreatment, the intensity at $1720 \mathrm{~cm}^{-1}$ peak now shows only the total ester on the fabric i.e. the ester bonds within the cotton and the covalent ester bonds formed after the esterification reaction between cotton and BTCA (in case of only BTCA treated fabrics). The ratio between the ratio of the intensities at $1720 \mathrm{~cm}^{-1}$ to $1570 \mathrm{~cm}^{-1}$ can be used to calculate the crosslinking effectiveness of BTCA in the treated cotton $[5,24,28]$. This ratio is referred to as the degree of esterification. It indicates the average number of ester groups formed per BTCA molecule fixed on 
cotton and therefore is a relative measure of the effectiveness of BTCA as a crosslinking agent. These values can be used to correlate to other properties of the textile that are influenced by BTCA crosslinking such as the wrinkle recovery angle and tensile strength of a fabric [14].

It is known that with an increase in curing temperature or curing time, an increase in the ester carbonyl band intensity ratio is observed indicating that an increase in the number of ester groups are formed per BTCA molecule [5, 29]. However the literature is unclear on the influence of BTCA concentration on the degree of crosslinking. Some literature report that an increase in BTCA concentration leads to increase in crosslinking and this can be observed with the increased FTIR ester carbonly intensity ratio $[16,23,28]$. This is contradicted by other literature which report that although the total amount of ester on the fabric increases with an increase in the BTCA concentration, the crosslinking effectiveness of BTCA reduces and this is due to limited hydroxyl groups available on cotton $[29,30]$.

It has been reported that the $\mathrm{NaOH}$ method of sample preparation is suitable only for the assessment of degree of esterification on BTCA treated cotton and not for cotton crosslinked with other polycarboxylic acids [23]. In our work, we also used the ammonia vapour method of sample preparation. However, we found that these two methods were not comparable. The ammonia vapour method gave irregular readings and this could have been due to the complexation of ammonia vapour inside the BCD cavity.

\subsubsection{FTIR-ATR analysis in this work}

For this work, FTIR-ATR spectroscopy was used instead of FTIR spectroscopy. This is a non-destructive method of sample analysis in comparison with the general FTIR spectroscopy analysis. The $\mathrm{NaOH}$ preparation step for this FTIR-ATR analysis involved treating the cotton fabric samples with $0.1 \mathrm{M} \mathrm{NaOH}$ for a minimum of 3 minutes, washing off the $\mathrm{NaOH}$ from the fabric and then drying the textile sample at $90{ }^{\circ} \mathrm{C}$ for 10 minutes before the FTIR-ATR spectroscopy measurement.

We scanned all the different samples treated with BCD and BTCA. The variables were BCD concentration (0-100 g/l) and BTCA concentration (30 \& $60 \mathrm{~g} / \mathrm{l})$. Further, 
the influence of curing was also investigated on the formation of ester on the fabric. The ester intensity was observed for all fabrics at the earlier mentioned wave number of $1720 \mathrm{~cm}^{-1}$. The peak intensity at $1570 \mathrm{~cm}^{-1}$ was not considered. The reasons for this were that the degree of esterification calculations give a relative measure of the crosslinking effectiveness of BTCA in only BTCA treated cotton, here however we have two reactants in the system (BCD and BTCA). Secondly, the ester linkages are relatively homogenously spread within the fabric while the carboxylates have higher concentration on the outer surface rather than its interior [24]. The FTIR-ATR spectroscopy is a near surface analysis technique and use of the carboxylate intensity for degree of esterification calculations would consequently lead to erroneous results.

FTIR-ATR spectra were obtained on a Bruker IFS 66/S spectrophotometer, equipped with Silver gate ATR cell employing a cylindrical Ge crystal $(n=4.0)$ with a diameter of $7 \mathrm{~mm}$ and single reflection (SpectraTech). The samples were tightly pressed against a Ge crystal and the spectra were recorded over the range 4.000-600 $\mathrm{cm}^{-1}$, with a resolution of $4 \mathrm{~cm}^{-1}$ and averaged over 128 spectra. For all the spectra generated a normalization was done at $1313 \mathrm{~cm}^{-1}$.

\subsubsection{Differential scanning calorimetry measurements}

As mentioned earlier, BTCA is known to give flame resistant properties to cotton. The use of this crosslinker was also desirable due to this additional functionality. One of the simplest way of observing the influence of flame retardant molecules on any surface is by doing a DSC measurement of the samples. In this thermoanalytical test, the differences in the heat required to increase the temperature of a sample as compared to a blank sample is measured.

Differential scanning calorimetry (DSC) measurements were done to check the influence of BCD and BTCA treatment on thermal decomposition of cotton. The cotton samples assessed were treated with $10 \mathrm{~g} / 1,30 \mathrm{~g} / 1$ and $60 \mathrm{~g} / 1$ of BTCA with 0 $\mathrm{g} / \mathrm{l}$ and $100 \mathrm{~g} / 1$ of BCD at various curing conditions. These measurements were done under nitrogen flow of $20 \mathrm{ml} / \mathrm{min}$. The samples were first held isothermally for a minute at $25^{\circ} \mathrm{C}$ and then heated to $550{ }^{\circ} \mathrm{C}$ at the rate of $10^{\circ} \mathrm{C} / \mathrm{min}$. The cooling was done at the rate of $60^{\circ} \mathrm{C} / \mathrm{min}$. Sample weights for these measurements were around 9.3 to $9.8 \mathrm{mg}$. 


\subsection{Laundering tests}

Laundering tests were done on samples treated with the three $\beta$-cyclodextrin types. The aim of these tests was to observe the washing fastness of the samples. Cotton samples treated with BCD, HP BCD and MCT BCD at $100 \mathrm{~g} / 1$ application concentration were used for these tests since these samples showed the highest amount of $\beta$-cyclodextrin fixation. These samples were going to be used for further applications. BCD and HP BCD crosslinking with cotton was done with $30 \mathrm{~g} / 1$ of BTCA (1:0.3 molar ratio of BTCA to SHPI). The curing was done at $160^{\circ} \mathrm{C}$ for 5 minutes for all the samples as mentioned earlier. These samples were laundered with $4 \mathrm{~g} / 1$ of a ECE reference detergent (without optical brighteners, details described in chapter 4). The laundering was done at an LCR of $50: 1$ at $60^{\circ} \mathrm{C}$ for 30 minutes. After the laundering, the samples were rinsed in tap water and then dried in the oven for 15 minutes at $110{ }^{\circ} \mathrm{C}$. The estimation of the amount of $\beta$-cyclodextrin remaining on the samples was done (as described in section 7.3.2) after the washing cycles.

\subsection{Results and discussion}

\subsubsection{Quantification of cyclodextrins fixed on cotton}

\subsubsection{Gravimetric measurements}

The results of the gravimetric measurements are shown as weight gain (W\%) vs. application or bulk concentrations of the various $\beta$-cyclodextrins in Figures 7.6a \& 7.6b. Figure 7.6a shows the weight gain for the different treatments conditions; i.e three different concentrations of BTCA and the various BCD bulk concentrations. Figure $7.6 \mathrm{~b}$ shows the results obtained for the treatments with all the three types of $\beta$ cyclodextrins used (BCD, HP BCD, MCT BCD). It is obvious from Figure 7.6a that a steady increase of weight gain $\mathrm{W} \%$ is observed with the increase in the BCD bulk concentration. However, the crosslinker BTCA contributes significantly to the weight gain values as compared to the weight gain of the fixed $\mathrm{BCD}$. The difference between the weight of samples treated with BCD and without BCD cannot be used since this method appears to be prone to significant error. 


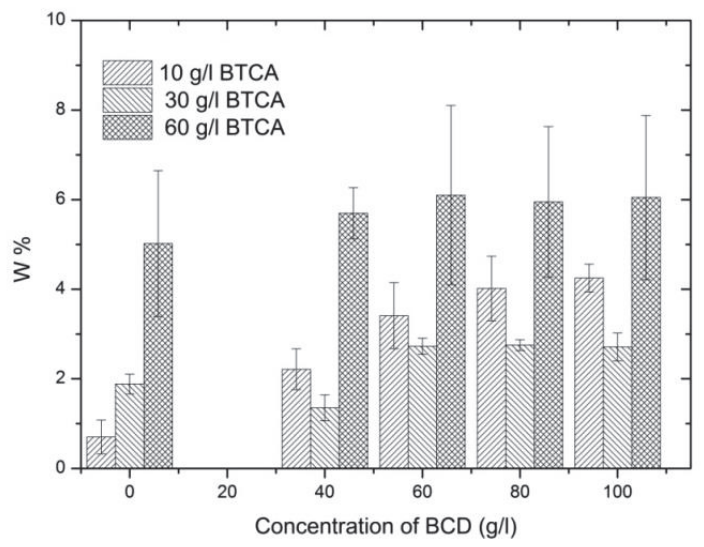

Figure 7.6a: Weight gain (W\%) of cotton treated with varying applied concentrations of BCD for $10 \mathrm{~g} / \mathrm{l}, 30 \mathrm{~g} / 1$ and $60 \mathrm{~g} / 1$ of BTCA concentrations. All the measurements are an average of three samples.

In Figure 7.6b a comparison of weight gain is made between BCD, HP BCD (both fixed with $30 \mathrm{~g} / 1$ BTCA) and MCT BCD treated fabrics. The influence of increasing $\beta$ cyclodextrin concentration on the weight gain is seen only for cotton treated with BCD (already shown also in Figure 7.6a) and with MCT BCD.

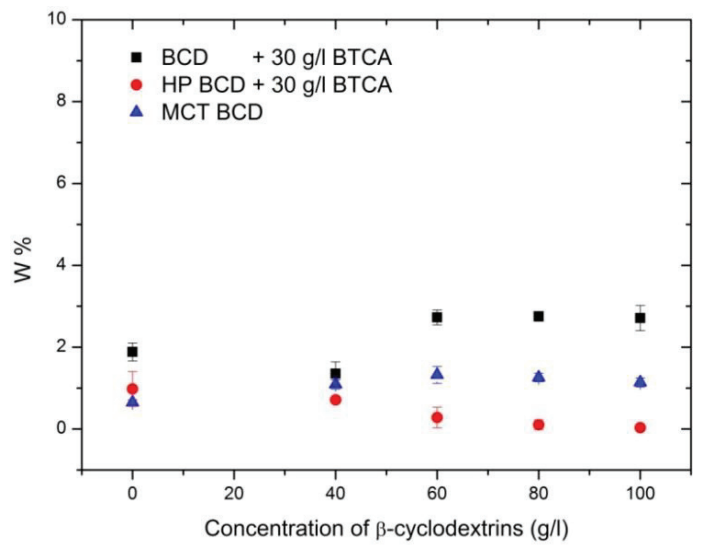

Figure 7.6b: Weight gain (W\%) of cotton treated with varying concentrations and types of $\beta$-cyclodextrin. BCD and HP BCD were fixed to cotton with $30 \mathrm{~g} / 1$ of BTCA, while MCT BCD was attached directly to cotton. All the measurements are an average of three samples. 
HP BCD shows a slight loss of the weight gain W\% with increasing concentration. These results indicate that weight gain measurements do not correspond to actual fixation amount.

\subsubsection{Phenolphthalein method}

The amount of $\beta$-cyclodextrin fixed on the cotton assessed by the phenolphthalein method is shown in Figure 7.7a and 7.7b as F (mg/g) against the applied or bulk concentrations of $\beta$-cyclodextrin (g/1). Figure 7.7a shows the amount of BCD fixed (in $\mathrm{mg}$ ) per gram of cotton with varying concentrations of BTCA. From these results it appears that the crosslinker concentration has no significant influence on the BCD fixation amount. Among the three selected crosslinker concentrations, more repeatable values are seen for BCD cotton treated with $30 \mathrm{~g} / 1$ of BTCA. Therefore this concentration of the crosslinker was selected for all further BCD fixation experiments.

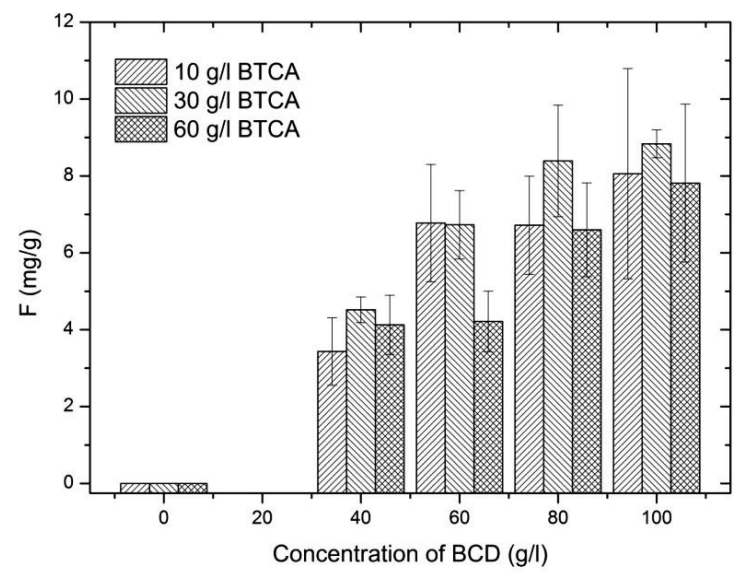

Figure 7.7a: Amount of BCD fixed on cotton with varying applied concentrations of $\mathrm{BCD}$ and $10 \mathrm{~g} / \mathrm{l}, 30 \mathrm{~g} / 1$ and $60 \mathrm{~g} / 1$ of BTCA. All the measurements are an average of three samples.

Curing conditions were then changed to observe their influence on the fixation of $\mathrm{BCD}$ on cotton. For these experiments, amount of BCD bulk concentration was kept at $100 \mathrm{~g} / 1$ while the temperature, catalyst concentration and amount of BTCA were changed as shown in Table 7.1. 
Treatment conditions

Bath concentration of BTCA Curing temperature $\left(^{\circ}\right.$ $(\mathrm{g} / \mathrm{l})$ C)

60

160

BTCA to catalyst ratio (molar ratio)

$F$ $(\mathrm{mg} / \mathrm{g})$

60

$60 \quad 180$

30
30

160

180

180
1:0.3

1:0.3

$1: 1$

1:0.3

1:0.3

$1: 1$
7.82

7.45

5.95

8.83

9.10

8.91

Table 7.1: Amount of unmodified BCD, F (mg/g) for different treatment and curing conditions. The BCD bulk concentration was kept at $100 \mathrm{~g} / 1$.

A significant increase in fixation by improvement in curing conditions was not observed as can be seen in the table. At $60 \mathrm{~g} / 1$ of BTCA concentration, at a BTCA to catalyst molar ratio of 1:1 and high curing temperature, the amount of BCD fixation in fact reduced.

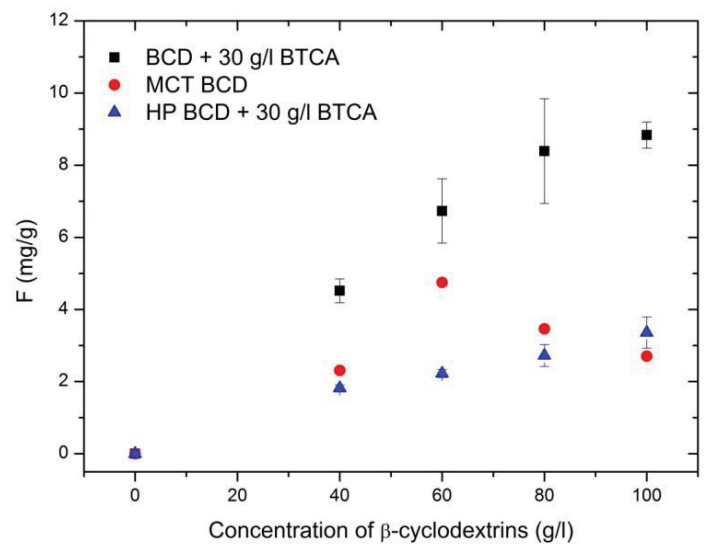

Figure 7.7b: Amount of $\beta$-cyclodextrins fixed on cotton. BCD and HP BCD are fixed with $30 \mathrm{~g} / 1$ of BTCA while MCT BCD is attached directly. All the measurements are an average of three samples.

The results of the fixation experiments done with the different types of $\beta$ cyclodextrins is shown in Figure 7.7b. It can be seen that the amount of fixation is highest for the $\mathrm{BCD}$ or the unmodified type of $\beta$-cyclodextrin. The amount of fixation of the three $\beta$-cyclodextrins on cotton is also shown in moles per gram in Table 7.2. 


\begin{tabular}{|c|c|c|c|}
\hline $\begin{array}{c}\text { Application } \\
\text { concentration }(\mathrm{g} / \mathbf{l})\end{array}$ & $\begin{array}{c}\text { BCD with } 30 \mathrm{~g} / \mathbf{l} \text { of } \\
\text { BTCA (moles/g) }\end{array}$ & $\begin{array}{c}\text { HP BCD with } 30 \mathrm{~g} / \mathbf{l} \text { of } \\
\text { BTCA (moles/g) }\end{array}$ & $\begin{array}{c}\text { MCT BCD } \\
\text { (moles/g) }\end{array}$ \\
\hline 0 & 0 & 0 & 0 \\
\hline 40 & $3.98 \times 10^{-6}$ & $1.29 \times 10^{-6}$ & $1.47 \times 10^{-6}$ \\
\hline 60 & $5.93 \times 10^{-6}$ & $1.59 \times 10^{-6}$ & $3.04 \times 10^{-6}$ \\
\hline 80 & $7.39 \times 10^{-6}$ & $1.94 \times 10^{-6}$ & $2.21 \times 10^{-6}$ \\
\hline 100 & $7.78 \times 10^{-6}$ & $2.40 \times 10^{-6}$ & $1.73 \times 10^{-6}$ \\
\hline
\end{tabular}

Table 7.2: Calculated amount of fixed $\beta$-cyclodextrin in moles per $\mathrm{g}$ of cotton.

The lower amount of fixation of HP BCD as compared to BCD on cotton for the same fixation conditions was unexpected since the solubility of HP BCD in water is much higher than BCD. However the results obtained can be explained by considering the fact that $\mathrm{HP}$ BCD is a derivative with long 2- hydroxypropyl groups in place of hydroxyl groups in the anhydroglucopyranose units of the BCD molecule. From the supplier of this HP BCD, it is also known that an average of 5.6 hydroxypropyl groups are substituted per BCD molecule in this derivative. This therefore leaves fewer free hydroxyl groups to participate in the esterification reaction. This is especially more so for the hydroxyl groups at C-6 position which are more inclined to participate in the esterification reaction [31]. The hydroxyl groups in the hydroxypropyl chains are less likely to participate in this esterification reaction since they are not easily accessible being sandwiched between the alkyl groups. This can be seen in Figure 7.7c taken from the brochure given by the supplier.

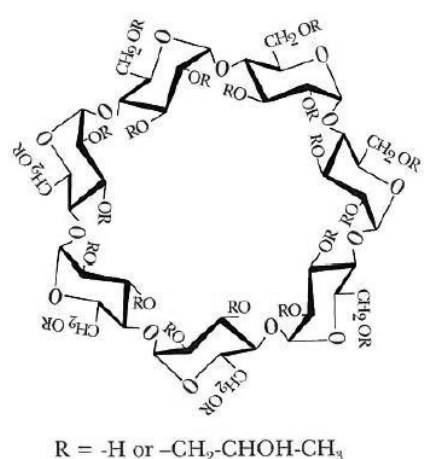

Figure 7.7c: Structure of HP BCD[1].

Further the increased solubility of HP BCD as compared to BCD is in aqueous solution at a $\mathrm{pH}$ of 7 . However, the fixation experiments were done in acidic $\mathrm{pH}$ (due 
to the addition of BTCA). This acidic bulk liquor increased the solubility of BCD significantly as mentioned earlier. Therefore the difference in aqueous solubility these two $\beta$-cyclodextrins in neutral $\mathrm{pH}$ did not have an influence on the fixation amount in these experimental conditions.

The lower fixation amount of MCT BCD can be expected due to the reason that this derivative contains 2-3 reactive monochlorotriazinyl group per BCD molecule. This is much lesser than the free hydroxyl groups available for esterification with the unmodified molecule (BCD).

\subsubsection{FTIR-ATR spectroscopy measurements}

FTIR-ATR spectroscopic analysis was done as mentioned earlier in order to study the influence of BCD on the esterification of the cotton fabric with BTCA. BCD molecule also forms an ester bond with BTCA along with ester formed between cotton and BTCA as shown in Figure 7.1. Each BTCA molecule is capable of forming one to three ester groups with the available reactants. These are referred to as mono, di and triesters respectively.

The esterification reaction between cotton, BTCA and BCD leads to a mix of different ratios of mono, di and tri-esters. Among these different esters formed, mono-esters do not contribute to crosslinking within the cellulose network (i.e. this refers to cotton-BTCA or BCD-BTCA which is rinsed away). The linking of the BCD molecule to cotton requires the formation of atleast a di-ester (cotton-BTCA-BCD). In the current experimental curing conditions, a mixture of mono and di-esters was expected as tri-esters are reported to be predominant only at much higher curing temperatures $\left(200^{\circ} \mathrm{C}\right)$ [29]. The crosslinking network is therefore propagated by these di-esters via cotton substrate and the BCD molecule (example, BCD-BTCAcotton-BTCA-BCD and cotton-BTCA-BCD-BTCA-cotton) [9, 32].

Figure 7.8a shows the FTIR-ATR spectra of the cotton fabrics treated with different BCD bulk concentrations via a BTCA concentration of $30 \mathrm{~g} / 1$. A reduction in the ester peak intensity (at $1720 \mathrm{~cm}^{-1}$ ) and the carbonyl peak intensity (at $1580 \mathrm{~cm}^{-1}$ ) is seen when the BCD bulk concentration is increased. The same pattern was observed for 
fabrics treated with different BCD bulk concentrations via a BTCA concentration of $60 \mathrm{~g} / 1$.

The ester intensity values obtained from the different fabrics is shown in Table 7.3. Here it is seen that ester intensity is increased in fabrics treatments with $60 \mathrm{~g} / \mathrm{l}$ as compared to $30 \mathrm{~g} / 1$ of BTCA. Therefore a higher concentration of BTCA leads to an increase in the number of the ester bonds formed in the fabric. Since an increase in the crosslinker concentration does not influence the fixation amount of BCD to cotton (as described in section 7.6.1.2), it can concluded that the increase in ester bonds here is not contributed by ester linkages to BCD but only between the cellulose and BTCA.

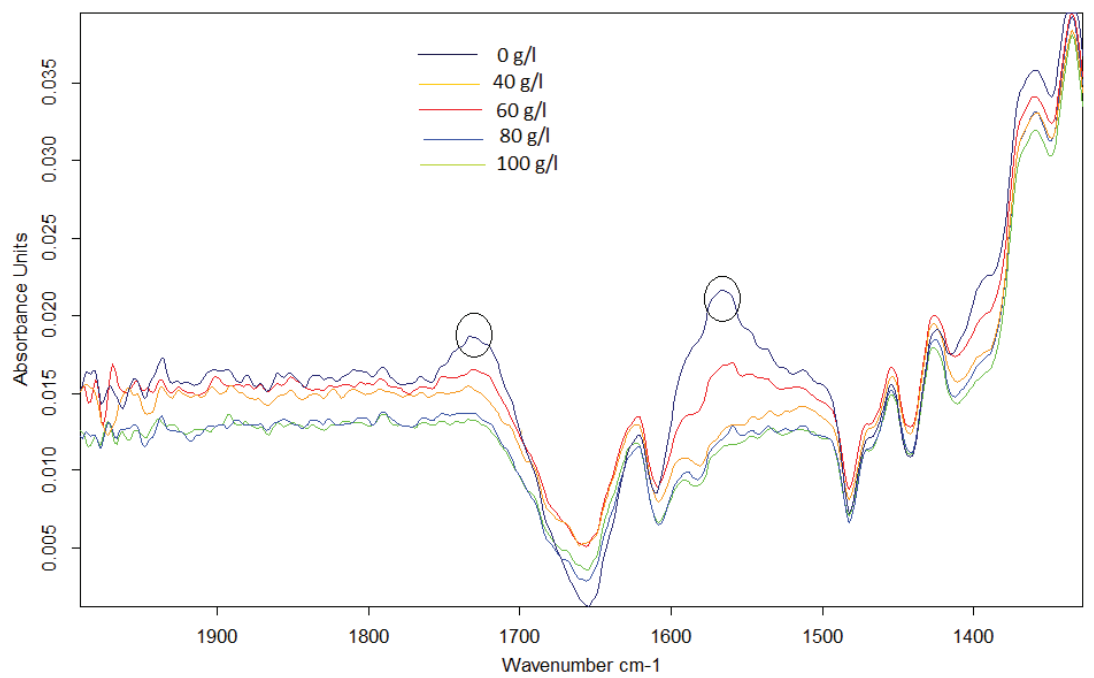

Figure 7.8a: FTIR-ATR spectra of cotton samples treated with different BCD bulk concentration via $30 \mathrm{~g} / \mathrm{l}$ of BTCA. The legend shows the BCD bulk concentration. The peaks at $1720 \mathrm{~cm}^{-1}$ and $1570 \mathrm{~cm}^{-1}$ are shown with a circle for one sample.

It also appears that this ester intensity reduces gradually with the increasing BCD bulk concentration for both the crosslinker concentrations. Further, the influence of the curing conditions on the ester intensity at $1720 \mathrm{~cm}^{-1}$ of cotton fabrics was also observed. 


\begin{tabular}{|c|c|c|c|}
\hline $\begin{array}{c}\text { Concentration of } \\
\text { BTCA }(\mathrm{g} / \mathbf{l})\end{array}$ & $\begin{array}{c}\text { Application concentration of } \\
\text { native BCD }(\mathrm{g} / \mathbf{l})\end{array}$ & $\begin{array}{c}\text { Ester peak } \\
\text { intensity }\left(\mathbf{c m}^{-1}\right)\end{array}$ & $\begin{array}{c}\text { Standard } \\
\text { deviation }\end{array}$ \\
\hline 30 & 0 & 0.372 & 0.029 \\
\hline 30 & 40 & 0.346 & 0.021 \\
\hline 30 & 60 & 0.334 & 0.022 \\
\hline 30 & 80 & 0.296 & 0.016 \\
\hline 30 & 100 & 0.291 & 0.013 \\
\hline 60 & 0 & 0.390 & 0.007 \\
\hline 60 & 40 & 0.393 & 0.058 \\
\hline 60 & 60 & 0.357 & 0.023 \\
\hline 60 & 80 & 0.351 & 0.057 \\
\hline
\end{tabular}

Table 7.3: The ester peak intensity $\left(1720 \mathrm{~cm}^{-1}\right)$ with different concentration of BTCA and increasing native BCD application concentration.

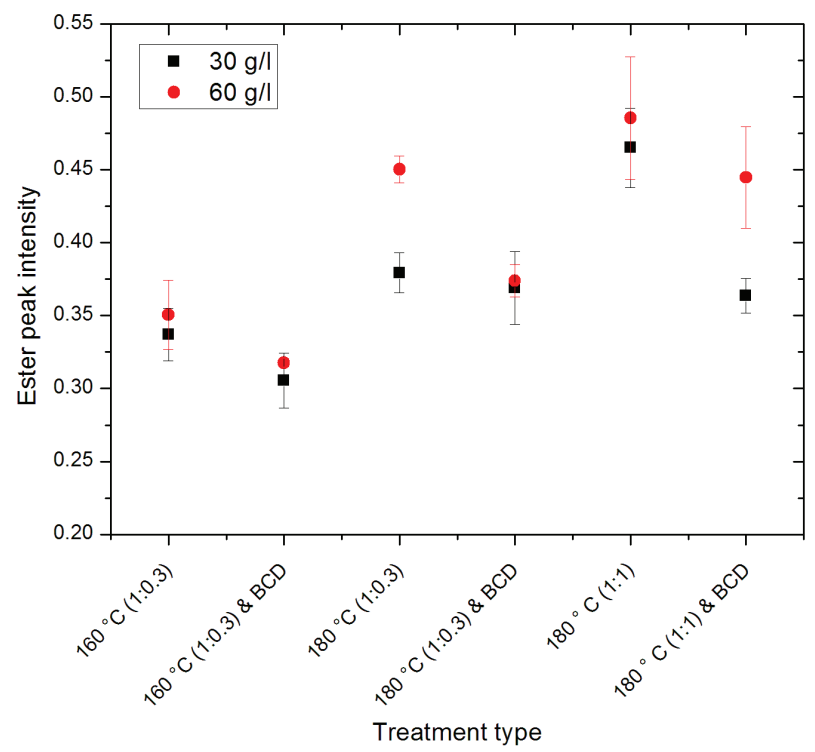

Figure 7.8b: Ester peak intensity at $1720 \mathrm{~cm}^{-1}$ (normalized at $1313 \mathrm{~cm}^{-1}$ ) with change in curing temperature and amount of BTCA to catalyst mole ratio shown in brackets. Amount of BCD applied was $100 \mathrm{~g} / 1$ and concentrations of BTCA used were $30 \mathrm{~g} / 1$ and $60 \mathrm{~g} / 1$. All readings were an average of three samples.

In Figure $7.8 \mathrm{~b}$, it can be seen that the total amount of the covalent ester formed on the fabric increases with the curing temperature or with an increase in the BTCA to 
catalyst ratio as expected. However, with the addition of BCD in each case, this peak intensity is again seen to reduce.

These observations lead to the conclusion that though some BCD does takes part in the esterification reaction, the reaction is not as effective as when compared to the reaction between only BTCA and cotton.

As explained earlier, an anhydride intermediate is formed prior to the formation of an ester in the esterification reaction. It is possible that the conversion of the anhydride to the ester is hindered due to some reason. This can be easily detected in the FTIR-ATR spectrum by observing the anhydride intensities. The anhydride intensities can be observed at $1782 \mathrm{~cm}^{-1}$ and $1852 \mathrm{~cm}^{-1}$. These measurements showed that with increasing BCD bulk concentration, the intensities reduced for $30 \mathrm{~g} / 1$ of BTCA and the trend was mixed with $60 \mathrm{~g} / 1$ of BTCA (shown in Table 7.4). In case the anhydride intensities would have increased while ester intensities reduced, it could have been concluded that the lower esterification was due to the lower mobility of the anhydrides intermediates towards the cellulosic hydroxyl groups. This is known to happen if the molecular weight of an polycarboxylic acid is quite large [4]. However since this was not the case, it was concluded that this reduction in the formation of ester has to do prior to the formation of the anhydride intermediate.

It is known that the reactivity of carboxylic acids and other compounds such as alcohols towards esterification are limited by steric hindrances of the carboxylic acid and of the other reactants in the system [33]. It has also been said that steric hindrances in BCD can have an influence on its grafting on to a cellulosic surface [34]. The reduction in ester formation in presence of BCD is attributed here to the steric hindrance that the large BCD molecule presents leading to the reduced reactivity between the various reagents in the given experimental conditions.

\subsubsection{Differential scanning calorimetry measurements}

The pyrolysis of cotton initiates a hemolytic chain scission within the cellulose structure leading to the formation of an intermediate pyrolysis product called levoglucosan (shown in Figure 7.9a). The reactivity of hydroxyl group at the C-6 
position of the anhydroglucopyranose unit of the cellulose chain is crucial in this depolymerization reaction.

According to $\mathrm{Wu}$ et.al [35], esterification of cotton via a treatment with BTCA can give flame retardant properties to the fabric due to the reason that the esterification reaction involves the hydroxyl groups at the C-6 position of cotton's glucopyranose unit. Ester formation at the C-6 position therefore blocks this site and retards the levoglucosan formation by reducing the depolymerization of cotton during the pyrolysis [36]. Further, the esterification reaction due to a tetracarboxylic acid also leads to an increase in the char content of the fabric due to the increase in carboncarbon network.

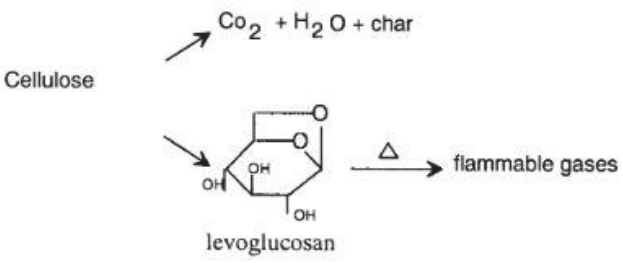

Figure 7.9a: Pyrolysis of cotton [36].

This as well as the additional bonds provided by the crosslinking increases the fabric's thermal stability. It is reported that the reduction in flammability is also significantly influenced by the presence of the phosphorous catalyst used during the esterification of BTCA to cotton [7].

The DSC spectra shows the changes in the heat released or heat absorbed by the material as against the temperature of the material. In Figure 7.9b, a small exothermic peak is observed at $170{ }^{\circ} \mathrm{C}$ for untreated cotton and cotton treated with $10 \mathrm{~g} / \mathrm{lof}$ BTCA. This is also observed in samples treated with BCD (via BTCA) in Figure 7.9c. Samples treated with only BTCA ( $30 \mathrm{~g} / 1 \& 60 \mathrm{~g} / \mathrm{l})$ do not show these peaks. These exothermic peaks are due to the crosslinked BTCA on the surface of cotton. 


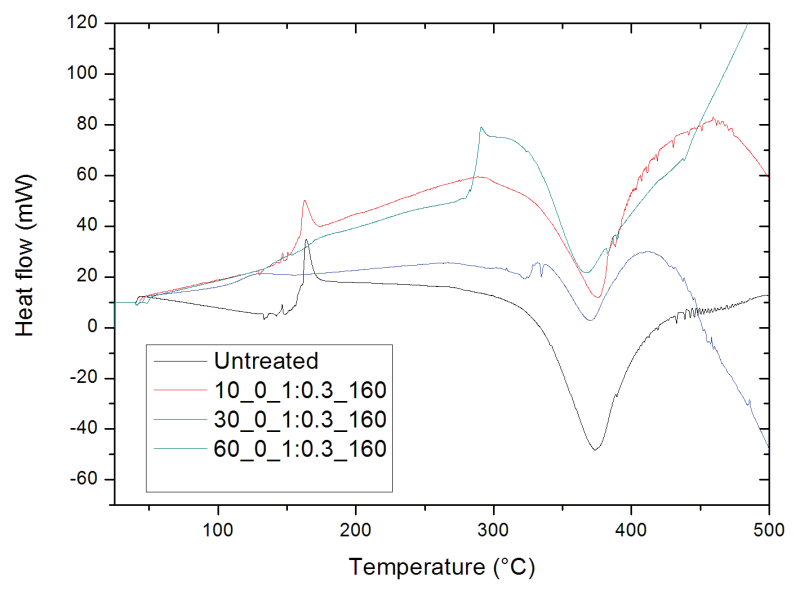

Figure 7.9b: DSC figures of treated and untreated cotton. The legend describes first the concentration of BTCA $(\mathrm{g} / \mathrm{l})$, followed by concentration of BCD $(\mathrm{g} / \mathrm{l})$, this is followed by the mole ratio of BTCA to catalyst and finally the curing temperature $\left({ }^{\circ} \mathrm{C}\right)$.

The untreated cotton sample shows an endothermic peak around $370{ }^{\circ} \mathrm{C}$. This endothermic peak is characteristic of the thermal decomposition of cellulose [37]. It is a result of the depolymerization of the cellulose leading to the formation and evaporation of levoglucosan. DSC measurements in literature show this thermal decomposition temperature to be significantly lowered in case of flame retardant samples due to the effluence of the flame retardants [38, 39]. In some cases, this endothermic peak is entirely absent. In this work, no significant differences were noted in this decomposition temperature between the DSC curves of the various treated and the blank cotton samples. Additional tests done with Microscale Combustion Calorimeter (MCC) also did not show any significant differences between the various treatments. The absence of any significant difference in the thermal behavior of the treated samples in this work is considered to be due to the use of low amount of the phosphorous based catalyst. Further experimental work is required to understand the impact of the catalyst and BTCA curing conditions on the flame retardancy of BTCA and BCD treated cotton. 


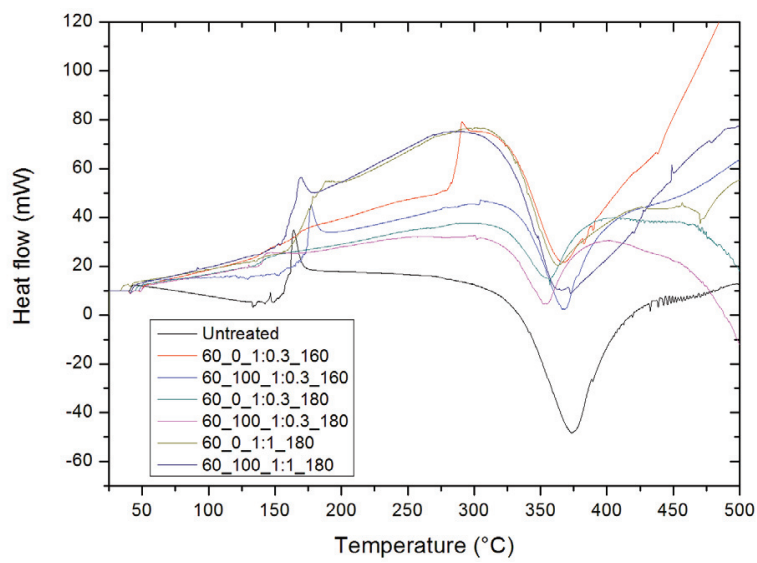

Figure 7.9c: DSC figure of cotton treated with and without BCD at various curing conditions. The legend describes first the concentration of BTCA $(\mathrm{g} / \mathrm{l})$, followed by concentration of $\mathrm{BCD}(\mathrm{g} / \mathrm{l})$, this is followed by the mole ratio of BTCA to catalyst and finally the curing temperature $\left({ }^{\circ} \mathrm{C}\right)$.

\subsubsection{Laundering tests}

The results of the laundering tests showed that the $\beta$-cyclodextrins fixed on the cotton surface were not wash durable (shown in Figure 7.10). The low wash fastness of BCD and HP BCD is an indication of poor wash fastness of the ester bonds that link them to cotton. However it is known that treatment of BTCA on to cotton is wash durable (due to its use in crease resistant recovery finishes).

As explained earlier, each BTCA molecule is capable of producing one to three ester bonds. In the current curing conditions, a mix of one to two ester bonds were expected for each BTCA molecule within the fabric. Each BCD molecule requires atleast one ester bond for its fixation to cotton. In the absence of the BCD molecule, this ester bond is used to crosslink the cellulose chains within the cotton structure. In presence of a $\mathrm{BCD}$ molecule, the crosslinking to cotton is reduced due to the deviation of one ester linkage away from the cellulose in order to link the BCD molecule (cotton-BTCA-cotton is replaced by BCD-BTCA-cotton). This can be expected to have an influence on wash fastness of the ester linkages crosslinking within the cotton fabric. 


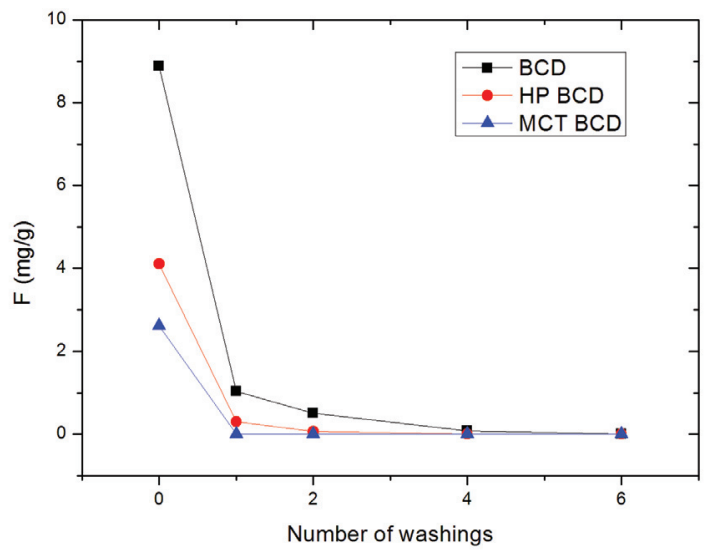

Figure 7.10: Influence of increasing number of laundering cycles on $\beta$-cyclodextrins functionalized cotton.

In addition it was seen from FTIR-ATR spectra measurements that the presence of BCD appears to influence the relative amount of ester formed in comparison to a situation with only BTCA. The resultant overall lower covalent bonds in the system also explains the poor fixation of the BCD and $\mathrm{HP} B C D$ to cotton.

It is known that ester links are susceptible to hydrolysis in alkaline conditions but BTCA esters are reported to be resistant to such hydrolysis due to the deprotonation of the unreacted acid into the anion form which provides the required electrostatic repulsion to the hydroxide ions of the detergent near the vicinity of the ester groups [40]. The wash durability of BTCA is reported in terms of the wrinkle resistant recovery angle and durable press ratings. The durability is reported to be mainly dependent on the concentration of the catalyst used. The curing temperature and time were also said to influence this durability to an extent [11]. The low amount of catalyst used in this work therefore also contributed to the poor wash durability of the BCD and HP BCD to cotton.

Despite the reducing ester formation with the increase in BCD bulk concentration, an increase in BCD fixation was observed in the fixation experiments. This would therefore mean that a significant proportion of the BCD fixed at higher bulk concentration are weakly fixed through non-covalent bonds. 
The reason for low wash durability of MCT BCD was not clear since the monochlorotriazinyl groups are expected to be covalently bound to cotton. The experimental conditions given in the MCT BCD fixation recipe prescribed by Wacker chemie was concluded to be not optimal. MCT BCD molecule fixation would require higher fixation temperatures than what has been currently been used in this work since monochlorotriazinyl reactive dyes require longer treatment time and higher treatment temperatures for effective fixation.

\subsection{Conclusion}

Cotton was exhaust treated with $\beta$-cyclodextrin (BCD) and two $\beta$-cyclodextrin derivatives (MCT BCD \& HP BCD). Attachment of BCD and HP BCD was done with BTCA while MCT BCD was fixed without a crosslinker. The quantification of the amount fixed was done by gravimetric method and the phenolphthalein method. The gravimetric method was found to be prone to significant error. Therefore the phenolphthalein method was chosen as the appropriate assessment method. Using this assessment method, the amount of fixation of BCD on cotton was found to be higher compared to the two derivatives. It was concluded that this was due to the substitution of the hydroxyl groups in the BCD derivatives which meant lower number of hydroxyl groups were available for the esterification reaction between these derivatives and the BTCA. In case of BCD fixation, the use of different amounts of crosslinker (BTCA) did not appear to have a significant influence on the amount of fixation on cotton.

FTIR-ATR spectroscopy measurements were done on BCD treated cotton. These measurements showed that the total amount of ester formed on fabric was reduced in presence of BCD in comparison to only BTCA treated cotton. The ester intensity was also seen to reduce when the BCD bulk concentration increased during the treatment. The same trend was observed when curing conditions were changed. From these measurements it was concluded that the presence of BCD hindered the esterification reaction.

DSC measurements showed no significant differences in the thermal decomposition peak temperatures due to the treatment of cotton with BTCA or BTCA-BCD as 
compared to blank cotton. This was considered to be due to the low amount of phosphorous catalyst used during the fixation.

The laundering tests showed that the $\beta$-cyclodextrins attached to cotton fabric were not wash durable. This was attributed to the lower amount of ester formation and consequently fewer ester linkages within the fabric in presence of BCD \& HP BCD as compared to the only BTCA fixation.

\section{References}

1. Roquette, Technical sheet Kleptose Betacyclodextrins and hydroxypropyl betacyclodextrins.

2. $\quad$ http://www.cyclodextrin.org/cdpdf/cs_w7_mct.pdf. last accessed date: 1-92014.

3. Reuscher, H. and R. Hirsenkorn. BETA W7 MCT - new ways in surface modification. in Proceedings of the Eighth International Symposium on Cyclodextrins. 1996. Springer.

4. Yang, C.Q. and X. Wang, Infrared spectroscopy studies of the cyclic anhydride as the intermediate for the ester crosslinking of cotton cellulose by polycarboxylic acids. III. Molecular weight of a crosslinking agent. Journal of Polymer Science Part A: Polymer Chemistry, 1997. 35(3): p. 557-564.

5. Yang, C.Q., FT-IR spectroscopy study of the ester crosslinking mechanism of cotton cellulose. Textile Research Journal, 1991. 61(8): p. 433-440.

6. Lu, Y. and C.Q. Yang, Fabric yellowing caused by citric acid as a crosslinking agent for cotton. Textile research journal, 1999. 69(9): p. 685-690.

7. Blanchard, E.J., E.E. Graves, and P.A. Salame, Flame resistant cotton/polyester carpet materials. Journal of Fire Sciences, 2000. 18(2): p. 151-164.

8. Wu, X., C.Q. Yang, and Q. He, Flame retardant finishing of cotton fleece: part VII. Polycarboxylic acids with different numbers of functional group. Cellulose, 2010. 17(4): p. 859-870. 
9. Medronho, B., et al., Cyclodextrin-Grafted Cellulose: Physico-Chemical Characterization. Carbohydrate polymers, 2012. 93(1): p. 324-330.

10. Xiaohong, G. and C.Q. Yang, FTIR spectroscopy study of the formation of cyclic anhydride intermediates of polycarboxylic acids catalyzed by sodium hypophosphite. Textile Research Journal, 2000. 70(1): p. 64-70.

11. Welch, C., Durable press finishing without formaldehyde. Textile chemist and colorist, 1990. 22(5): p. 13-16.

12. Trask-Morrell, B. and B.A.K. Andrews, Thermoanalytical ranking of catalysts for use with polycarboxylic acids as durable press reactants. Textile Research Journal, 1992. 62(3): p. 144-150.

13. Gillingham, E.L., D.M. Lewis, and B. Voncina, An FTIR study of anhydride formation on heating butane tetracarboxylic acid in the presence of various catalysts. Textile Research Journal, 1999. 69(12): p. 949-955.

14. Wei, W. and C.Q. Yang, Predicting the performance of durable press finished cotton fabric with infrared spectroscopy. Textile Research Journal, 1999. 69(2): p. 145-151.

15. Jocić, D., A. Tourrette, and P. Krizman Lavrić, eds. Biopolymer-Based StimuliResponsive Polymeric Systems for Functional Finishing of Textiles. in "Biopolymers" (M.M. Elnashar Ed.). 2010, SCIYO, Rijeka, Croatia, . 37-40

16. Šauperl, O. and K. Stana-Kleinschek, Differences between cotton and viscose fibers crosslinked with BTCA. Textile Research Journal, 2010. 80(4): p. 383-392.

17. Lee, E.S. and H.J. Kim, Durable press finish of cotton/polyester fabrics with 1, 2, 3, 4-butanetetracarboxylic acid and sodium propionate. Journal of applied polymer science, 2001. 81(3): p. 654-661.

18. Welch, C.M. and B.K. Andrews, Catalysts and processes for formaldehyde-free durable press finishing of cotton textiles with polycarboxylic acids. 1990, Patent number 4936865 . 
19. Moldenhauer, J.P. and H. Reuscher. Textile finishing with MCT-betacyclodextrin. in 9th Int. Symp. Cyclodextrins. 1999.

20. Basappa, C., P. Rao, and D.N. Rao, A modified colorimetric method for the estimation of $\beta$-cyclodextrin using phenolphthalein. International journal of food science \& technology, 1998. 33(6): p. 517-520.

21. Agrawal, P. and M. Warmoeskerken, Permanent fixation of $\beta$-cyclodextrin on cotton surface - An assessment between innovative and established approaches. Journal of applied polymer science, 2012.

22. http://www.xula.edu/chemistry/crsorgleclab/Solomons_Organic_Drill_Manual_2007/Solomons_Organic_1_fifth_ed_20 07_02nd_module_IR_table.pdf. last accessed date: 23-12-14.

23. Morris, N.M., E.A. Catalano, and B.A.K. Andrews, FT-IR Determination of degree of esterification in polycarboxylic acid cross-link finishing of cotton. Cellulose, 1995. 2(1): p. 31-39.

24. Yang, C.Q., Characterizing ester crosslinkages in cotton cellulose with FT-IR photoacoustic spectroscopy 1. Textile Research Journal, 1991. 61(5): p. 298-305.

25. Yang, C.Q. and B. Andrews, Infrared spectroscopic studies of the nonformaldehyde durable press finishing of cotton fabrics by use of polycarboxylic acids. Journal of applied polymer science, 1991. 43(9): p. 1609-1616.

26. Morris, N., B.A.K. Andrews, and E. Catalano, Determination of polycarboxylic acids on cotton fabric by FT-IR spectroscopy. Textile chemist and colorist, 1994. 26(2): p. 19-21.

27. Chung, C., M. Lee, and E.K. Choe, Characterization of cotton fabric scouring by FT-IR ATR spectroscopy. Carbohydrate polymers, 2004. 58(4): p. 417-420.

28. Sauperl, O., et al., Application of spectrophotometric methods in assessing the influence of alkaline treatment on the degree of crosslinking of cotton cellulose with BTCA. Croat. Chem. Acta, 2003. 76(4): p. 293-298. 
29. Yang, C.Q. and D. Wang, Evaluating ester crosslinking of cotton fabric by a polycarboxylic acid using acid-base titration. Textile Research Journal, 2000. 70(7): p. 615620.

30. $\mathrm{Xu}, \mathrm{W}$. and $\mathrm{Y}$. Li, Crosslinking analysis of polycarboxylic acid durable press finishing of cotton fabrics and strength retention improvement. Textile Research Journal, 2000. 70(7): p. 588-592.

31. Blanchemain, N., et al., Methyl- $\beta$-cyclodextrin modified vascular prosthesis: Influence of the modification level on the drug delivery properties in different media. Acta Biomaterialia, 2011. 7(1): p. 304-314.

32. Si, H., et al., Preparation of cyclodextrin grafting wood flour and investigation of the release characteristics of eugenol. Wood Science and Technology, 2013. 47(3): p. 601-613.

33. Hoydonckx, H.E., et al., Esterification and transesterification of renewable chemicals. Topics in Catalysis, 2004. 27(1-4): p. 83-96.

34. Wang, T., B. Li, and H. Si, Preparation of regenerate cellulose bead and its coating with cyclodextrins Cellulose chemistry and technology, 2013. 47(1-2): p. 37-47.

35. Wu, X., Flame retardant finishing of cotton fleece. PhD dissertation, University of Georgia, 2008.

36. Lecoeur, E., et al., Flame retardant formulations for cotton. Polymer degradation and stability, 2001. 74(3): p. 487-492.

37. Soares, S., G. Camino, and S. Levchik, Comparative study of the thermal decomposition of pure cellulose and pulp paper. Polymer degradation and stability, 1995. 49(2): p. 275-283.

38. Zhu, P., et al., A study of pyrolysis and pyrolysis products of flame-retardant cotton fabrics by DSC, TGA, and PY-GC-MS. Journal of analytical and applied pyrolysis, 2004. 71(2): p. 645-655.

39. Hirata, T. and T. Nishimoto, DSC, DTA, and TG of cellulose untreated and treated with flame-retardants. Thermochimica acta, 1991. 193: p. 99-106. 
40. Shekarriz, S., et al., Surface chemical analysis of 1, 2, 3, 4-butanetetracarboxylic acid modified cotton. Journal of materials science, 2003. 38(13): p. 2945-2951. 
Chapter 8 Antimicrobial functionalization of BCD

treated fabrics 


\subsection{Introduction}

In chapter 7 it was concluded that among the three types of $\beta$-cyclodextrins selected the unmodified $\beta$-cyclodextrin (BCD) was the best type to be attached to cotton. The attachment of BCD on to cotton was done via a crosslinker; 1, 2, 3, 4 butane tetracarboxylic acid (BTCA). This fixation was achieved via the formation of ester bonds between the hydroxyl groups of $\mathrm{BCD}$ and cotton with the acid groups of the crosslinker though the esterification reaction. It is not possible to attach $\mathrm{BCD}$ to a polyester surface by this reaction due to the lack of reactive groups on the surface of polyester. Reactive groups can be formed on the surface of polyester through a process referred to as activation. This activation can be done via chemical or physical initiation (such as microwaves, UV radiation or plasma treatment) methods to create free radicals on the backbone of the polymeric substrate. The surface modification is then achieved by the graft polymerization of a functional group containing monomer on to the surface of polyester. In this work, the activation was done by the UV grafting of acrylic acid on to the polyester substrate. The carboxylic groups of the grafted acrylic acid on polyester were expected to react with the hydroxyl groups of the $\mathrm{BCD}$ molecules through hydrogen bonding during the subsequent $\mathrm{BCD}$ fixation experiments [1]. Additionally an esterification reaction was expected between the carboxylic (of activated polyester) and hydroxyl groups (of BCD) under curing conditions. The literature reports that polyacrylic acid as a crosslinker (in presence of a catalyst) is not as effective as BTCA in esterification reactions with cotton [2]. In our work acrylic acid and not polyacrylic acid (since it is already a polymer and not a monomer required for the polymerization reaction in the activation step) the was used for the grafting however the esterification reaction is expected to be identical in its mechanism. Therefore the attachment of BCD to activated polyester was not expected to result in a high amount of BCD fixation. The reason for the lower effectiveness of polyacrylic acid as a crosslinker is due to its inability to form a five member anhydride intermediate as in the case of BTCA (explained in chapter 7). Therefore in this work, the crosslinker BTCA was additionally added to the treatment bath to increase the amount of BCD fixed to the activated polyester. The addition of BTCA into the liquor increases the solubility of BCD (which is $18.6 \mathrm{~g} / \mathrm{l}$ ) in the liquor due to which the BCD fixation on to APES can be expected to increase. The 
catalyst sodium hypophosphite (SHPI) was also added into the liquor and its role in the esterification reaction was already explained in chapter 7 .

After the fixation of $\mathrm{BCD}$ to the various substrates, the antibacterial functionalization of the substrates was done. As explained in section 6.7 of chapter 6 , the antibacterial molecule PHMB could not be used in the multi-step method due to its high water solubility and large molecular weight. As described in section 6.2 of chapter 6, an ideal guest molecule should have a molecular weight between 100-400 g/mol and water solubility of less than $10 \mathrm{~g} / \mathrm{l}$ in order to form a complex with the BCD molecule. A new guest molecule with a relatively lower molar mass with suitable geometry and hydrophobicity was required in order to have a complex with the BCD cavity. The disinfectant chlorhexidine was selected based on these requirements. Section 8.2.3 of this chapter describes this choice in detail. Chlorhexidine is available as a base and in its various salt forms. Some of the commercially available chlorhexidine salts are chlorhexidine dihydrochloride, chlorhexidine diacetate and chlorhexidine digluconate. Chlorhexidine diactetate was chosen as the antibacterial agent for the multi-step work. The reasons for this choice is further explained in section 8.6. In this work, the term 'chlorhexidine' refers to the molecule in general, i.e. its base and salt forms. While the acronym $\mathrm{CHXB}$ is used while referring to the chlorhexidine base molecule and CHXA refers to the diacetate salt of chlorhexidine.

\subsection{Materials}

The plain woven bleached cotton, polyester (PES) and blends of fabric density 170 $\mathrm{g} / \mathrm{m}^{2}$ was obtained from WFK Testgewebe, Germany. The blends were a mix of cotton and polyester with a composition ratio of $35 \%$ to $65 \%$ respectively. The BCD was obtained from Roquette, France. BTCA, sodium hydroxide, sodium carbonate, Tris(hydroxymethyl) aminomethane (Tris), phenolphthalein, sodium hypophosphite (SHPI), chlorhexidine base (CHXB) and chlorhexidine diacetate (CHXA) were obtained from Sigma Aldrich.

\subsection{Activation of polyester}

As mentioned earlier the activation of PES can be done by the UV grafting of acrylic acid. PES samples measuring $50 \mathrm{~cm}$ by $50 \mathrm{~cm}$ were treated in large glass oven dish tray containing a bulk solution of $70 \% \mathrm{v} / \mathrm{v}$ of acrylic acid, $30 \%$ water and $5 \% \mathrm{w} / \mathrm{v}$ of 
the photoinitiator benzophenone. An LCR of 10:1 was used. The bulk liquor in which textile sample were treated was continuously stirred with magnetic stirrers. The activation was done at room temperature for a duration of 5 minutes. The sample was then taken out of bulk solution and placed under a UV lamp for 2 minutes. The sample was kept at a distance of $10 \mathrm{~cm}$ from the lamp. Both sides of the sample were radiated under the UV lamp. The sample was then washed for 60 minutes in $40^{\circ} \mathrm{C}$ demi water and rinsed to remove the unreacted acrylic acid and the acrylic acid homopolymer (side product formed during the activation). The sample was then air dried. Figure 8.1 shows the grafting of the carboxylic groups of the acrylic acid on to the surface of PES after the activation step [3]. Such an activated polyester is henceforth referred to in this work as APES.<smiles>C=CC(=O)O</smiles>

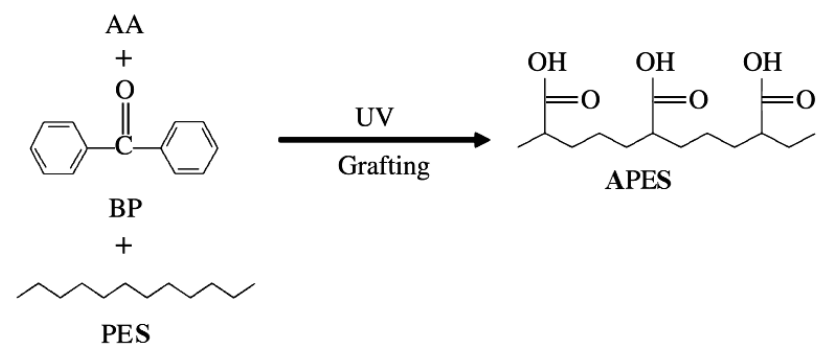

Figure 8.1: Activation of polyester with UV grafting of acrylic acid (here AA refers to acrylic acid, BP refers to benzophenone, PES refers to polyester,. APES refers to activated polyester) and UV irradiation. (Reproduced from Song. Y.W et al. [3]with permission from [Taylor \& Francis]).

\subsubsection{Surface chemical analysis of activated polyester with XPS}

The XPS analysis was done with Quantera SXM (scanning XPS microprobe) from Physical Electronics to investigate the surface chemical changes on the activated sample as compared to a control sample. The samples were irradiated with monochromatic $\mathrm{Al} \mathrm{Ka}$ X-rays $(1486.6 \mathrm{eV})$ using a beam with diameter of $200 \mu \mathrm{m}$ and a power of $50 \mathrm{~W}$. 


\subsection{Fixation of BCD to the various textile substrates}

PES, APES and blends were treated with varying concentrations of BCD and BTCA. All fabrics were treated with BCD and BTCA via the exhaust method with the procedure described in section 7.2.2.1 of chapter 7. All the fixation treatments were done in triplicate.

As explained earlier BTCA was also added into the liquor bath during the treatment of APES with BCD. The addition of BTCA increased the solubility of BCD from 18.6 $\mathrm{g} / 1$ to more than $100 \mathrm{~g} / 1$. This was expected to increase the fixation amount of BCD on the surface of the APES samples.

The activation of PES and the fixation of BCD to APES was not combined into a onebath method where all reactants were added into the bath at the same time. This was because a better efficiency (in terms of grafting of acrylic acid and the amount of BCD fixed) was expected in the separated activation and subsequent BCD fixation method as compared to a one-bath method. It was considered that in the one-bath method the water soluble acrylic acid would show more affinity towards the polar hydroxyl groups of BCD than to the hydrophobic PES substrate. In earlier work with the attachment of hydrogels to activated polyester films, it was observed that a separate activation and fixation method showed a higher hydrogel fixation as compared to the one-bath method.

\subsubsection{Quantification of fixed BCD on the various substrates}

Quantification of the BCD fixed on to the various substrates was done with the phenolphthalein method as described in section 7.3.2 of chapter 7. As mentioned in chapter 7 , the determination of BCD fixation amount from the phenolphthalein method assumes that all of BCD cavities fixed on the fabric are accessible to the phenolphthalein molecules. Any steric influence contributed by the crosslinker on the BCD-phenolphthalein absorbance is not considered since the calibration curve used for the calculations are of only the BCD-phenolphthalein solutions. Additionally the instability in the BCD-phenolphthalein absorbance due to the unreacted acid groups on the fabrics is also ignored as already discussed in chapter 7. 


\subsubsection{Moisture content measurements of BCD treated samples}

Moisture content measurements were done to access the influence of the $\mathrm{BCD}$ fixation on the moisture content of the various fabrics. Moisture content measurements were done on MS-70 moisture analyzer equipped with WinCTMoisture software. All the samples were conditioned for 24 hours at $20^{\circ} \mathrm{C}$ and $65 \%$ relative humidity. Then the samples were placed on heating plate of the analyzer and heated to $105^{\circ} \mathrm{C}$. The weight of the sample was measured before and after the heating. From the differences in this weight, the moisture content \% was calculated as shown in Equation 8.1. These moisture content measurements were done for cotton, blends, PES and APES samples treated with $30 \mathrm{~g} / 1$ of BTCA (samples fixed with BCD with $30 \mathrm{~g} / 1$ of BTCA showed better repeatability than the other two BTCA concentrations).

Moisture content $\%=\frac{(W-D)}{W} .100 \%$

Where $W$ is the weight of sample at the start of the experiment and $D$ is the weight of sample at the end of experiment.

\subsection{UV vis spectrophotometer measurements of $C H X B-B C D$ and CHXA-BCD solutions}

\subsubsection{Introduction to chlorhexidine}

As mentioned earlier, chlorhexidine was chosen as the guest antibacterial agent for the complexation with the BCD molecules in the multi-step work. The choice of this agent was made on the basis of its hydrophobicity, molar mass and its well-known ability to complex with the BCD cavity [4]. Chlorhexidines are chemically bisguanides and are therefore closely related to polymeric biguanides such as PHMB. Similar to PHMB, the cationic chlorhexidines are attached to a surface of the anionic textiles through electrostatic interaction [5, 6]. Again as with PHMBs, chlorhexidines are membrane attacking agents and the risk of the development of microbe resistance is reported to be much lower than the more widely used quaternary ammonium compounds [7]. 
Chlorhexidine is commercially synthesized in its base and salt forms. The structure of chlorhexidine in its base form (CHXB) is given in Figure 8.2a. The molar mass of this base molecule is $505.45 \mathrm{~g} / \mathrm{mol}$.<smiles>[H][Y8]([H])(C(=N)NC(=N)NCCCCCCNC(=N)NC(=N)Nc1ccc(Cl)cc1)c1ccc(Cl)cc1</smiles>

Figure 8.2a: Structure of chlorhexidine base (CHXB).

Chlorhexidines have a molecular dimension of $28.55 \AA$ while the length of a BCD cavity is $7.8 \AA$. The entire length of a chlorhexidine molecule cannot fit into the BCD cavity. However, the radial dimensions of the phenyl rings of the chlorhexidine molecule are small enough to fit width-wise inside the BCD cavity $[8,9]$. Therefore only the two hydrophobic end phenyl rings of the chlorhexidine molecule complex inside the BCD cavities which results in a complexation ratio of 1:2 (between the chlorhexidine and the BCD molecules). This is shown in Figure 8.2b. It is also reported that this model may however be too simplistic and in reality one or more BCD molecules can additionally thread through the chlorhexidine molecule leading to a complexation ratio of more than 1:2 [4].

The most commonly used chlorhexidine type in disinfectant solutions are the salt forms such as chlorhexidine diacetate (CHXA), chlorhexidine digluconate (CHXG) and chlorhexidine dihydrochloride $(\mathrm{CHXH})$. This is due to the improved stability and solubility of the salt forms as compared to the base molecule. Among the various chlorhexidine salt forms available, the salt CHXA was considered to be interesting for this work. The water solubility of this salt (as compared to the base and the other salts) was particularly suitable for the textile exhaust applications. 


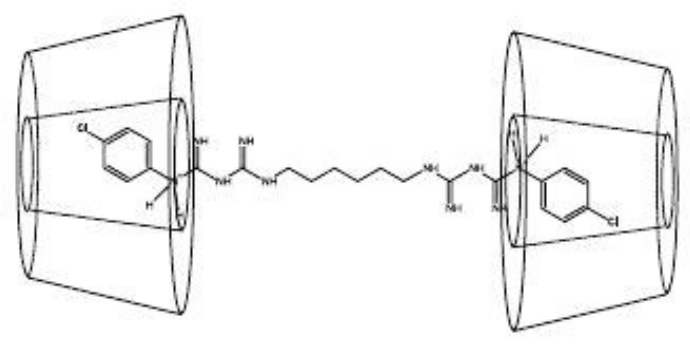

Figure 8.2b: Complexation of chlorhexidine base (CHXB) with BCD molecules. (Reproduced from Denadai et al. [8] with permission from [Elsevier]).

This is explained further in section 8.6. CHXA has a molecular weight of $625.6 \mathrm{~g} / \mathrm{mol}$ [10]. The structure of CHXA is given in Figure 8.2c.

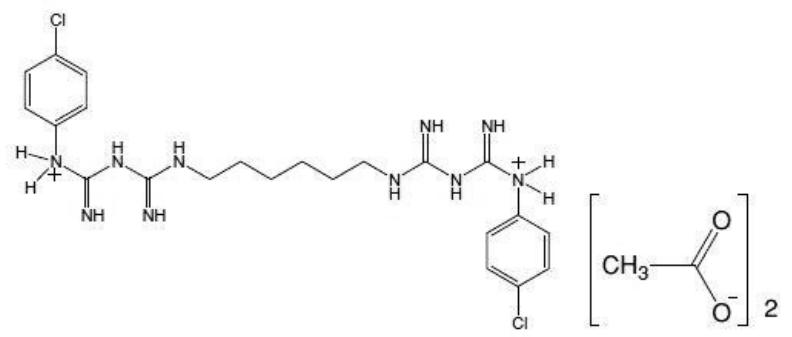

Figure 8.2c: Structure of CHXA.

\subsubsection{UV vis spectrophotometric studies of CHXB-BCD and CHXA-BCD solutions}

UV vis spectrophotometer studies are done with BCD-guest solutions to study the stability or binding of the complexation [4]. The basic principle in such spectral studies is to measure the change in the UV vis spectrophotometric maximum absorbance of the aqueous guest molecule before, during and at the end of complexation with the BCD molecules. During the measurements, the concentration of the guest molecules in the solution is kept constant while that of the BCD is steadily increased. As the guest molecules begin to complex with the BCD molecules, a shift is seen in the maximum absorbance peak. This is due to the complexation of the chromophore moieties of the guest molecule inside the BCD cavity. The absorbance value of this shifted peak increases with the concentration of the BCD in the solution. This continues until no further complexation takes place upon the 
addition of (excess) BCD into the solution.

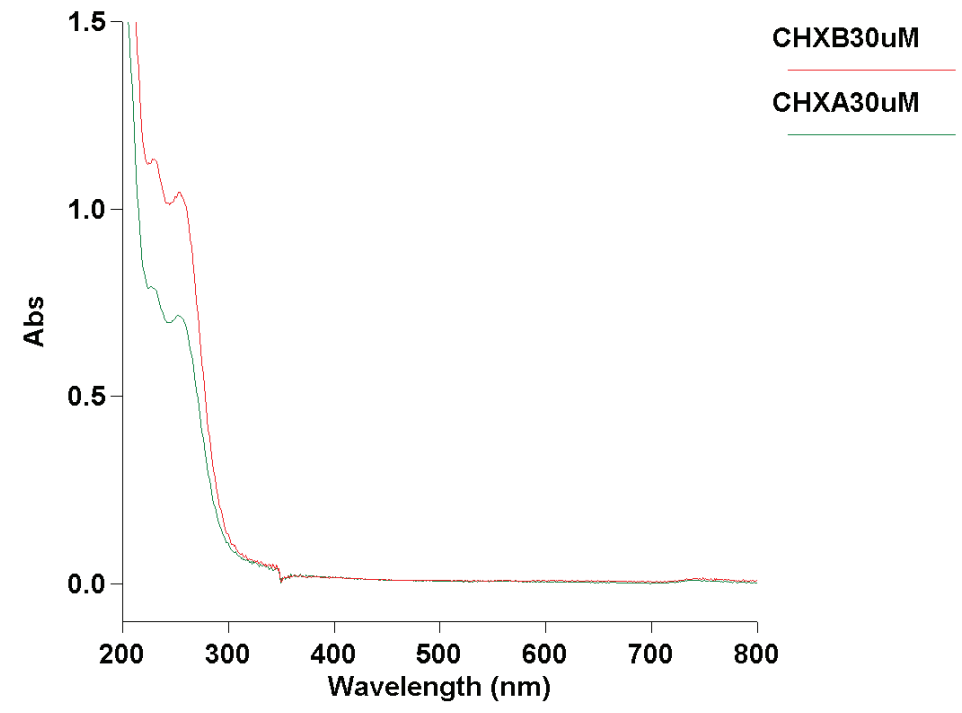

Figure 8.3: The UV vis spectrophotometric absorbance scans through 200-800nm of aqueous solutions of $\mathrm{CHXB}$ and $\mathrm{CHXA}$. The concentration of CHXA and CHXB is 30 $\mu \mathrm{M}$.

We hypothesized that due to the differences in water solubility and molar mass between CHXA and CHXB molecules, the trend in the complexation between the two types of chlorhexidine molecules and the BCD molecules would not be the same. This would be easily observable in the UV vis spectral studies where the complexation would result in a change in the maximum absorbance.

The UV vis spectrophotometer scans of CHXB and CHXA in aqueous solutions showed two absorbance peaks; at $234 \mathrm{~nm}$ and at $254 \mathrm{~nm}$ as seen in Figure 8.3. In this work the absorbance peak at $254 \mathrm{~nm}$ was used for all the experiments as it appeared to be sharper than the peak at $234 \mathrm{~nm}$.

Stock solutions of CHXA $(60 \mu \mathrm{M}), \mathrm{CHXB}(60 \mu \mathrm{M})$ and BCD $(15 \mathrm{mM}$ which is the solubility limit of BCD in water) were made with demi water in $100 \mathrm{ml}$ beakers. A series of test tube containing a mix of the aqueous solutions of $\mathrm{CHXB}$ with $\mathrm{BCD}$ solutions were then prepared. The final concentration of the CHXB solution in the test tubes was kept constant at $30 \mu \mathrm{M}$ while the concentration of the BCD solution 
was varied from 0 to $7.5 \mathrm{mM}$. These solutions were then measured for their UV vis spectra with the UV vis spectrophotometer (Cary 100, USA). These measurements were conducted at room temperature and at neutral $\mathrm{pH}$. This was then followed by the UV vis measurements for CHXA in BCD solutions in the same manner.

\subsection{Treatment of cotton, BCD-cotton, APES and BCD-APES fabrics with CHXA}

Cotton and APES substrates treated with $100 \mathrm{~g} / 1$ of BCD and $30 \mathrm{~g} / 1$ BTCA were chosen for the antibacterial functionalization (referred to henceforth as BCD-cotton and APES-cotton). This was due to the reason that fabrics treated with a BCD bulk concentration of $100 \mathrm{~g} / 1$ showed the highest amount of BCD fixed on the various textiles as compared to the other bulk concentrations in this work. The amount of BCD fixed on cotton for the antibacterial functionalization work was calculated from the phenolphthalein method prior to the experiments. The amount of BCD fixed was $6.2 \mathrm{mg}$ per gram and $8.3 \mathrm{mg}$ per gram of cotton and APES fabrics respectively.

CHXA was chosen as the guest antibacterial agent for the antibacterial treatment due to its more suitable aqueous solubility and molecular weight as compared to the other salts. The solubility of CHXA is $19 \mathrm{~g} / 1(1.9 \% \mathrm{w} / \mathrm{v})$ in comparison to $0.08 \mathrm{~g} / 1$ $(0.008 \% \mathrm{w} / \mathrm{v})$ of CHXB [11]. This higher water solubility would allow a wide range of application bulk concentrations while using water as a solvent. In case of $\mathrm{CHXB}$ a solvent such as ethanol would have to be considered for higher application bulk concentrations. As explained in chapter 1 and chapter 6, the multi-step work when feasible, is meant to be applied in an industrial laundry. In the industrial laundry the application of the guest molecule is to be realized in the rinsing step of the laundering cycle where the solvent is water. On the other hand, the CHXA molecule is not as hydrophobic as CHXB. The other two salts of chlorhexidines, CHXG and $\mathrm{CHXH}$ were not chosen due to their inability to match the solubility requirements. The aqueous solubility of CHXG is more than $700 \mathrm{~g} / 1(>70 \% \mathrm{w} / \mathrm{v})$ [11] and its molecular weight is $897.8 \mathrm{~g} / \mathrm{mol}$. The molecular weight and water solubility parameters appear to lie wide outside the limits set for guest molecule requirements for effective complexation with the BCD molecules. These limits were described earlier in section 6.2 of chapter $6 . \mathrm{CHXH}$ on the other hand has a lower molecular weight at $578.37 \mathrm{~g} / \mathrm{mol}$. The aqueous solubility is also low at $0.6 \mathrm{~g} / 1(0.06 \% \mathrm{w} / \mathrm{v})$ 
[11]. Though this is acceptable since the upper limit of the solubility of a required guest molecule is $10 \mathrm{~g} / 1$ (for complexation formation with BCD), this would however imply that a wide (and high) range of bulk concentrations treatments would not possible while using water as a solvent. Exhaust treatments with a wide range of bulk concentrations would allow the treatment of fabrics with a wide range of antibacterial activity. As a tradeoff between the hydrophobicity of the molecule and its aqueous solubility, the latter was given a priority. Therefore as a starting point, CHXA was chosen as the guest molecule among the various chlorhexidine salts for the multi-step work.

The treatment of cotton, BCD-cotton, APES and BCD-APES with CHXA was done in a beaker with an LCR of 10:1. The dimensions of the textile samples were of $10 \mathrm{~cm}$ by $10 \mathrm{~cm}$. The experimental conditions were the same as earlier with the treatment of the textiles with PHMB (in chapter 4). The treatment of the fabrics was done at a temperature was $40^{\circ} \mathrm{C}$ and at neutral $\mathrm{pH}$. The duration of treatment was kept at 30 minutes. The application bulk concentrations of CHXA ranged from $0.01 \mathrm{~g} / 1$ to 2.5 $\mathrm{g} / 1$.

After the treatment of the various samples in the CHXA bulk liquor, the fabrics were gently removed from bulk liquor. The UV vis absorbance of the bulk was then measured. The concentration of CHXA in the bulk was finally determined by using the calibration curve shown in Figure 8.4.

It is seen in Figure 8.4 that a linear curve can be obtained between 1 ppm to $25 \mathrm{ppm}$ $(0.001 \mathrm{~g} / 1$ to $0.025 \mathrm{~g} / 1)$ of CHXA. However the treatment of the fabrics with CHXA ranged from bulk concentrations of $0.001 \mathrm{~g} / 1$ to $2.5 \mathrm{~g} / 1$. The CHXA concentrations in the bulk liquor after the treatment were much higher than the measurable limit shown in the calibration curve.

The exhaust bulk solutions after the textile treatment were diluted by 10-1000 times and the UV vis absorbance were then measured. Then the concentrations in the beaker were recalculated using the calibration curve after taking into consideration the dilution factor. 
From the difference between the known bulk concentration at the start of the experiment and the calculated bulk concentrations at the end of the experiment, the adsorption on to the textile could be calculated as shown in Eauation 8.2.

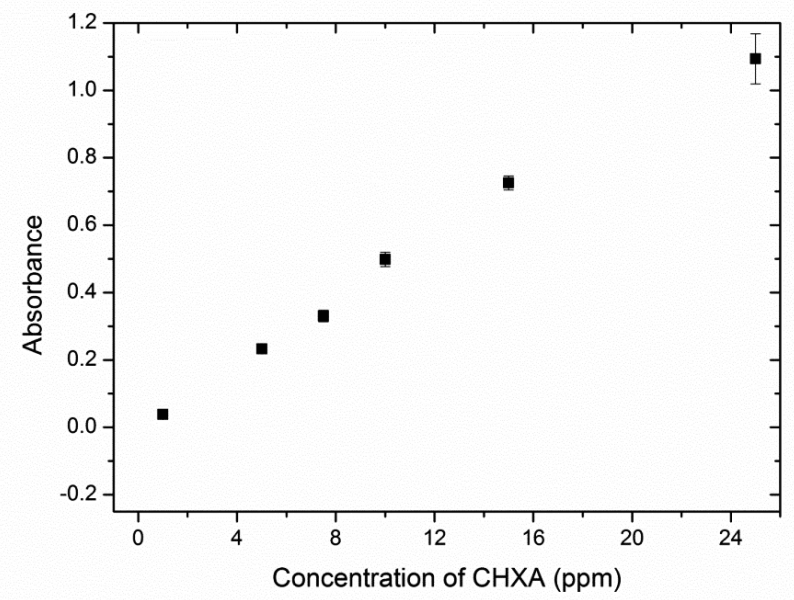

Figure 8.4: Calibration curve of CHXA. Here A refers to the absorbance. The absorbance value were measured with $U V$ vis spectrophotometer at $254 \mathrm{~nm} .1 \mathrm{ppm}$ $=0.001 \mathrm{~g} / 1$.

CHXA adsorbed $\left(\frac{m g}{g}\right)=\left((C i-C f) \cdot \frac{V \cdot 1000}{W}\right)$

CHXA refers to chlorhexidine diacetate, $C i$ refers to the initial bath concentration of CHXA, $C f$ refers to the final bath concentration of CHXA, $V$ refers to the liquor volume in 1 and $W$ is the mass of the fabric in $g$.

The BCD-APES fabric treated with CHXA is henceforth referred to as BCD-APESCHXA and BCD-cotton treated with CHXA is referred to as BCD-cotton-CHXA. The respective control fabrics treated with $\mathrm{CHXA}$ are the cotton-CHXA and APES-CHXA fabrics.

\subsection{Antibacterial tests of CHXA treated fabrics}

Chlorhexidine is a broad spectrum antibacterial agent effective against gram negative and gram positive bacteria. As with $\mathrm{PHMB}$, it is a membrane active agent and the primary attack site is the bacteria cell membrane. The chlorhexidine molecule as mentioned earlier has a molecular dimension of $28.55 \AA$ while an E coli bacterium 
measures $0.5 \mu \mathrm{M}$ by $2 \mu \mathrm{M}$. The chlorhexidine molecule therefore is approximately a 1000 times smaller than an E coli bacterium. The cationic biguanide groups of chlorhexidine have affinity for the negatively charged bacterial cell wall. These anionic sites on the cell membrane stabilize the divalent cations on the membrane surface. Figure 8.5 shows the chlorhexidine antibacterial mechanism.

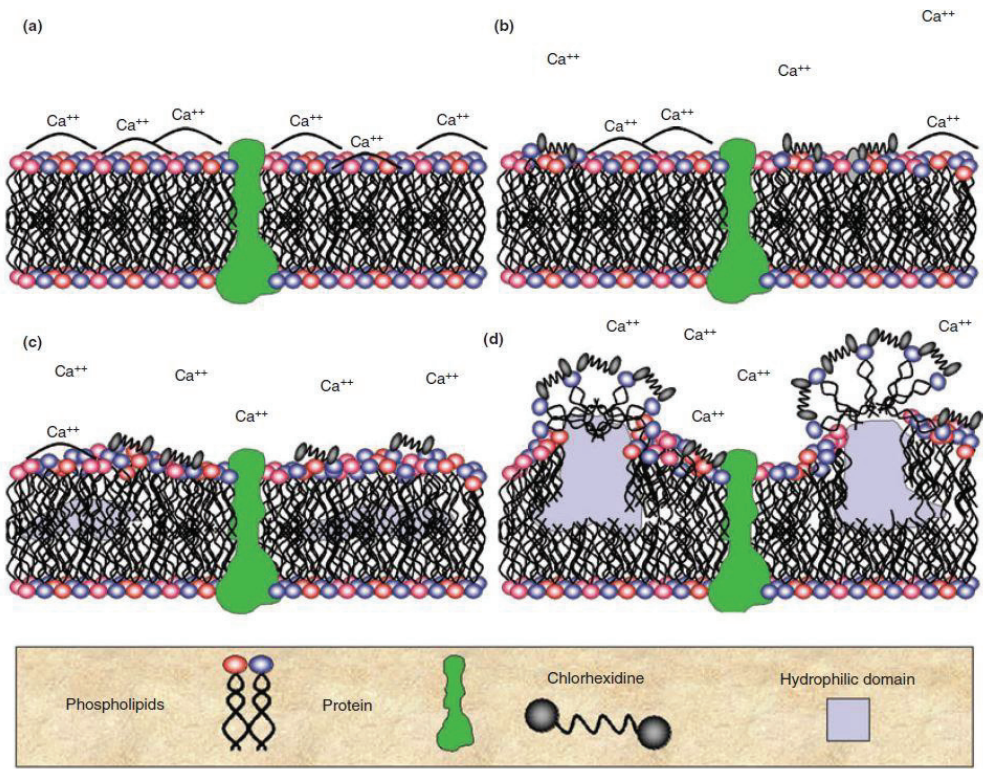

Figure 8.5: The attack of bacterial cell membrane by chlorhexidine (Reproduced from Gilbert et al. [7] with permission from [John Wiley and sons]).

The first step of the attack is the destabilization of the divalent cations on the membrane surface and the settlement of the end groups of chlorhexidine on top of two phospholipid heads of the membrane bilayer. The hexamethylene structure is relatively inflexible due to being only six carbons long and therefore does not enter the phospholipid bilayer (similar to PHMB). The antibacterial molecule then proceeds to solubilize the hydrophobic membrane leading to cellular leakage and finally the structural disintegration of the bacterium [7].

The antibacterial tests were done on BCD-cotton-CHXA and BCD-APES-CHXA samples following the JIS L 1902 standard [12]. The tests were done with E coli 11229 strain [13]. These experiments were done as described in section 3.2.4 of chapter 3. The antibacterial activity, AA was calculated according to Equation 2.2 of chapter 2. 


\subsection{The release of CHXA from BCD-cotton-CHXA and cotton-CHXA fabrics}

The results of the antibacterial tests (discussed in section 8.9.6) showed that BCDcotton-CHXA and BCD-APES-CHXA samples had lower antibacterial activity as compared to cotton-CHA and APES-CHXA samples for the same bulk concentration treatments. This was attributed to the slow release phenomenon of the BCD molecules (mentioned earlier also in section 6.6 of chapter 6).

CHXA release experiments were done in order to confirm the slow release phenomenon of the BCD fixed samples. The principle underlying these experiments was to soak the CHXA treated samples in water and then spectrophotometrically measure the release of CHXA from the samples into the water. BCD-cotton-CHXA and cotton-CHXA fabrics treated with $1.5 \mathrm{~g} / 1$ of CHXA bulk concentration was chosen for the release experiments. This was because the release of CHXA into the bulk was spectrophotometrically measurable with the BCD-cotton-CHXA samples treated only at and above this CHXA bulk concentrations. Samples treated with lower CHXA bulk concentrations (below $1.5 \mathrm{~g} / \mathrm{l}$ ) showed very little release of CHXA into the bulk water which made the spectrometric measurements inconsistent.

BCD-cotton-CHXA and cotton-CHXA samples measuring 2.5 by $2.5 \mathrm{~cm}$ were placed in a beaker containing $20 \mathrm{ml}$ of demi water. The water in the beaker was kept at room temperature and at neutral $\mathrm{pH}$. The water inside the beaker was magnetically stirred. The duration of the experiment was 60 minutes. The UV vis spectrophotometer absorbance of the bulk water was measured at the earlier mentioned CHXA maximum absorbance of $254 \mathrm{~nm} .1 \mathrm{ml}$ of the bulk liquor was pipetted out for each measurement after which it was replaced back into the bulk. The measurement of the bulk was done every minute for the first 10 minutes and then every two minutes for the next 10 minutes and then for every five minutes till the end of the 60 minutes. The release of CHXA into the bulk was calculated from the calibration curve shown in Figure 8.3. 


\subsection{Results and discussions}

\subsubsection{Quantification of BCD}

The quantification of the amount of BCD fixed on the various fabrics was done with the phenolphthalein method described in chapter 7 . The results of the phenolphthalein tests are shown in Figure 8.6a-8.6c for the three BTCA concentrations; 10,30 and $60 \mathrm{~g} / 1$. As explained in chapter 7 the results are shown as $\mathrm{BCD}$ fixation amount per gram of the fabric $(\mathrm{F} ; \mathrm{mg} / \mathrm{g})$ against the various $\mathrm{BCD}$ bulk concentrations.

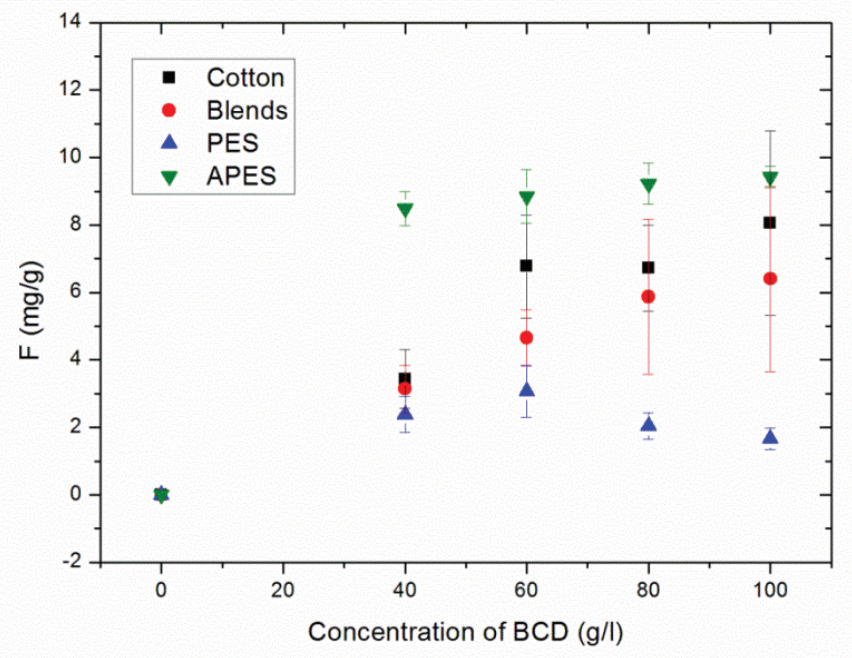

Figure 8.6a: Amount of BCD (mg/g) fixed on the different substrates treated with BCD with a BTCA concentration of $10 \mathrm{~g} / 1$. 


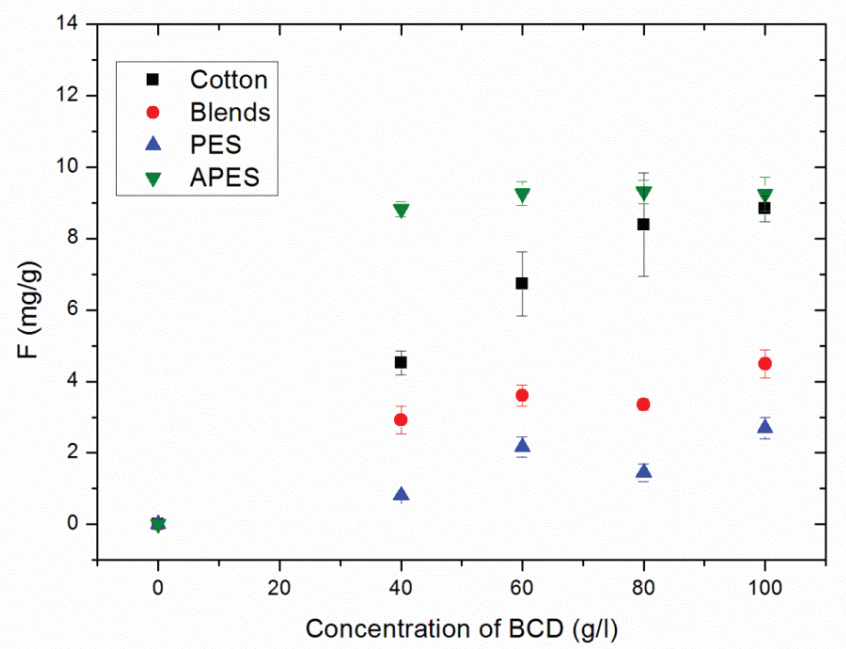

Figure 8.6b: Amount of $\mathrm{BCD}(\mathrm{mg} / \mathrm{g})$ fixed on the different substrates treated with BCD with a BTCA concentration of $30 \mathrm{~g} / 1$.

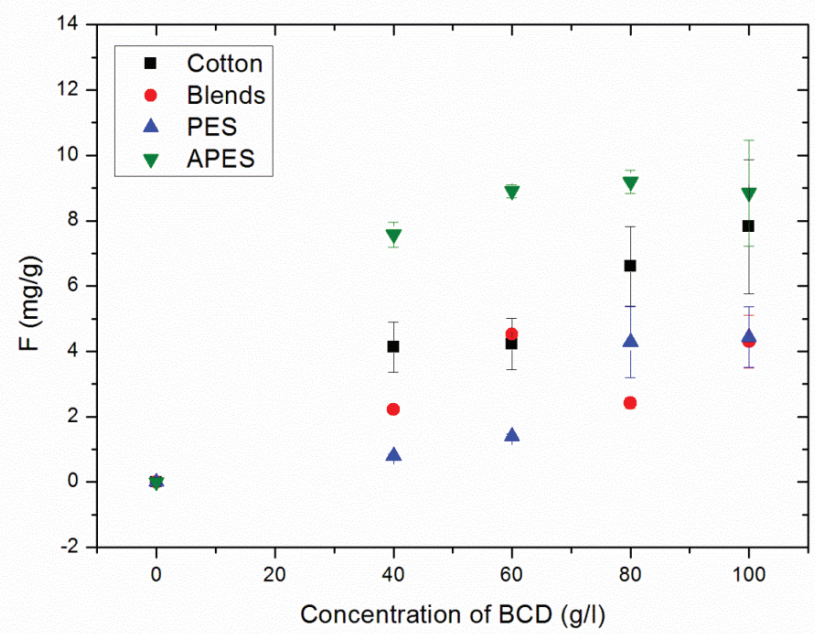

Figure 8.6c: Amount of BCD (mg/g) fixed on the different substrates treated with BCD with a BTCA concentration of $60 \mathrm{~g} / 1$.

From these figures it appears that the APES fabrics have a significantly higher amount of BCD fixed as compared to the other fabrics. This is attributed to the 
activation step which created carboxylic groups on the surface of PES and the addition of the crosslinker BTCA into the liquor. As mentioned earlier, the low aqueous solubility of BCD $(18.6 \mathrm{~g} / \mathrm{l})$ is a limiting factor in the fixation of BCD to textile surfaces where the bulk liquor is an aqueous solution. The addition of BTCA into the bulk increases the solubility of BCD to more than $100 \mathrm{~g} / 1$ and thereby improving the fixation amount. BTCA might have also contributed to the increase in fixation by proving extra carboxylic groups (for the esterification reaction) in the bulk liquor. However it is likely that these additional carboxylic groups do not contribute to the covalent fixation of BCD to APES.

As observed in chapter 7 with the BCD fixation to cotton, the amount of BTCA concentration does not have a significant influence on the fixation amount. The BCD fixation values appear to be more repeatable at $30 \mathrm{~g} / 1$ of BTCA. This concentration was considered suitable for further applications. For the antibacterial functionalization, fabrics treated with $100 \mathrm{~g} / 1$ of BCD bulk concentration was considered suitable. APES-BCD and cotton-BCD fabrics treated with this BCD bulk concentration showed a fixation of about $8 \mathrm{mg} / \mathrm{g}$ of the fabric.

From the figures it can be observed that PES shows some amount of BCD fixation with $30 \& 60 \mathrm{~g} / 1$ of BTCA despite having no reactive groups on its surface. As explained in chapter 6 , this is due to the physical entanglement of the BCD molecules within the BTCA network in the PES substrate. As expected blends show a BCD fixation amount higher than PES but lower than cotton. This is due to the reason that blends contain fewer hydroxyl groups (about $1 / 3^{\text {rd }}$ the number present on $100 \%$ cotton) than cotton for the fixation with BCD.

It was explained in section 7.6 .3 of chapter 7 that the amount of ester bonds formed on the surface of BCD-cotton reduces with the increasing BCD bulk concentration as compared to the only BTCA treated cotton. This was concluded based on the FTIRATR measurements of the ester intensities on BCD-cotton samples at the $1720 \mathrm{~cm}^{-1}$ wavenumber. These measurements were attempted on APES-BCD and APES (treated with only BTCA) samples. Relative changes in ester intensity at $1720 \mathrm{~cm}^{-1}$ wavenumber could not obtained due to the reason that the intensity at this wavenumber also covers the ester bonds of the PES substrate. These ester bonds are 
formed during the polymerization reaction between terephthalic acid and hexamethylene diamine groups to form polyester. The large peak at this wavenumber therefore made it impossible to measure repeatable quantitative differences in the FTIR-ATR ester intensities between the various treatments. It is however likely that the esterification reaction between the carboxylic groups on APES and the hydroxyl groups on BCD is negatively influenced by the steric hindrance of the large BCD molecule. As concluded in chapter 7 , it can be said that the a significant portion of the bonds involved in fixation of BCD to APES-BCD samples (especially at higher BCD bulk concentrations) would be non-covalent.

\subsubsection{Moisture content measurements}

Moisture content measurements were done on fabrics crosslinked with $30 \mathrm{~g} / \mathrm{l}$ of BTCA due to the earlier conclusion that samples treated with this cross linker concentration show repeatable results. Figure 8.7a shows the moisture content in the untreated fabrics. It can observed here that untreated APES shows a slightly higher moisture content than PES due to the presence of carboxylic groups on its surface. From Figure 8.7b, Figure 8.7c and Figure 8.7d, it can be seen that the moisture content is decreased after the attachment with only BTCA (control fabrics) with all of the substrates with the exception of PES. This reduction in moisture content is due to the conversion of the hydrophilic hydroxyl groups of untreated cotton, blends and APES into the carboxylate esters (COOR groups). PES has an inert surface and therefore no reactive groups on its surface. As mentioned earlier, the BTCA molecules can form a physical network within the PES filaments. These hydrophilic acid groups contribute to a slight gain in moisture content of the PES fabric treated with BTCA as compared to the untreated PES.

The increase in moisture content of fabrics after the treatment with BCD is due to the increase in the content of the hydroxyl groups on these fabrics which are contributed by the fixed BCD molecules. This holds valid for the PES fabrics treated with BCD as well where the BCD molecules are held within the BTCA network. 


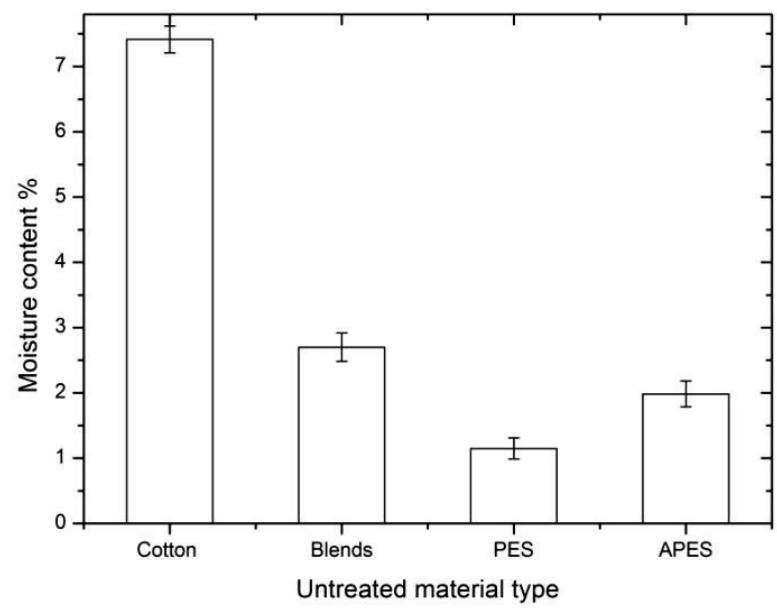

Figure 8.7a: Moisture content of the various untreated materials.

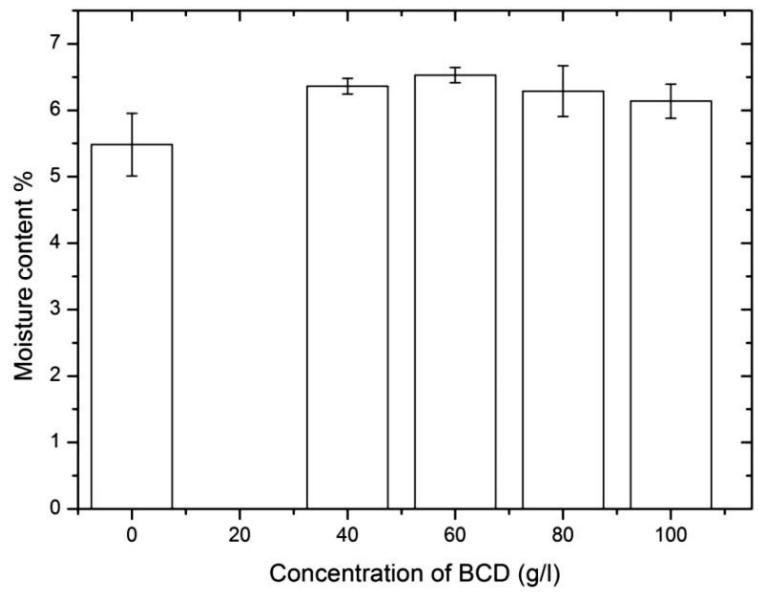

Figure 8.7b: Moisture content of cotton samples treated with BCD and $30 \mathrm{~g} / 1$ of BTCA. 


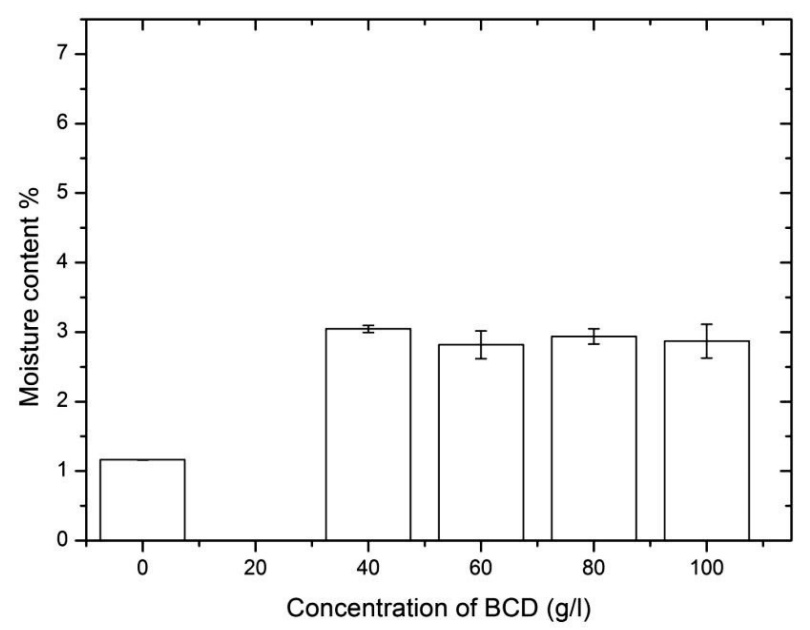

Figure 8.7c: Moisture content of polyester cotton (blends) samples treated with BCD and $30 \mathrm{~g} / 1$ of BTCA.

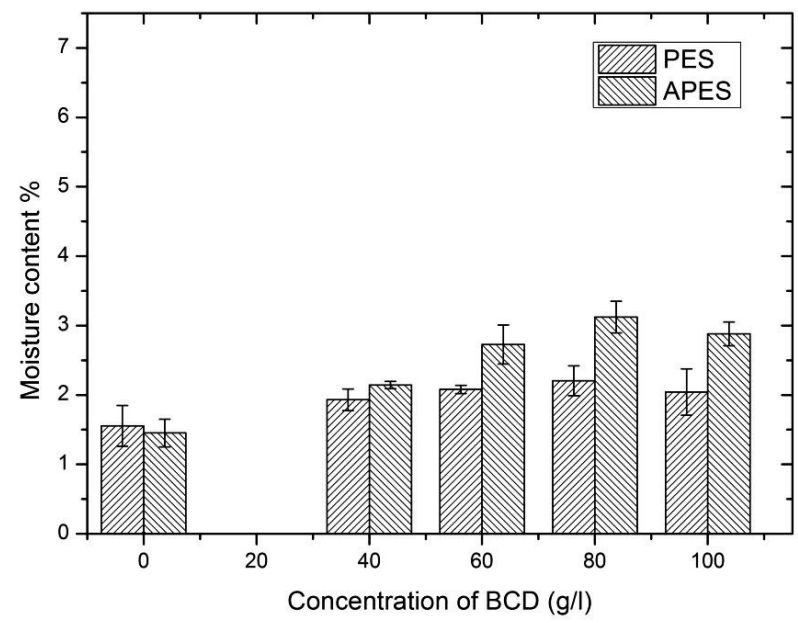

Figure 8.7d: Moisture content of PES and APES samples treated with BCD and $30 \mathrm{~g} / 1$ of BTCA.

\subsubsection{Surface chemical analysis with XPS}

XPS analysis was done on the untreated PES and the activated APES sample in order to confirm the activation of PES with AA, BP and UV radiation. The XPS spectra of PES material shows only two peaks namely the $\mathrm{C}_{1 \mathrm{~s}}$ at $285 \mathrm{eV}$ and $\mathrm{O}_{1 \mathrm{~s}}$ at $533 \mathrm{eV}$ as 
shown in Figure 8.8a. The spectral scans can be used to calculate the oxygen to carbon ratio in the material. Further the relative percentage of the type of carbon ( $\mathrm{O}=\mathrm{C}-\mathrm{O}, \mathrm{C}-\mathrm{C}-\mathrm{O}$ and $\mathrm{C}-\mathrm{C}-\mathrm{C})$ and oxygen ( $\mathrm{C}=\mathrm{O}$ and $\mathrm{C}-\mathrm{O}-\mathrm{C})$ bonds can also be determined.

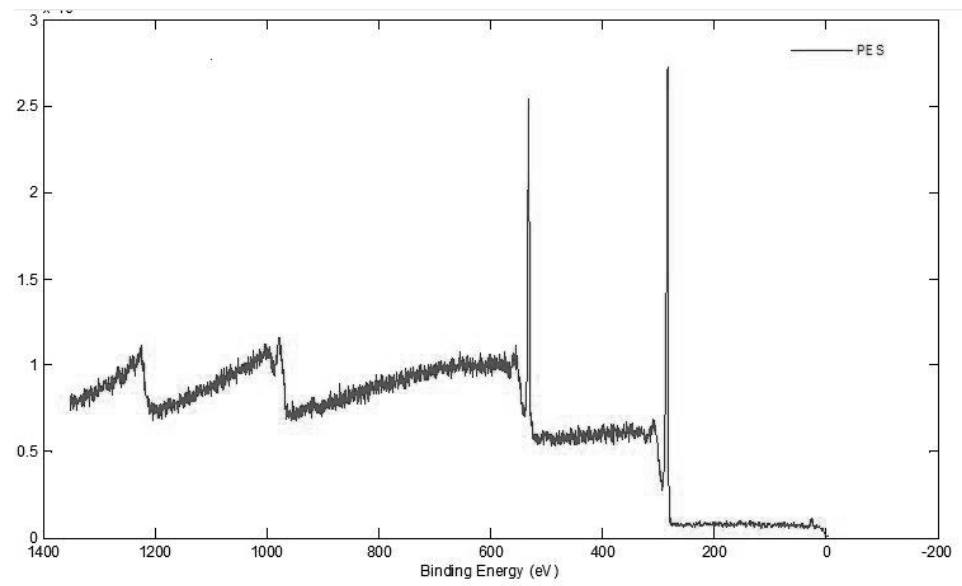

Figure 8.8a: XPS spectral scan of PES sample.

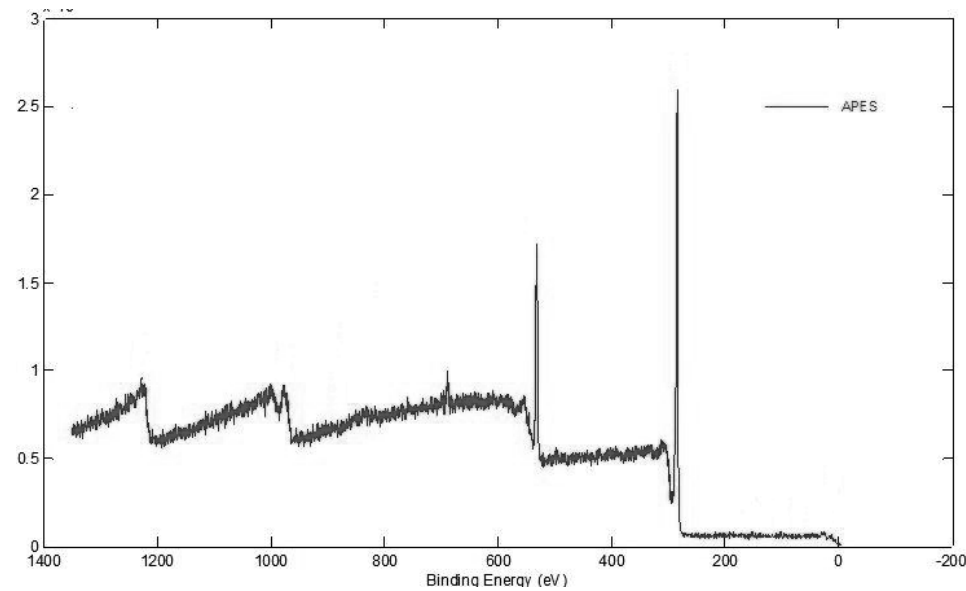

Figure 8.8b: XPS spectral scan of APES sample.

Figure $8.8 \mathrm{~b}$ shows the XPS spectral scan of APES samples. Here it is seen that the $\mathrm{C}_{1 \mathrm{~s}}$ peak is increased while the $\mathrm{O}_{1 \mathrm{~s}}$ peak is reduced as compared to the PES sample. This is in agreement with the findings in the literature [14]. 


\begin{tabular}{|c|c|c|c|c|c|c|}
\hline Sample & $\mathrm{O} / \mathrm{C}$ & $\begin{array}{c}\text { C-C-C } \\
284.8 \mathrm{eV}\end{array}$ & $\begin{array}{c}\mathrm{C}-\mathrm{C}-\mathrm{O} \\
286.3 \mathrm{eV}\end{array}$ & $\begin{array}{c}\mathrm{O}-\mathrm{C}=\mathrm{O} \\
288.8 \mathrm{eV}\end{array}$ & $\begin{array}{c}\mathrm{C}=\mathrm{O} \\
532.0 \mathrm{eV}\end{array}$ & $\begin{array}{c}\text { C-O-C } \\
533.4 \mathrm{eV}\end{array}$ \\
\hline PES & 0.33 & 65 & 25 & 10 & 55.5 & 44.4 \\
\hline APES & 0.25 & 73 & 18 & 9 & 50.9 & 49.1 \\
\hline
\end{tabular}

Table 8.1: Results of the XPS analysis of the PES and APES samples.

Table 8.1 shows the results of the XPS analysis done on both the samples in the form of the oxygen to carbon ratio and the relative amount of the different carbon and oxygen bonds on the samples. The oxygen to carbon ratio is reduced to 0.25 on the activated sample (APES) from 0.33 of the untreated PES. An increase in the C-C-C concentration at $284.8 \mathrm{eV}$ is observed in the APES sample. This is due to the increase in the carbon content (from the acrylic acid polymer) on the surface of APES. The literature describes that the decomposition of the $\mathrm{O}_{1 \mathrm{~s}}$ peak into peaks characteristic of $\mathrm{OH}$ groups, $\sigma$ and $\Pi$ bonded oxygen result in an increase in the $533.3 \mathrm{eV}$ peak. [3]. This was observed with the APES samples which lead to the conclusion that the activation step was successful.

\subsubsection{UV vis spectrophotometer measurements of $\mathrm{CHXB-BCD}$ and $\mathrm{CHXA-BCD}$ solutions}

UV vis spectrophotometer scans were done of solutions containing a mixture of the $\mathrm{BCD}$ and the respective guest molecules. As described earlier, the concentration of the guest molecule was kept constant at $30 \mu \mathrm{M}$ while that of BCD was increased steadily in these solutions. No shift in the CHXA/CHXB absorbance peak at $254 \mathrm{~nm}$ was observed in the guest-BCD solutions mixed with molar ratios of 1:1 to 1:40. The maximum absorbance peak of CHXA-BCD and CHXB-BCD solutions (as seen in Figures $8.9 \mathrm{a}$ and $8.9 \mathrm{~b}$ ) shifted from $254 \mathrm{~nm}$ to $258 \mathrm{~nm}$ only at and above BCD concentrations of $1.25 \mathrm{mM}$. These results indicated that a BCD molar excess of about 40 times to that of $\mathrm{CHXA} / \mathrm{CHXB}$ is needed for complexation to start in these experimental conditions. This implies that the complexation starts when there are 40 or more molecules of BCD in the solution for every CHXA and CHXB molecule. The absorbance values of the shifted peak increased with the increase in BCD concentrations in the mixed solutions. This is an indication of the increasing complexation between the BCD and the guest molecules as explained earlier. This means that (despite the total number of $\mathrm{CHXA} / \mathrm{CHXB}$ molecules added to the 
mixture remains constant) more of the guest molecules complex with the $\mathrm{BCD}$ molecules when the number of BCD molecules is increased in the guest-BCD mixture solutions.

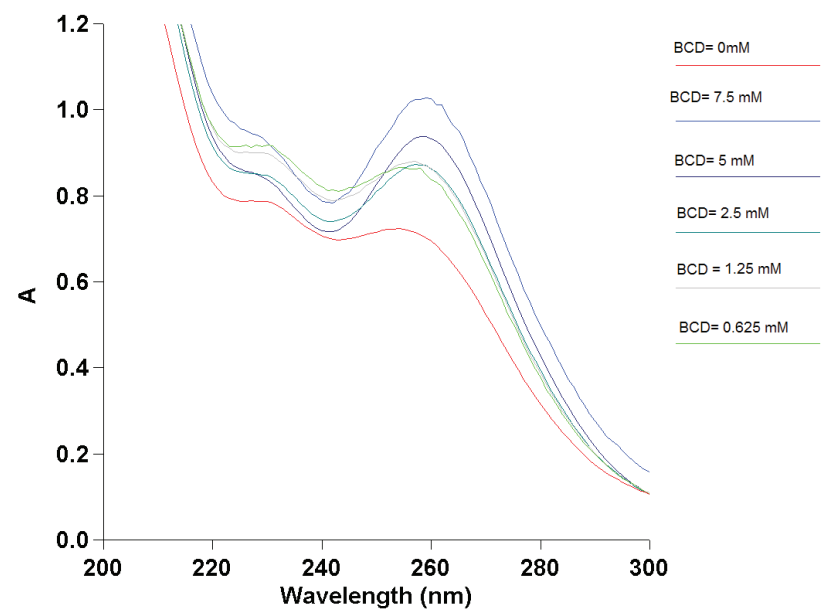

Figure 8.9a: The UV vis spectrophotometer absorbances (A) of $30 \mu \mathrm{M}$ of $\mathrm{CHXB}$ with varying concentrations of $\mathrm{BCD}$.

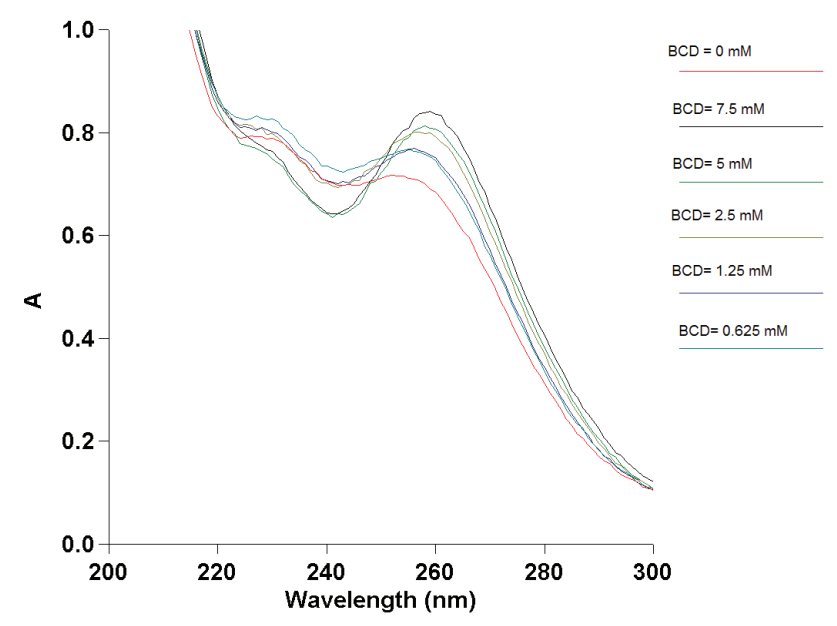

Figure 8.9b: The UV vis spectrophotometer absorbances (A) of $30 \mu \mathrm{M}$ of CHXA with varying concentrations of $B C D$.

The concentrations of BCD (in solution) required for the start of complexation with CHXA and then with $\mathrm{CHXB}$ was identical as can be in the above figures. The onset of complexation of the BCD-CHXA molecules was expected to occur at higher BCD concentrations (therefore at higher guest to $\mathrm{BCD}$ ratio) as compared to $\mathrm{BCD}-\mathrm{CHXB}$. 
This was due the reduced hydrophobicity and the higher molecular weight of CHXA in comparison with $\mathrm{CHXB}$. It was also hypothesized that this delay in the onset of complexation with CHXA would be observable in UV vis spectra. However in this spectral study except for the relative differences in absorbances, no differences were seen in the $\mathrm{BCD}$ concentrations required for the onset of complexation between these two types of chlorhexidine. The same set of experiments were repeated in presence of a $2.5 \mathrm{~cm}$ by $2.5 \mathrm{~cm}$ cotton fabric (in test tubes containing the BCD-guest solutions). As earlier, the shift in the maximum absorbance of $\mathrm{CHXA}$ and $\mathrm{CHXB}$ remained at a BCD concentration of $1.25 \mathrm{mM}$.

Chlorhexidine consists of two hydrophobic p-chlorophenol rings which are positioned at the end of the structure. The cationic hydrophilic biguanide groups lie adjacent to these aromatic rings and the hydrophobic hexamethylene groups lie in the center. The complexation of chlorhexidine with the BCD cavity involves the entry of only the hydrophobic phenyl group (which are also the chromophore moieties) into the BCD cavity (towards the narrower side of the cavity) [4]. The adjacent hydrophilic biguanide groups do not enter the cavity due to the apolar-polar repulsion. These polar groups on the other hand form hydrogen bonds with the secondary hydroxyl groups at the rim of the BCD molecule. In case of CHXA, the diacetate group does not enter the cavity either. Therefore it was initially concluded that the higher water solubility (or the increased molecular weight) of CHXA due to the diacetate group does not influence the onset of complexation in any way due to identical and partial complexation of the apolar groups into the BCD cavity in either form of the guest molecules.

However, on closer observation of the pharmaceutical industry protocol it appeared that this conclusion was incorrect. The industry procedure follows the addition of solid CHXA/CHXB to aqueous BCD solution. In such cases, complexation is reported to start with a BCD molar excess of 0.5 to 0.75 times [15]. In this work, $\mathrm{CHXA} / \mathrm{CHXB}$ were dissolved in water prior to the addition to BCD solution. A concentration of $30 \mu \mathrm{M}$ was low enough for both the forms (especially $\mathrm{CHXB}$ ) to dissolve in water easily. Therefore such a situation is not conducive for complexation with BCD where the guest molecules are already solubilized in water. This is the due to the fact that complexation would require the breaking of the already established 
hydrogen bonds between $\mathrm{CHXA} / \mathrm{CHXB}$ and water in favour of hydrophobic interactions of $\mathrm{CHXA} / \mathrm{CHXB}$ with the BCD cavity.

It is known in the literature that presence of BCD has minimal influence on the solubility of already soluble drugs [16]. In case the $\mathrm{CHXA} / \mathrm{CHXB}$ were added directly to BCD solutions in excess of their solubility limit, a complexation with BCD would start immediately i.e at lower guest to BCD mixture ratios. This would due to the simultaneously available hydrophobic interactions (for the apolar moieties of the guest molecules) and hydrogen bonds from water (for the polar groups of the guest molecules). In such a situation the onset of complexation will be significantly influenced by aqueous solubility limits of the two forms of chlorhexidine.

Further no new differences (with regard to the shift in the peak) were observed in spectra obtained during the complexation of BCD with the two guest molecules in the presence of cotton fabrics (not shown here). This implied that the electrostatic interaction of $\mathrm{CHXA} / \mathrm{CHXB}$ with cotton and their complexation with the BCD molecules are not influenced by each other in the current experiments conditions.

The solubility or polarity of the guest molecules is known to also influence the stability of a complex [17] as well as the release of the guest molecule from the complex [18]. It is not known if this is applicable to the complexes of CHXA and $\mathrm{CHXB}$ which are only partially included into the BCD cavities. Determination of the binding rate constants of both $\mathrm{CHXA}$ and $\mathrm{CHXB}$ with $\mathrm{BCD}$ and the study of release of these molecules from BCD needs to be done in order to conclude further.

\subsubsection{Adsorption of CHXA on to cotton, BCD-cotton, APES and BCD-APES fabrics}

Literature reports that attachment of chlorhexidine to cotton is via electrostatic interactions. This is between the two cationic biguanide groups of chlorhexidine and the carboxylate groups of cotton. As with PHMB (described in chapter 3 and 5) at higher bulk concentrations a combination of electrostatic interactions and hydrogen bonding is known to exist between cotton and chlorhexidine. Computer models have verified this hydrogen bonding to be existing between the hydroxyl groups of cotton and the p-chlorophenol and the biguanide groups of chlorhexidine [5, 6]. 
The binding of CHXA to cotton is dependent on the number of carboxylate groups on cotton at lower bulk concentrations and on additionally the number of hydroxyl groups on the substrate at higher bulk concentrations. The binding of CHXA to BCDcotton is dependent on the number of carboxylate groups and the hydrophobic interactions of CHXA with BCD molecules (at the lower CHXA bulk concentrations) [19] and additionally with the available hydroxyl groups of BCD-cotton at higher bulk concentrations. The number of the free hydroxyl groups in BCD-cotton are expected to be much lower than in case of cotton due to some of them being used up in the esterification reaction with BTCA (in case of cotton).

The adsorption data of CHXA fixation on BCD-cotton and cotton is shown in Figure 8.10. It appears that up to a bulk concentration of $1 \mathrm{~g} / \mathrm{l}$, no significant difference is seen between the CHXA adsorption on BCD-cotton and cotton (control). The CHXA adsorption on BCD-cotton steadily increases thereafter in comparison with cotton. This increase in adsorption is by about a factor of 1.5 times (CHXA bulk concentrations of 1-2.5 g/l). However the Table 8.2 shows the numerical values and the differences in adsorption of CHXA on to the BCD fixed samples at each bulk concentration.

From the difference in the mass of CHXA adsorbed on to BCD-cotton-CHXA samples in comparison with cotton-CHXA, the number of CHXA molecules adsorbed per gram of fabric could be calculated (by taking the Avogadro constant into consideration). If a 1:1 complexation ratio between the BCD molecule and CHXA molecule is considered, then the number of CHXA molecules would equal the number of BCD molecules. As stated earlier, the amount of BCD fixed (and number of BCD molecules) on to the sample was also known. The occupancy percentage of BCD molecules (percentage of the fixed BCD molecules on the fabric that are occupied by the guest molecules) therefore could be further calculated at each CHXA bulk concentration.

It was stated earlier that a complexation ratio between BCD and CHXA is 1:2 in homogenous solutions. This complexation ratio does not seem plausible when BCD molecules are immobilized on to the surface of the textile fibres. In such a case the fixed BCD molecules are limited in their movement, orientation and the resulting 
complexation with guest molecules (as compared to the free movement in a homogenous solution). Therefore a 1:1 complex was assumed for the calculations of the occupancy percentage and these values are shown in Table 8.3.

During the exhaust treatment of the BCD-cotton with CHXA, the CHXA molecule shows an affinity towards the carboxylate groups of cotton over the complexation with the BCD molecules. This is corroborated by the small increase in mass of CHXA adsorbed on to BCD-cotton as compared to the control i.e. cotton (shown in Table 8.2). This can be explained from the results described in section 8.2.3 where it was seen that the complexation of a guest molecule with BCD is not immediate when it is completely solubilized in the aqueous solvent because BCD has little influence in the case of highly soluble guests. In the same set of experiments, it was also observed that presence of cotton did not influence the onset of complexation of CHXA with $\mathrm{BCD}$ in any way. It was also concluded that complexation is not influenced by the presence of electrostatic interactions that CHXA and CHXB have with cotton. Here it can be similarly concluded that the electrostatic interactions between CHXA and cotton and the hydrophobic interactions between BCD and CHXA are parallel processes and they do not significantly interfere with each other in these experimental conditions.

After the saturation of the electrostatic bonds and hydrogen bonds (seen from the flattening of the curve after $1 \mathrm{~g} / 1$ on cotton at higher CHXA bulk molecules), the CHXA molecules continue to be solely adsorbed on to the BCD-cotton fabrics (i.e into the $\mathrm{BCD}$ cavities on the substrate) via the only prevailing forces present which are the hydrophobic interactions between CHXA and the BCD cavities. It can be observed that the CHXA adsorbed does not significantly increase with the CHXA bulk concentration (nor does the occupancy percentage) at this later stage. This is due to the limitation in the number of cavities available for further complexation. This can be seen in the high occupancy percentage of BCD molecules at these bulk concentrations. From Table 8.3, it appears over $80 \%$ of the BCD molecules fixed on the cotton fabric have been occupied by the CHXA molecules.

The two assumptions in this work are that the complexation ratio between the immobilized BCD molecule and the CHXA molecule is 1:1 (while in solutions it is 
1:2 or more). In reality, it may be a mixture (if a BCD to CHXA complexation ratio of 1:2 is considered, then the occupancy percentage would be double of the values present in Table 8.3). The second is that the calculations pertaining to the occupancy percentage of BCD cavities assumes that all of the excess CHXA molecules contributed by the presence of BCD molecules are complexed within the BCD cavity. It is possible that some of the guest molecules are between the various BCD cavities (called non-inclusion complexes) with in the BTCA network.

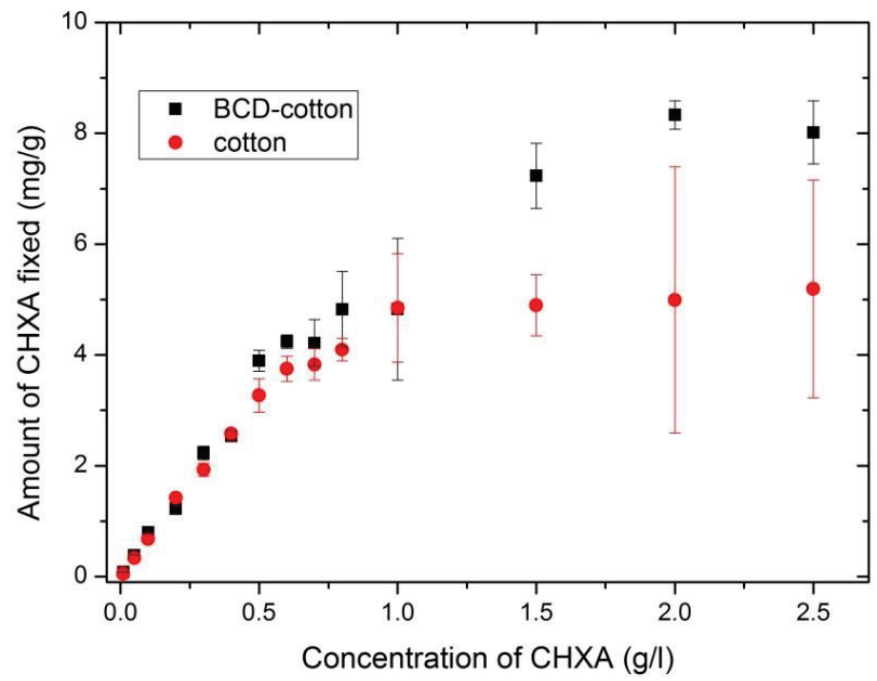

Figure 8.10: Adsorption values of CHXA in mg per gram of textile (cotton and BCD treated fabric) for the various bulk concentration of CHXA. 


\begin{tabular}{|c|c|c|c|c|}
\hline $\begin{array}{c}\text { CHXA bulk } \\
\text { concentration } \\
(\mathrm{g} / \mathrm{l})\end{array}$ & $\begin{array}{c}\text { CHXA fixed on } \\
\text { BCD-cotton (mg per } \\
\text { gram of fabric) }\end{array}$ & $\begin{array}{c}\text { CHXA fixed on } \\
\text { cotton }(\mathbf{m g} \text { per gram } \\
\text { of fabric })\end{array}$ & $\begin{array}{c}\text { Increase in mass } \\
\text { of CHXA } \\
\text { adsorbed in BCD- } \\
\text { cotton-CHXA } \\
\text { samples }(\mathbf{m g} \text { per } \\
\text { gram of fabric) }\end{array}$ & $\begin{array}{c}\text { The factor } \\
\text { increase in } \\
\text { CHXA }\end{array}$ \\
\hline 0.01 & 0.09 & 0.05 & 0.033 & $\begin{array}{c}\text { adsorption in } \\
\text { BCD-cotton- }\end{array}$ \\
\hline 0.05 & 0.39 & 0.34 & 0.051 & 1.62 \\
\hline 0.1 & 0.80 & 0.68 & 0.120 & 1.15 \\
\hline 0.2 & 1.23 & 1.42 & -0.194 & 1.18 \\
\hline 0.3 & 2.23 & 1.93 & 0.302 & 0.86 \\
\hline 0.4 & 2.54 & 2.58 & -0.043 & 1.16 \\
\hline 0.5 & 3.89 & 3.27 & 0.624 & 0.98 \\
\hline 0.6 & 4.24 & 3.75 & 0.491 & 1.19 \\
\hline 0.7 & 4.22 & 3.83 & 0.390 & 1.13 \\
\hline 0.8 & 4.83 & 4.10 & 0.729 & 1.10 \\
\hline 1 & 4.83 & 4.85 & -0.021 & 1.18 \\
\hline 1.5 & 7.23 & 4.90 & 2.335 & 1.00 \\
\hline 2 & 8.33 & 4.99 & 3.336 & 1.48 \\
\hline 2.5 & 8.01 & 5.19 & 2.822 & 1.67 \\
\hline
\end{tabular}

Table 8.2: Amount of CHXA fixed on BCD-cotton and cotton. 


\begin{tabular}{|c|c|c|}
\hline $\begin{array}{c}\text { CHXA bulk } \\
\text { concentration } \\
(\mathrm{g} / \mathrm{l})\end{array}$ & $\begin{array}{c}\text { Number of excess } \\
\text { CHXA molecules on } \\
\text { the BCD-cotton- } \\
\text { CHXA fabric }\end{array}$ & BCD occupancy \% \\
\hline 0.01 & $3.19 .10^{16}$ & 0.97 \\
\hline 0.05 & $4.90 .10^{16}$ & 1.48 \\
\hline 0.1 & $1.15 .10^{17}$ & 3.49 \\
\hline 0.2 & $-1.87 .10^{17}$ & -5.67 \\
\hline 0.3 & $2.90 .10^{17}$ & 8.80 \\
\hline 0.4 & $-4.12 .10^{16}$ & -1.25 \\
\hline 0.5 & $6.0 .10^{17}$ & 18.20 \\
\hline 0.6 & $4.72 .10^{17}$ & 14.33 \\
\hline 0.7 & $3.75 .10^{17}$ & 11.37 \\
\hline 0.8 & $7.01 .10^{17}$ & 21.26 \\
\hline 1 & $-2.02 .10^{16}$ & -0.61 \\
\hline 1.5 & $2.24 .10^{18}$ & 68.10 \\
\hline 2 & $3.21 .10^{18}$ & 97.30 \\
\hline 2.5 & $2.71 .10^{18}$ & 82.32 \\
\hline
\end{tabular}

Table 8.3: Occupancy percentage of BCD molecules fixed on BCD-cotton-CHXA fabric.

These adsorption experiments were done attempted with activated polyester, however, the experiments were not successful since it was found that the benzophenone/acrylic acid was leaching from the samples when placed in the CHXA exhaust liquor bath. The leaching of these components from samples interfered with the UV vis spectrophotometer absorbance measurements of CHXA in the bulk solution. Due to this the amount of CHXA fixed on the APES/BCD-APES fabric could not be calculated.

\subsubsection{Antibacterial experiments of CHXA treated fabrics}

The antibacterial experiments were done on cotton-CHXA, BCD-cotton-CHXA, APES-CHXA and BCD-APES-CHXA fabrics. All the fabrics were treated with CHXA bulk concentrations of $0.1-1 \mathrm{~g} / 1$. A range of antibacterial activity could be seen only between these application concentrations.

The results of the antibacterial tests are presented in Figure 8.11a and Figure 8.11b. The figures here show the amount of alive bacteria (CFUs) eluted from the fabrics 
after the required incubation time period. As expected there is a decrease in the log CFUs with the increasing CHXA bulk concentration. From Table 8.2, it is clear that amount of fixation of CHXA on BCD-cotton-CHXA is increased by a small amount in most of the treatments up to $1 \mathrm{~g} / 1$ of CHXA bulk concentration. However, the number of alive bacteria extracted from BCD-cotton-CHXA fabrics is consistently higher than the number eluted from cotton-CHXA fabrics for treatments at the same bulk concentration.

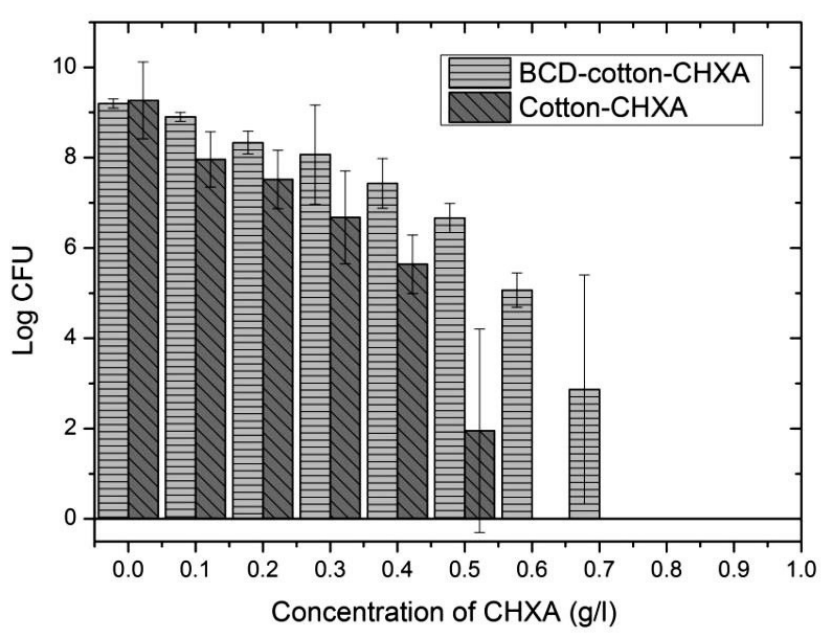

Figure 8.11a: Amount of bacteria eluted (shown in Log CFU) from cotton-BCD and control samples treated with various bulk concentrations of CHXA.

A similar trend was observed with the APES-CHXA and BCD-APES-CHXA samples. Figure $8.11 \mathrm{~b}$ shows that the amount of alive bacteria eluted from BCD-APES-CHXA samples is higher than the numbers eluted from APES-CHXA samples over the range of CHXA bulk treatment.

The antibacterial tests of the APES-CHXA and BCD-APES-CHXA samples treated above $0.5 \mathrm{~g} / 1$ of CHXA bulk concentration show values with high standard deviation. This is due to the limitation in the calculation method in the agar count plate testing method. 


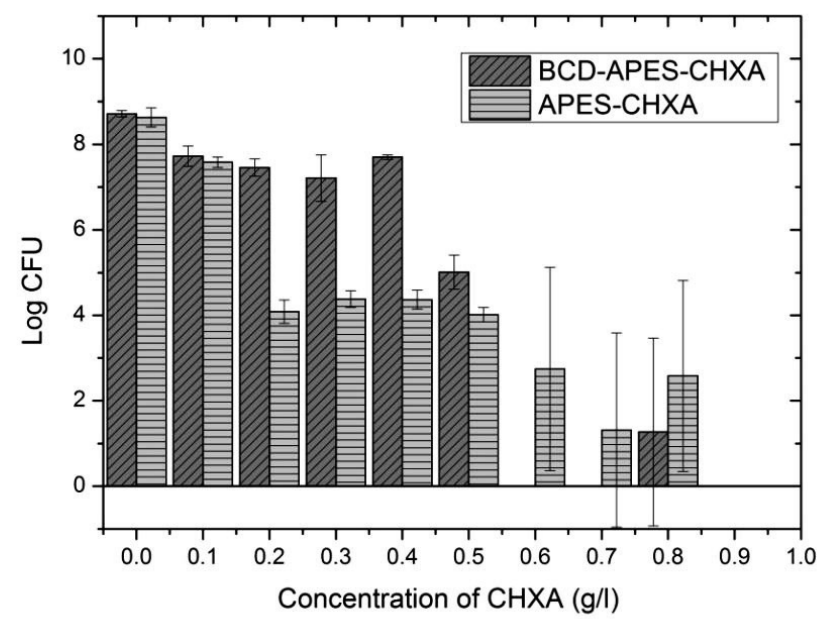

Figure 8.11b: Amount of bacteria eluted (shown in Log CFU) from APES-BCD and control samples treated with various bulk concentrations of CHXA.

This high standard deviation in the log CFU value of alive bacteria is seen in textile samples that harbor bacteria close to or below 6000 (CFUs). It was explained in chapter 3 that the agar plate count method can measure CFUs of only 6000 and above. Other examples of samples high standard deviation in the measured values are; at $0.5 \mathrm{~g} / \mathrm{l} \mathrm{CHXA} \mathrm{bulk} \mathrm{concentration} \mathrm{treatment} \mathrm{of} \mathrm{cotton-CHXA} \mathrm{and} 0.7 \mathrm{~g} / 1$ of bulk concentration treatment of BCD-cotton-CHXA in Figure 8.10a.

This difference in the antibacterial activity between the different treated samples is shown in Table 8.4. The table shows the AA values calculated according to Equation 2.2 of chapter 2 for the various CHXA bulk concentrations $(\mathrm{g} / \mathrm{l})$.

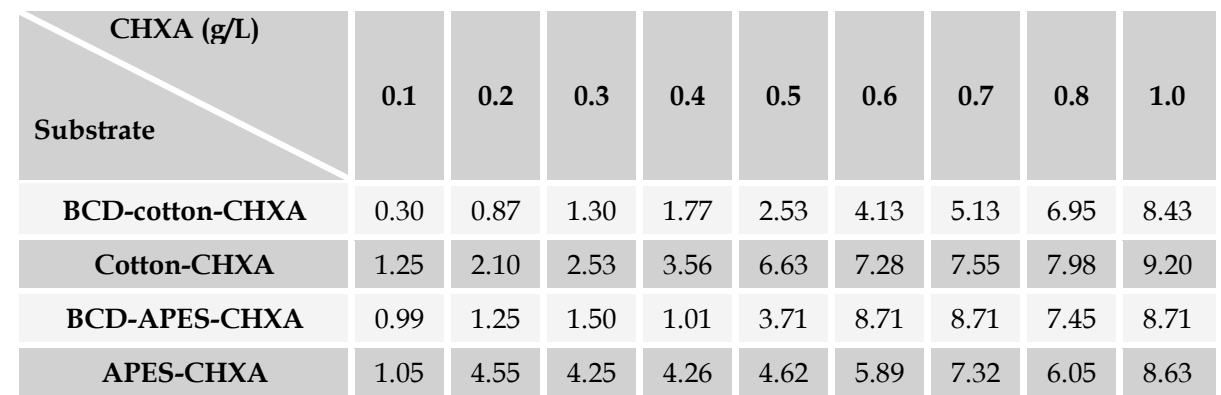

Table 8.4: The antibacterial activity of CHXA treated textiles. 
An AA of 2 is achieved at $0.2 \mathrm{~g} / 1$ of CHXA application for both cotton-CHXA and APES-CHXA samples. The same is achieved at $0.5 \mathrm{~g} / 1 \mathrm{CHXA}$ application concentration for BCD-cotton-CHXA samples and BCD-APES-CHXA samples. As explained in chapter 6 , this can be attributed to the slow release phenomenon of BCD molecule. Other studies have also reported this reduced antibacterial activity of BCD-antibacterial agent complexes [20-22]. The slow release phenomenon is known to be dependent on the binding constant between the guest molecule and the BCD molecule [23].

The slow release phenomenon of BCD treated cotton was proven with a simple CHXA release experiment. The experiment was done with samples treated with 1.5 $\mathrm{g} / 1$ of CHXA bulk concentration as explained in section 8.2.10. From Figure 8.10 it is already known that the amount of CHXA adsorbed by the BCD-cotton-CHXA sample is 1.5 times the cotton-CHXA sample at the same bulk concentration. As seen in Figure 8.12, the BCD-cotton-CHXA sample releases CHXA into water at a much lower rate than the cotton-CHXA sample. This lower rate of release of CHXA from the BCD-cotton samples supports the theory that the lower antibacterial activity of the BCD-cotton-CHXA samples is due to the slow release of CHXA complexed within the BCD cavities.

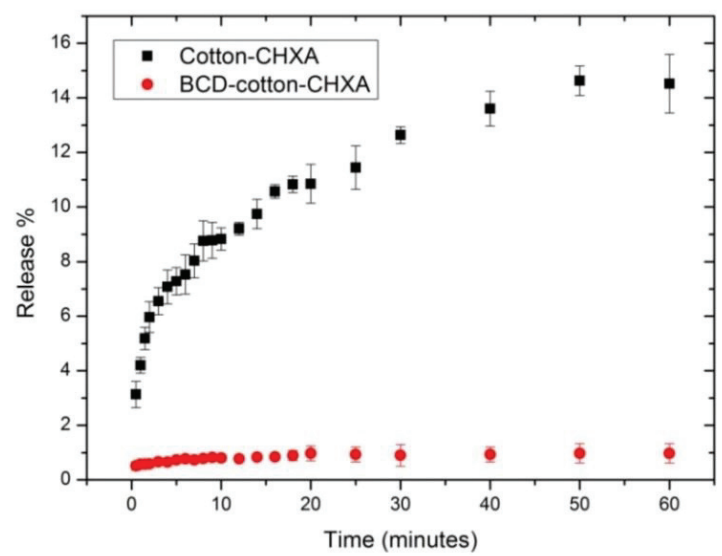

Figure 8.12: Release percentage of CHXA for Cotton-CHXA and BCD-cotton-CHXA sample. 
It is possible that the BTCA network with the BCD-cotton hinders the release of CHXA from the fabric to an extent. However, if this was the case the crosslinking would have inhibited or hindered the adsorption of the CHXA on to the BCD-cotton as well. The slow release phenomenon can further be confirmed by antibacterial tests of the homogenous BCD-CHXA solutions in comparison with CHXA solutions. However diffusion (of the agent from the fabric) plays a role in heterogeneous tests and this is absent in the homogenous tests.

This sustained or prolonged release of an antibacterial agent from the BCD cavity is advantageous in applications such as wound healing dressings. Optimal wound healing require dressings with sustained release of the antibacterial agent to prevent cell cytotoxicity and skin sensitivity issues (both of which are associated with quick release of antibacterial agents in such dressings) [24]. Such slow release also contributes to prolonging the shelf life of the fabrics. However, this slow release from the BCD cavities does not contribute in any way to the aims of the multi-step work where the host fixed fabrics are returned to the laundry within days of functionalization where they are to be treated again.

\subsection{Conclusion}

Unmodified $\beta$-cyclodextrin (BCD) was successfully attached to blends, polyester (PES) and activated polyester (APES). The fixation of BCDs to blends and PES was achieved via the crosslinker 1, 2, 3, 4 butane tetracarboxylic acid (BTCA). The activation of PES was done by the UV grafting of polyester with acrylic acid. XPS analysis of the APES and PES samples confirmed that the APES were successfully grafted with acid groups from the acrylic acid. The APES samples were then treated with BCD. The fixation was achieved via the hydrogen bonding between the hydroxyl groups of BCD and the acid groups on APES. Ester bonds were expected to form between the acid groups and the hydroxyl groups during the esterification reaction at the curing step. These two reactions were expected to not result in a high fixation amount and therefore BTCA was also added into the liquor to increase the solubility of BCD to more than five times its original solubility limit.

The amount of BCD fixed on the various samples was determined from the phenolphthalein method. APES samples showed the highest amount of BCD fixed as 
compared to the other fabrics. As concluded earlier in chapter 7 , the amount of BTCA did not have a significant influence on the amount of BCD fixed on the different substrates.

Moisture content measurements were done to study the influence of the BCD fixation on the moisture properties of the fabrics. The only BTCA treated samples showed a reduction in moisture content as compared to the untreated samples. This was due to the conversion of the hydroxyl or acid groups (cotton/blends and APES respectively) into their respective COOR groups. After the treatment of the samples with BCD an increase in the moisture content was seen due to the presence of polar hydroxyl groups of $\mathrm{BCD}$ on the surface of the textile.

For the antibacterial functionalization of the textile, chlorhexidine base (CHXB) and chlorhexidine diacetate (CHXA) were considered as the guest molecules. UV vis spectrophotometric measurements were done of CHXB-BCD and CHXA-BCD mixed solutions to study the differences in the $\mathrm{BCD}$ concentrations required for the onset of complexation of the two guest molecules with BCD. The onset of complexation started only after the addition of a molar excess of BCD molecules of about 40 times to that of CHXA/CHXB molecules. The complexation pattern of CHXA with BCD appeared identical to that of $\mathrm{CHXB}$ with BCD despite the differences in their solubility and polarity. This was explained by the fact that the guest molecules were entirely solubilized in the water prior to the spectral study. Complexation of a guest molecule with BCD cavities is expected to be immediate when the guest is added to the solvent in excess of its solubility limit.

The CHXA molecule was chosen to functionalize cotton, BCD attached cotton (BCDcotton), APES and BCD attached APES (BCD-APES). This choice was made based on its higher water solubility which would allow the use of a wide range of bulk concentration treatments in aqueous media. The antibacterial activity tests were done on the treated fabrics against E coli bacteria according to the Japanese standard described in chapter 2. The BCD fabrics treated with CHXA showed lower antibacterial activity as compared to control fabrics (treated with CHXA) for the same bulk concentrations. This lower antibacterial activity was attributed to the slow 
release phenomenon observed in BCD guest complexes. This slow release does not contribute in any significant way to the overall aims of the multi-step work.

It can be concluded that multi-step functionalization of the textile is not feasible for industrial laundries. The foremost reason for this is the poor wash fastness of the BCD molecules on the textile substrate as shown in chapter 7 . This poor wash fastness of the BCD treated fabrics would mean that the industrial work wear would have to be attached to the host molecule after every wash (in addition to the guest molecule). Apart from this, the sustained or control release of the antibacterial molecule in multi-step method does not result in any added advantage as compared to the quick release of the functional molecules of the single step method where the treated fabrics are returned to the laundry within days to be functionalized again.

\section{References}

1. Witono, J.R.B., New Materials by Grafting Acrylic Acid Onto Cassava Starch. 2012: University Library Groningen.

2. Yang, C.Q. and X. Wang, Infrared spectroscopy studies of the cyclic anhydride as the intermediate for the ester crosslinking of cotton cellulose by polycarboxylic acids. II. Comparison of different polycarboxylic acids. Journal of Polymer Science Part A: Polymer Chemistry, 1996. 34(8): p. 1573-1580.

3. Song, Y.W., et al., Effect of grafting of acrylic acid onto PET film surfaces by UV irradiation on the adhesion of PSAs. Journal of adhesion science and technology, 2006. 20(12): p. 1357-1365.

4. Qi, H., T. Nishihata, and J.H. Rytting, Study of the interaction between $\beta$ cyclodextrin and chlorhexidine. Pharmaceutical research, 1994. 11(8): p. 12071210.

5. Giménez-Martín, E., et al., Adsorption of chlorhexidine onto cellulosic fibers. Cellulose, 2009. 16(3): p. 467-479.

6. Blackburn, R.S., et al., Sorption of chlorhexidine on cellulose: mechanism of binding and molecular recognition. The Journal of Physical Chemistry B, 2007. 111(30): p. $8775-8784$.

7. Gilbert, P. and L. Moore, Cationic antiseptics: diversity of action under a common epithet. Journal of applied microbiology, 2005. 99(4): p. 703-715.

8. Denadai, Â.M., et al., Supramolecular self-assembly of $\beta$-cyclodextrin: an effective carrier of the antimicrobial agent chlorhexidine. Carbohydrate research, 2007. 342(15): p. 2286-2296. 
9. Chauhan, N., Cyclodextrin complexes of antimicrobial agents. 1995, PhD thesis, University of Ashton in Birmingham.

10.

http:// www.sigmaaldrich.com/catalog/product/sigma/c6143?lang=en\&reg ion=NL. Last accessed date: 7-1-2015.

11. Senior, N., Some observations on the formulation and properties of chlorhexidine. J Soc Cosmet Chem, 1973. 24: p. 259-78.

12. JIS (Japanese industrial standard) L 1902 in Absorption method, in Japanese Industrial Standard community: Tokyo. 2002.

13. http://www.lgcstandards-atcc.org. LGC standards last accessed date: 12.01.2014.

14. Tomšič, B., et al., Sol-gel technology for functional finishing of PES fabric by stimuli-responsive microgel. Journal of sol-gel science and technology, 2012: p. $1-14$.

15. Andrew Robert Gallopo, D.M.L., EP0306455 A1, Cyclodextrin complexes of bisbiguanido hexane compounds. 1989.

16. Loftsson, T. and M.E. Brewster, Pharmaceutical applications of cyclodextrins. 1. Drug solubilization and stabilization. Journal of pharmaceutical sciences, 1996. 85(10): p. 1017-1025.

17. Li, S. and W.C. Purdy, Cyclodextrins and their applications in analytical chemistry. Chemical Reviews, 1992. 92(6): p. 1457-1470.

18. Loftsson, T. and D. Duchene, Cyclodextrins and their pharmaceutical applications. International journal of pharmaceutics, 2007. 329(1): p. 1-11.

19. Tabary, N., et al., Functionalization of PVDF membranes with carbohydrate derivates for the controlled delivery of chlorhexidin. Biomolecular engineering, 2007. 24(5): p. 472-476.

20. Cusola, O., et al., Cyclodextrin functionalization of several cellulosic substrates for prolonged release of antibacterial agents. Journal of applied polymer science, 2012.

21. Bajpai, M., P. Gupta, and S. Bajpai, Silver (I) ions loaded cyclodextrin-graftedcotton fabric with excellent antimicrobial property. Fibers and Polymers, 2010. 11(1): p. 8-13.

22. Montazer, M. and E.B. Mehr, Na-diclofenac $\beta$-cyclodextrin inclusion complex on cotton wound dressing. The Journal of The Textile Institute, 2010. 101(5): p. 373379 . 
23. Roquette, Technical sheet Kleptose Betacyclodextrins and hydroxypropyl betacyclodextrins.

24. Atiyeh, B.S., et al., Effect of silver on burn wound infection control and healing: review of the literature. burns, 2007. 33(2): p. 139-148. 
Chapter 9 Conclusions and outlook 
The aim of this research work was the application of an antibacterial functionality on to textile substrates. This functionalization of the textile substrates was to be realized during the laundering process in industrial laundries. Two alternative methods were chosen for the application of the chemical on to the textile; the single step method \& the multi-step method. Polyhexamethylene biguanide (PHMB) was selected as the antibacterial molecule for the single step method. The exhaust application experiments described in chapter 4 showed the minimum PHMB bulk concentration required to obtain an antibacterial textile with the stipulated antibacterial activity.

As envisaged at the start of the work, the application of PHMB was done during the rinsing step of a laundering cycle at the industrial laundry. From the results of the experimental work done in this $\mathrm{PhD}$, recommendations (in regard to treatment conditions and bulk concentrations) were given to the personnel at the laundry for the industrial scale experiments. In the laboratory experiments the calculations regarding the chemical concentrations in the bulk was based on the standard $\% \mathrm{v} / \mathrm{v}$ or $\% \mathrm{w} / \mathrm{v}$. In the laundry the calculations regarding the dosing of the amount of chemical is based on the weight of the textiles in the wash extractor. The bulk concentrations suggested in this work were then recalculated to the 'on the weight of textile\%' for these industrial scale experiments.

Cotton and blends work wear were treated with PHMB in a wash extractor based on the given set of parameters. After the treatment, the fabrics were tested for their antibacterial activity. The treatment of the blends work wear resulted in the fabrics achieving the minimum required antibacterial activity value. Cotton work wear however failed to achieve this pre-determined limit. It appeared that this was due to the reactive dye present on the blue dyed cotton work wear which prevented the uptake of the PHMB from the bulk liquor on to the fabric (due to the incompatibility between the dye and PHMB). The blends on the other hand were not dyed with these reactive dyes. The cotton work wear was then washed a few times to remove the weakly fixed reactive dye and was then again treated with the recommended PHMB bulk concentrations. It appeared that after this washing the fabrics showed a significant increase in the PHMB uptake in the subsequent PHMB application. This was spectrophotometrically measured using the method described in chapter 5 . It is 
predicted that these pre-washed and subsequently treated fabrics will also show an improvement in the antibacterial activity as compared to the unwashed samples.

This PhD work also contains a mathematical model describing the textile adsorption and desorption kinetics of PHMB (chapter 5). The experimental work done in order to validate the model showed that the model described the adsorption kinetics for cotton and blends quite well. The model however could not be used to describe the PHMB adsorption kinetics on polyester substrate due to mathematical reasons. The model was also used to conclude that the initial moisture conditions of the fabric does not play a significant role in the adsorption kinetics of the PHMB molecule on to the various textile substrates. This meant that wet state of the fabric (in contrast to a dry fabric in the laboratory experiments) during the laundering process would not influence the kinetics of the PHMB uptake in a significant way. The successful functionalization of the blends work wear in the rinsing step of the laundering cycle supports this conclusion.

For the multi-step method, the native or unmodified $\beta$-cyclodextrin (BCD) was selected as the host molecule. The BCD molecule was attached to cotton substrate via the crosslinker, 1, 2, 3, 4, butane tetracarboxylic acid (BTCA) in an esterification reaction. The FTIR-ATR analysis of the $\mathrm{BCD}$ fixed cotton fabrics showed that the amount of ester formed on the fabric reduced after the treatment with BCD as compared to fabrics treated with only BTCA. This reduction in the ester bonds signified a reduction in the number of crosslinks within the textile. This was attributed to the steric hindrance of the BCD molecule on the esterification reaction. The reduced number of covalent ester linkages within the fabric (via BTCA) was considered to be one of the reasons behind the poor laundering durability of BCD on the textile. This poor wash durability BCDs which is in reality the poor wash durability of the ester bonds (linking BCD to the cotton) could also have to do with the low amount of the phosphate based catalyst used in this work. Further experimental work is needed to resolve the degree of influence that the catalyst concentration plays against the steric hindrance of the BCD molecule (on the esterification reaction and the subsequent crosslinking) on the wash durability of BCDs. This work also suggests that perhaps another crosslinker might be used instead of BTCA to circumvent these issues. The synthesis of new reactive BCD 
derivatives meant for textile fixation would also be another interesting line of research.

In this work $\mathrm{BCD}$ has been used only as a reservoir molecule to hold the guest molecules. However, its primary use lies in its ability to solubilize poorly soluble drugs and this was not explored in this work (since we wanted to study the influence of the guest bulk concentration on the antibacterial activity of the treated textiles). In pharmaceutical industry, poorly soluble drugs are added above their solubility limit to $\mathrm{BCD}$ solutions to solubilize amounts much higher than what is possible in the free form. This can done in the case of immobilized BCDs such as BCD fixed textiles by developing an exhaust method where a poorly soluble guest is continuously added to the bulk liquor and solubilized while simultaneously treating the BCD fixed textile with the guest. In effect it would result in adding an excess of the guest into the bath in comparison to the conventional method where the amount of chemical added is well below its solubility limit. This would not only result in the host fabric carrying much more of the guest molecules in comparison to the conventional method (i.e. increased BCD occupancy \% by the guest molecules) but this would also make the solubility limits of the guest molecule less relevant.

Chlorhexidine diacetate was chosen as the antibacterial molecule for the multi-step method. Host fixed fabrics (BCD-textile) and their controls were treated with CHXA in an exhaust bath. Antibacterial tests were done on these fabrics (BCD-textile-CHXA and textile-CHXA). The results of these tests showed that BCD-textile-CHXA samples showed lower antibacterial activity as compared to the control samples for the same bulk concentration treatments. This was despite a higher CHXA adsorption on to the host fixed fabrics due to presence of the BCD cavities. This lower antibacterial activity was attributed to the sustained or slow release phenomenon associated with BCD complexes. The sustained release of the BCD guest does not contribute in any way to the overall aim of this work. However, this has an advantage in other applications such as wound dressings and this is worthy of further investigation. Another disadvantage in the multi-step method was the poor wash durability of the BCDs which meant that the additional fixation of the host molecule was required prior to the application of the guest molecule. It therefore 
appeared that the multi-step method was not feasible for application in the industrial laundry. 
Summary 
The aim of this work was the antibacterial functionalization of textiles and its application in professional laundries. The antibacterial functionalization was meant for the various textile packages lent out by the laundry companies to their customers from hotels, hospital or food industries. Two distinct approaches were proposed for the functionalization step which was meant to be realized in the rinsing step of the laundering wash cycle. The first approach was referred to as the single step method where the antibacterial molecules are applied directly to the textile by the treatment in a bulk liquor (also referred to as the treatment bath method or the exhaust method). The second process referred to as the multi-step method involved two separate steps; the first being the application of a wash durable host molecule and the second being the application of the antibacterial molecule from the bulk liquor.

Chapter 1 describes the state of the art literature in antibacterial textile finishing. This chapter covers the various types of commonly found bacteria on textiles, the biodegradation of textiles by bacteria, the various types of antibacterial molecules used in antibacterial textile finishing and the classification of these antibacterial molecules based on their structure. This chapter briefly covers the concerns and risks to the humans with the use of such chemicals in the finishing treatments.

Chapter 2 describes the various antibacterial testing standards and the testing methods currently available and frequently used by the industry. Two commercial antibacterial textiles samples were procured and tested for their antibacterial activity in order to illustrate the differences in regard to the expression of the results between the various commonly used standards. Based on this review of the testing methods and the various standards, an antibacterial testing standard (JISL 1902 standard) and a testing method (the absorption method) was selected which was to be used in this work.

A time survivor study of bacteria is commonly done with various disinfectants to study the influence of the disinfectant concentration on the killing of bacteria. Polyhexamethylene biguanide (PHMB) was selected as the antibacterial molecule for the single step method. A time survivor study was then conducted on textiles treated with this agent against the E coli bacteria. This time survivor study of the PHMB treated textile is shown in chapter 3 . The results indicated that the fabrics treated 
with the lower PHMB bulk concentrations have dynamic antibacterial activity values in regard to the various incubation times. After an initial fall in numbers a regrowth of bacteria is observed at higher incubation times with these fabrics. The chapter also shows that Eosin staining of the PHMB treated fabrics can indicate or discern the presence of PHMB on the treated textiles from the untreated fabrics. The Eosin staining method however cannot be used for determination of the correlation between the PHMB bulk concentration treatments and colour strength of the stained fabric.

Chapter 4 shows the experimental work done to determine the minimum bulk concentration of the PHMB required to obtain textiles with the pre-determined antibacterial activity value. These experiments were done on cotton, blends and polyester. The antibacterial test results show that the treated fabric show a better antibacterial activity against Klebsiella pneumoniae as compared to Staphylococcus aureus. This chapter further also shows that the PHMB on the treated textiles is not durable to washing and a significant amount of the agent is removed from the surface of the fabric after the washing step. This poor wash durability has been attributed to the use of anionic detergents in the washing process due to which the cationic polyhexamethylene biguanide is deactivated.

A mathematical model was developed to describe the textile adsorption and desorption kinetics of the PHMB molecules. A general equation was derived taking into consideration the fabric properties, bulk volume, bulk concentration and the adsorption/desorption rate constants. Experimental work was then done to study the adsorption and desorption of the PHMB molecules from and to the textile substrate. This was done by measuring the concentration of the agent in the bulk using a US vis spectrophotometer. Experimental work showed that the treated fabrics showed no desorption of the agent into the bulk liquor. The general equation was then modified to exclude the influence of the desorption of the molecule and the adsorption rate constants were determined for the various substrates. These experiments were then further done for two different types of initial fabric moisture conditions; wet to wet and dry to wet. The wet to wet scenario was representative of the functionalization of the fabric in the rinsing step in the laundry while dry to wet scenario was representative of the fabric treatment done in the laboratory. The 
adsorption rate constants of the agent on to the fabric treated in wet to wet state appeared to be close to the rate constants determined in dry to wet conditions. These rate constants were then used to recalculate the model surface concentration of the agent on the fabric surface. The model appeared to describe the adsorption kinetics of the agent on to cotton and blends reasonably well. However, the model could not be used to describe the adsorption kinetics of the agent on to the polyester substrates.

The chapters 6-8 cover the multi-step work. Chapter 6 starts with overview of the basic structure of $\beta$-cyclodextrins, their chemical properties, uses and their fixation to textiles. This chapter describes the requirements of the guest molecules in order for a complex to be formed between the $\beta$-cyclodextrin cavity and the guest molecule. This chapter concludes with the recommendations for the three different types of $\beta$ cyclodextrins that can be used for fixation to textiles in the multi-step work. It is also concluded that due to the high water solubility and the high molecular weight of the PHMB molecule, it cannot be used as a guest molecule for the multi-step work. A new guest molecule for the same, (chlorhexidine diacetate) is recommended.

Chapter 7 describe the results of the experiments done with cotton substrate treated with the three types of $\beta$-cyclodextrins; the native or unmodified $\beta$-cyclodextrin, monochlorotriazinyl $\beta$-cyclodextrin and 2-hydroxy propyl $\beta$-cyclodextrin. The quantification of the amount of $\beta$-cyclodextrin fixed on to the substrate was done using the phenolphthalein method. The fixation results showed that cotton treated with the unmodified $\beta$-cyclodextrin (BCD) showed the highest amount of fixation as compared to the substrate treated with the other two types of $\beta$-cyclodextrins. BCD was fixed to cotton with the crosslinker 1,2,3,4 butane tetracarboxylic acid (BTCA) via the esterification reaction. This esterification reaction was between the hydroxyl groups of cotton/BCD and the acid groups of the crosslinker. FTIR-ATR scans were done on the fabrics treated with the various concentration of BTCA and BCD and compared with the control fabrics (treated with only BTCA). These results indicated that the amount of ester formed with in the cotton substrate reduced with the increase in BCD bulk concentration showing that the esterification reaction was impeded due to the presence of the (steric effects) $\beta$-cyclodextrin molecule. This also partially explained the poor wash durability of the BCDs fixed on to the cotton via the ester linkages. The other reason for the poor wash durability of the BCD molecule 
on the textile was considered to be due to the low amount of catalyst used in this work.

Chapter 8 elucidates the activation of polyester substrate with acrylic acid and its subsequent fixation with BCD. The activation of polyester was required due the lack of any reactive groups on its surface. The BCD fixed cotton and BCD fixed polyester were then respectively treated with chlorhexidine diacetate (CHXA). The adsorption of CHXA molecule on to the cotton substrate at various CHXA bulk concentration treatments was determined using a UV vis spectrophotometer. The results showed that the BCD fixed cotton had adsorbed higher amounts of CHXA as compared to the control sample. UV vis spectrophotometric studies were conducted on homogenous mixture solutions containing CHXA and BCD and these results were compared with mixture solutions of chlorhexidine base $(\mathrm{CHXB}$, which has much lower water solubility than $\mathrm{CHXA}$ ) and $\mathrm{BCD}$. These results led to the conclusion that the complexation between the BCD cavity and a guest molecule is preferentially driven when the amount of the guest added is much above its solubility limits. This was not the case in this work. It was then concluded that the complexation of CHXA with the BCD cavity and electrostatic interaction of CHXA with the cotton fiber were not influenced significantly by each other. The antibacterial experiments were done with the CHXA-BCD-textiles and their controls. The results showed that CHXA-BCDtextiles showed lower antibacterial activity as compared to the control fabrics (CHXA-textile) despite the former fabrics containing higher amount of CHXA for the same bulk concentration treatments. This was attributed to the slow release phenomenon of the BCD-guest complexes. This was then confirmed with the release experiments done on CHXA-BCD-cotton and CHXA-cotton samples.

Chapter 9 ends with conclusions from the single step and the multi-step work. This chapter briefly describes the results obtained from the industrial experiments done after the knowledge transfer of the PHMB application conditions to the laundry. Blends work wear treated with PHMB achieved the required antibacterial activity value for the recommended application concentrations. This was however not the case for cotton work wear. This was due to the presence of reactive dyes on the cotton work wear which impeded the PHMB uptake by the fabric. This problem was overcome to a certain degree by washing the cotton work wear several times to 
remove any excess dye present on the fabric. This chapter then presents a few recommendations for future investigations and research in the area of the multi-step method. 
Samenvatting 
Het doel van dit onderzoek is de antibacteriële behandeling van textiel en toepassing in industriële wasserijen. De behandeling vindt plaats tijdens het afspoelen van het wasgoed in het wasproces. Twee benaderingen worden gepresenteerd: een één-stap methode en een multi-stap methode. In het één-stap proces wordt het textiel direct toegevoegd in een behandelbad. Dit behandelbad bevat antibacteriële chemicaliën. De multi-stap methode bevat twee aparte stappen. In de eerste stap wordt een gastheer molecuul chemisch vastgezet op het textiel. In de tweede stap worden antibacteriële chemicaliën aangebracht.

Hoofdstuk 1 beschrijft de literatuurstudie naar antibacteriële textielbehandeling. In Hoofdstuk 2 worden antibacteriële teststandaarden en -methoden beschreven. $\mathrm{Na}$ analyse is de JISL 1902 standaard geselecteerd voor deze studie.

Polyhexamethyleenbiguanide ( PHMB ) werd geselecteerd als antibacterieel molecuul voor de één-stap methode. De antibacteriële testen werden uitgevoerd op katoen bij verschillende incubatietijden (0-24 uur). Uit de resultaten (beschreven in Hoofdstuk 3) blijkt, dat de antibacteriële werking dynamisch verandert bij verschillende incubatietijden (met name voor lagere bulkconcentraties PHMB op katoentextiel) .

In Hoofdstuk 4 wordt het experimentele werk beschreven, om de minimale bulkconcentratie van PHMB te bepalen die vereist is om textiel te verkrijgen met een vooraf bepaalde waarde van antibacteriële activiteit. Het textiel werd behandeld met verschillende bulkconcentraties PHMB. Deze experimenten werden uitgevoerd op katoen, katoen-polyester en polyester. De antibacteriële testresultaten laten zien dat het behandelde textiel een betere antibacteriële werking hebben tegen Klebsiella pneumoniae in vergelijking met Staphylococcus aureus. Dit hoofdstuk laat verder zien, dat de PHMB op het textiel niet duurzaam is tijdens het wassen. Na de wasstap wordt een aanzienlijke hoeveelheid van het middel verwijderd van het textieloppervlak. PHMB werkt slecht vanwege het gebruik van anionogene detergentia in het wasproces, waardoor de kationische polyhexamethyleenbiguanide wordt gedeactiveerd. 
Een wiskundig model werd ontwikkeld om de textiel adsorptie- en desorptiekinetiek van de PHMB moleculen te beschrijven. Dit model werd gevalideerd met experimenteel werk. Desorptie experimenten toonden aan dat de behandelde textiel geen desorptie van de PHMB naar de bulk laat zien. De algemene vergelijking werd vervolgens gewijzigd om de invloed van de desorptie van het molecuul na te gaan. De adsorptie snelheidsconstanten werden bepaald voor de verschillende substraten. Deze experimenten werden uitgevoerd op twee verschillende typen initiële textiel vochtcondities; nat naar nat (vertegenwoordiger van de behandeling in de wasserij) en droog naar nat (vertegenwoordiger van de textielbehandeling in het lab). De adsorptie snelheidsconstanten van PHMB in de nat naar nat toestand, bleek dicht bij de snelheidsconstanten van droog naar nat te liggen (met name voor katoenpolyester mengsels). Het model bleek de adsorptiekinetiek van de PHMB redelijk te beschrijven op katoen en katoen-polyester mengsels. Het model kon niet worden toegepast op polyester ondergronden.

De Hoofdstukken 6-8 beschrijven de multi-stap methode. Hoofdstuk 6 is een literatuuronderzoek over $\beta$-cyclodextrine, de chemische eigenschappen, toepassingen en de fixatie op textiel.

In Hoofdstuk 7 worden de resultaten van de $\beta$-cyclodextrine fixatie experimenten gepresenteerd, met katoen als substraat. Op basis van de resultaten van deze experimenten werd het ongemodificeerde $\beta$-cyclodextrine (BCD) gekozen als het gastheer molecuul. BCD werd bevestigd aan het katoen met de crosslinker 1,2,3,4butaan tetracarbonzuur (BTCA) via een veresteringsreactie. Deze veresteringsreactie vond plaats tussen de hydroxylgroepen van katoen / BCD en de zure groepen van de crosslinker. FTIR-ATR scans werden uitgevoerd op het textiel, behandeld met verschillende concentraties van BTCA en BCD en vergeleken met de referentie textiel (behandeld met alleen BTCA). De resultaten gaven aan dat de hoeveelheid ester gevormd met het katoensubstraat afnam met de toename van BCD bulkconcentratie. Het blijkt dat de veresteringsreactie werd belemmerd door de aanwezigheid van de $\beta$-cyclodextrine (sterische effecten). Dit verklaart deels de slechte BCD duurzaamheid tijdens het wassen. De andere reden voor de slechte duurzaamheid van BCD op het textiel, is de lage katalysator concentratie die werd gebruikt. 
Hoofdstuk 8 verduidelijkt de activering van het polyestersubstraat met acrylzuur en latere fixatie met BCD. De activering van polyester was nodig vanwege het gebrek aan reactieve groepen op het oppervlak. Het BCD-katoen en BCD-polyester werden vervolgens behandeld met chloorhexidine diacetaat (CHXA). De adsorptie van CHXA op het katoensubstraat bij verschillende CHXA bulkconcentratie behandelingen werd bepaald met behulp van een UV-Vis spectrofotometer. De resultaten lieten zien dat op de BCD-katoen grotere hoeveelheden CHXA was geadsorbeerd in vergelijking met de referenties. De antibacteriële experimenten werden gedaan met de CHXA-BCD-textiel en hun referenties. De resultaten vertoonden lagere antibacteriële activiteit van CHXA-BCD-textiel in vergelijking met de referentie-textiel (CHXA-katoen en CHXA-polyester), hoewel BCD behandelde textiel een grotere hoeveelheid CHXA bevat voor dezelfde bulkconcentratie behandeling. Dit kan worden toegeschreven aan de trage afgifte van het BCD-gast complex. Dit werd vervolgens bevestigd met de release experimenten, gedaan op CHXA-BCD-katoen- en CHXA-katoenmonsters.

Hoofdstuk 9 eindigt met de conclusies van het een-stap proces en het multi-stap proces. Dit hoofdstuk beschrijft de resultaten van de industriële experimenten. De kledingmix was behandeld met PHMB gedurende het wasproces. Hierdoor heeft de kleding de noodzakelijke antibacteriële activiteit niveau bereikt. Voor katoen was dit niet het geval, omdat er reactieve kleurstof aanwezig was. Om de overtollige kleurstof te verminderen, zijn extra wasstappen van de kleding nodig. Als afsluiting van dit hoofdstuk, worden een aantal aanbevelingen voor toekomstig onderzoek in het multi-stap proces gedaan. 
Acknowledgements 
This PhD work is a result of the support and contribution of great number of colleagues and friends. I consider this PhD not as a professional or career milestone but rather a personal one due to the fact that along this journey I had the privilege to meet and work with some of the most wonderful people, experience and understand the essence and the inner workings of life itself. I therefore take this opportunity to thank friends, well-wishers and colleagues.

My association with the EFSM group is of almost eight years. When I joined the group in 2007, I was quite inexperienced in the terms of knowledge and etiquette (scientific, professional, social, cultural, etc). I have the feeling that I did grow a great deal along the way. My foremost gratitude goes to my supervisor and promoter Marijn Warmoeskerken. He encourages independence, logical thinking and enquiry into the fundamentals of the subject. I am very grateful for his patience and persistence especially during the thesis writing stage. I am thankful to Marijn for all the support extended to me over the last many years including presenting me with innumerable opportunities to learn and grow, such as creating an opportunity to do a PhD when none was present to the final completion of the PhD (despite his retirement from the group). I would like to thank Heleen for the warm hospitality offered to me during the visits to Delft.

My second longest professional association has been with Henk Gooijer. I have learnt a good deal from Henk especially in regard to scientific methodology and spotting logical flaws in scientific reasoning. He has a vast array of know-how and a penchant for high speed thinking. He is always a phone call away in regard to any clarification and he has always extended a great deal of support to me right from my days as a technical assistant until now. Thank you, Henk.

My greatest gratitude extends to Dragan Jocic who has been a friend, colleague, and a fellow researcher through the years. I first approached Dragan for a position in his project in 2006. Though I did not get to work directly under him in the Advanbiotex project, he has been involved in my work through the years in various other capacities. Dragan has been a great support and a true well-wisher. He and Tatjana have always encouraged personal and professional growth and have been a dear friend to me and my family over the many years. 
I would like to extend thanks to my extraordinary friends from the group; Audrey, Paula, Veletia, Tatjana who consistently encouraged me and asked me not to give up when the going got tough. I have the greatest admiration for these women (\& mothers) who balance work and personal life so beautifully. They have most certainly been an inspiration.

Gerrit and Pramod have been very supportive of me in terms of encouragement and enthusiasm for which I am thankful. Both of them exemplify the meaning of the phrase "joie de vivre". Both are very intent on problem solving and supporting colleagues. Gerrit's advice "Be yourself" has stood me in good stead over time. Pramod was instrumental in bringing three students from Saxion who helped me with my experimental work described in this thesis apart from managing the project Wash \& Load which funded this PhD.

My biggest thanks to my bachelor students; Yvonne, Merlin and Jurjen. These three (along with Rosalinda) shared many an opinion over the Dutch way of life and habits. I learnt a great deal from these level leaded 20-something youngsters. I am thankful for their hard work, advice and humour. I probably learnt much more from them than they from me. I am grateful to Ton Lemmens (from Saxion) for his scientific support as well as for sending these students to our group.

I am very thankful to have met other wonderful people during the stay at the group; Cecile (ter Horst), secretary, colleague and friend rolled into one. She's a great listener and very empathetic. Thanks to the members of the ETE group who were gracious enough to adopt me into their group. Thank you Jacob, Nadia (especially both of you for the last minute favours I asked of you), Siti, Andre, Ernest and Wilma for their scientific help, fun, humour and good conversations. My thanks to other colleagues from UT and ex-colleagues from EFSM group who extended their kindness to me; Benno Knaken, Erna Luiten, Yvonne (Weber), Huib, Pelagia, Bertie, Eliza (Bottenberg), Majid, Hossein Barani, Tessa, Nils, Jolien, Brigita, Miroslav and members of the financial department (FEZ NH 256).

They were many people and friends from other groups and universities who supported me with tips in my microbiology work due to which I was able to carry on experimental work without taking time off for a course; Ron Hendrix, Mark Poels, 
Wendy Dankers, Miha Lavric, Hugo Alves, Kirsten Groener and Brigita Tomsic. My humble thanks to them.

I had also a great deal of communication with various companies who supplied us with many chemicals free of cost. I am thankful for their graciousness and patience in answering my long emails with scientific queries especially Maria Goret (Roquette) and Chris Chadwick (Lonza group).

There have been several kind people I met during my time in Germany and this is a good time to thank them (since this $\mathrm{PhD}$ is only a sojourn in the long journey). Sabine Albert and Prof. Manfred Gielhaupt (\& Angelika Gielhaupt), thank you for your kindness.

My deepest thanks and gratitude goes to my family; Chintan and Arav (for the great company during lab work on weekends, understanding, support, playfulness, humour and good cheer). Ma, Pa, Sup, Mammaya, and above all Amma (for it is only with her blessings that I could finish the $\mathrm{PhD}$ ). 


\section{About the Author}

Usha Rashmi Bhaskara was born on April 1'st, 1978 in Bangalore, India. After completing her high school studies from Clarence High School and Army School, Bangalore, India, she joined SKSJTI, Bangalore University for a Bachelors in Textile Technology (B.Tech) in the year 1996. In 2002, she joined the Master's program (M.Sc in Textile \& Clothing Management with specialization in Technology) in Niederrhein University of Applied Sciences, Moenchengladbach, Germany. From 2007-2010, she worked as technical assistant in the EFSM group where she worked on several short term scientific projects. In July 2010 she started work on her PhD, the result of which has been presented in this book. 
